\title{
PERIODIC ORBITS AND CHAIN-TRANSITIVE SETS OF $C^{1}$-DIFFEOMORPHISMS
}

\author{
by Sylvain CROVISIER
}

\begin{abstract}
We prove that the chain-transitive sets of $C^{1}$-generic diffeomorphisms are approximated in the Hausdorff topology by periodic orbits. This implies that the homoclinic classes are dense among the chain-recurrence classes.

This result is a consequence of a global connecting lemma, which allows to build by a $C^{1}$-perturbation an orbit connecting several prescribed points. One deduces a weak shadowing property satisfied by $C^{1}$-generic diffeomorphisms: any pseudo-orbit is approximated in the Hausdorff topology by a finite segment of a genuine orbit. As a consequence, we obtain a criterion for proving the tolerance stability conjecture in Diff ${ }^{1}(M)$.
\end{abstract}

CONTENTS

0. Introduction .

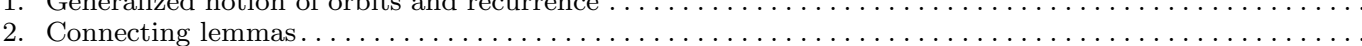

3. Generic properties of generalized orbits

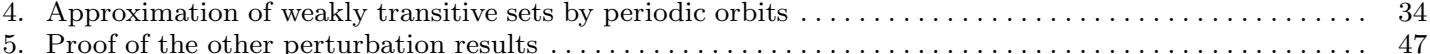

\section{Introduction}

\subsection{The shadowing lemma in hyperbolic dynamics}

In the study of differentiable dynamics, a remarkably successful theory, starting from the early sixties with Smale $\mathrm{Sm}_{2}$, describes a large class of systems: the uniformly hyperbolic systems. Their dynamics may exhibit complicated behavior but are well understood. For instance, Smale's spectral decomposition theorem asserts that the recurrent dynamics breaks down into finitely many invariant basic sets. Each of them is undecomposable (it contains a dense orbit), locally maximal (the only invariant subsets in a neighborhood are contained in the basic set itself) and contains a dense set of periodic points.

It also appears that these systems satisfy some stability properties, meaning that the orbit behavior does not change under small perturbations. Let $\operatorname{Diff}^{r}(M)$ be the space of $C^{r}$ diffeomorphism of a compact riemannian manifold $M$, endowed with the $C^{r}$-topology. A diffeomorphism $f \in \operatorname{Diff}^{r}(M)$ is called structurally stable in $\operatorname{Diff}^{r}(M)$ if any diffeomorphism $g \in \operatorname{Diff}^{r}(M)$ that is close to $f$ is conjugate to $f$ through a homeomorphism of $M$. Palis and Smale conjectured in $[\overline{P S}$ that the structurally stable diffeomorphisms 
in $\operatorname{Diff}^{r}(M)$ are the hyperbolic $C^{r}$-diffeomorphisms that satisfy the strong transversality condition: the stable and the unstable manifolds are transverse. Robin, de Melo and Robinson Rob, $\mathrm{D}, \mathrm{Rob}_{2}, \mathrm{Rob}_{3}$ have then proven that the hyperbolic diffeomorphisms that satisfy the strong transversality condition are structurally stable and also that the strong transversality condition is necessary for the structural stability. At the end of the eighties, Mañé $\mathbb{M}_{3}$ finished to prove the conjecture for the $C^{1}$-diffeomorphisms.

An important tool of the hyperbolic theory is provided by the shadowing lemma. Conley and Bowen have introduced $[\mathrm{B}, \mathrm{C}]$ the notion of $\varepsilon$-pseudoorbits of a dynamical system $f$. These are sequences of points $\left\{z_{n}\right\}$ in $M$ that generalize the orbits, since errors (of size smaller than $\varepsilon$ ) are allowed at each iterations. More precizely, for each $n$, the distance between $f\left(z_{n}\right)$ and $z_{n+1}$ is smaller than $\varepsilon$. Such a pseudo-orbit is $\delta$-shadowed by an orbit $\left\{f^{n}(x)\right\}$ if for any integer $n$, the points $z_{n}$ and $f^{n}(x)$ are at distance less than $\delta$. For any hyperbolic set $K$, the shadowing property is satisfied: for any scale $\delta>0$, there exists $\varepsilon>0$ such that to any $\varepsilon$-pseudo-orbit $\left\{z_{n}\right\}$ in $K$ one can associate a genuine orbit $\left\{f^{n}(x)\right\}$ which $\delta$-shadows the pseudo-orbit. Two important consequences of the shadowing lemma may be mentioned:

- If two periodic orbits in $K$ have points that are close, their stable and unstable manifolds intersect, implying that the two orbits are included in a same transitive set. This can be used to prove Smale's spectral theorem.

- One easily gets a form of stability of the hyperbolic systems, that is weaker than the structural stability. If the diffeomorphism $g$ is close enough to the map $f$, then, any orbit of $g$ that stays in a neighborhood of the hyperbolic set $K$ is shadowed by an orbit of the unperturbed system $f$. Let us now consider the non-wandering set $\Omega(f)$ of $f$, which supports, in some sense, the non-trivial dynamics. (A point is wandering if one of its neighborhoods is disjoint from all its iterates; $\Omega(f)$ is the set of points that are not wandering.) One says that $f$ is $\Omega$-stable in $\operatorname{Diff}^{r}(M)$ if for any diffeomorphism $g$ that is close to $f$ in $\operatorname{Diff}^{r}(M)$, the induced dynamics of $f$ and $g$ on $\Omega(f)$ and $\Omega(g)$ are conjugate by a homeomorphism. Using the shadowing property, one can prove that the hyperbolic systems whose basic sets don't have cycles (roughly speaking, this means that the basic sets are strictly ordered by the dynamics) are $\Omega$-stable in $\operatorname{Diff}^{r}(M)$. This result was originally proven by Smale in $\mathrm{Sm}_{2}$. Improving Mañé's result on the structural stability, Palis $\mathbb{P}]$ proved conversely that the hyperbolic systems that don't have cycles are the only $\Omega$-stable systems in $\operatorname{Diff}^{1}(M)$.

In parallel to these works, it appeared however that the hyperbolic diffeomorphisms fail to be dense in the set of differentiable systems. When the dimension of the manifold $M$ is equal or larger than 3, Abraham, Smale and 
Simon [AS, Si] have shown that $\operatorname{Diff}^{1}(M)$ contains non-empty open sets of non-hyperbolic dynamics. These examples are related to the existence of periodic orbits that have different indices (the dimension of their stable space) but contained in the same transitive set, producing some so-called heterodimensional cycles. Many generalizations of these examples were built in $\mathrm{Sh}_{2}$, $\mathrm{M}_{1}, \mathrm{BD}_{1}$. On surfaces, by looking at the homoclinic tangencies, Newhouse $\mathbb{\mathbb { N }}_{2}$, $\mathrm{N}_{3}$ discovered an other phenomena that produces open sets of non-hyperbolic diffeomorphisms. His result applies only in the $C^{r}$ topologies with $r \geq 2$. It was later generalized to manifolds of higher dimensions, see PV, Rom, GST.

\subsection{The generic dynamics}

In view of these results, it is also important to focus on the systems that are far from the hyperbolic dynamics. Some of them exhibit very degenerate phenomena (for instance, some coincide with the identity on an open set), but that can disappear by small perturbations of the dynamics. With the theory of generic dynamics, we forget these pathological systems, which represent a small part of the space of differentiable dynamics, and try to give a description of a large class of the remaining diffeomorphisms. In this paper we are interested by sets of diffeomorphisms that are residual for the Baire category (i.e. that contains a countable intersection of dense and open subsets of $\operatorname{Diff}^{r}(M)$ ). The results on the hyperbolic systems may indicate what kind of properties can be looked for: spectral decomposition, weaker forms of stability, shadowing properties, invariant splittings of the tangent bundle,... but new phenomena having some kind of persistence should also be introduced, in particular when tangencies or heterodimensional cycles occur: for example,

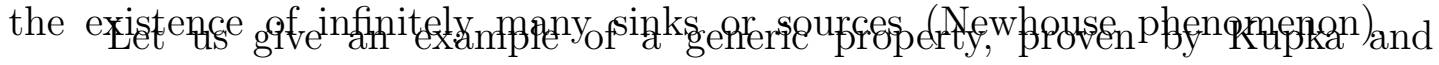
Smale [K], $\left[\mathrm{Sm}_{1}\right]$. For any diffeomorphism in a residual subset of $\operatorname{Diff}^{r}(M)$, the periodic orbits are all hyperbolic. Moreover their stable and unstable manifolds intersect transversally. Pugh then proved $\left[\mathrm{Pu}_{2}\right]$ that for $C^{1}$-generic diffeomorphisms, the periodic points are dense in the non-wandering set. It is a consequence of his closing lemma $\left[\mathrm{Pu}_{1}\right]$. By $C^{1}$-small perturbations, it is possible to create periodic points close to any non-wandering point. His techniques only work in the $C^{1}$ topology and explain why Mañé's theorem on the stability and most of the genericity results deal with the space $\operatorname{Diff}^{1}(M)$. Quite recently, Hayashi [H] improved this result and proved a connecting lemma that opened the door to many developments in $C^{1}$-generic dynamics. Let us consider two points $p, q$ whose orbits accumulate -one in the future and the other one in the past- on a same non-periodic point. Then, 
$p$ and $q$ can be connected by a segment of orbit for an arbitrarily small $C^{1}$-perturbation of the dynamics. Using these techniques, we were able, with Bonatti, to get a connecting lemma for pseudo-orbits BC.

Connecting lemma for pseudo-orbits. - Let us consider a diffeomorphism $f$ whose periodic orbits are hyperbolic. Let $x, x^{\prime}$ be two points that may be connected by $\varepsilon$-pseudo-orbits of $f$ for any constant $\varepsilon>0$.

Then, there exist arbitrarily small $C^{1}$-perturbations $g$ of $f$, such that $x$ and $x^{\prime}$ belong to the same orbit of $g$.

As for the structural and the $\Omega$-stabilities, the shadowing property is not generic. Bonatti, Díaz and Turcat have proven in [BDT] that there exists a non-empty open set of $C^{1}$-diffeomorphisms over a 3 -dimensional manifolds where the shadowing property failst. One of the main results of this paper however shows that a weaker property holds $C^{1}$-generically. The pseudo-orbits may be approximated by genuine orbits if one forgets the time parameterization and uses the Hausdorff topology. Let us recall that two compact sets $\mathcal{O}$ and $\mathcal{X}$ are $\delta$-close for the Hausdorff distance if the $\delta$-neighborhood of $\mathcal{X}$ contains $\mathcal{O}$, and the $\delta$-neighborhood of $\mathcal{O}$ contains $\mathcal{X}$.

Theorem 1. - There exists a residual subset $\mathcal{G}_{\text {shadow }}$ of $\operatorname{Diff}^{1}(M)$ such that any diffeomorphism $f \in \mathcal{G}_{\text {shadow }}$ satisfies the following weak shadowing property:

For any $\delta>0$, there is $\varepsilon>0$ such that to any $\varepsilon$-pseudo-orbit $\mathcal{X}=$ $\left\{z_{0}, z_{1}, \ldots, z_{n}\right\}$, one can associate a segment of orbit $\mathcal{O}=\left\{x, f(x), \ldots, f^{m}(x)\right\}$ which is $\delta$-close to $\mathcal{X}$ for the Hausdorff distance.

Moreover if the pseudo-orbit $\mathcal{X}$ is periodic (i.e. $z_{n}=z_{0}$ ), then, the point $x$ can be chosen m-periodic.

The weak shadowing property we get is different and stronger than the usual weak shadowing property introduced by Corless and Pilyugin in [CP] and studied by Sakai"]. Among other results, Sakai proves [Sa] that if $M$ is a surface, the interior of the set of the $C^{1}$-diffeomorphisms which have the weak shadowing property are the diffeomorphisms that satisfy the axiom A and the no-cycle condition. A more detailed exposition is given in $[\mathrm{Pi}$.

1 The dynamics of these diffeomorphisms is transitive and the non-wandering set is the whole manifold. Some generalizations of this result exist $[\mathrm{YY}, \mathrm{AD}]$. Hence, even in restriction to the non-wandering set, the shadowing property fails on these examples.

2 These authors require that for any $\varepsilon$-pseudo-orbit $\left\{z_{n}\right\}_{n \in \mathbf{Z}}$, there exists $x \in M$ such that $\left\{z_{n}\right\}_{n \in \mathbf{Z}}$ is contained in the $\delta$-neighborhood of the orbit $\left\{f^{n}(x)\right\}_{n \in \mathbf{Z}}$. Consequently, a transitive diffeomorphism has this weak shadowing property. 
In the proof of theorem 1, the connecting lemma for pseudo-orbits is needed but is not sufficient. It allows to show that for each pair of points $\left(z, z^{\prime}\right)$ in the pseudo-orbit $\mathcal{X}$, there is a segment of orbit $\mathcal{O}_{z, z^{\prime}}$ that intersects the balls centered at $z$ and $z^{\prime}$, with radius $\delta$; but the segment of orbit could be different for each pair $\left(z, z^{\prime}\right)$. In some sense, the connecting lemma for pseudo-orbits is semi-global. One connects two different points $z, z^{\prime} \in M$ by a perturbation which is global in $M$, but we don't have any control on the support of the orbit that joints $z$ to $z^{\prime}$. (In comparison, the closing lemma and Hayashi's connecting lemma are local results: the perturbations are local.)

For proving theorem 1, we need a new $C^{1}$-perturbation result which is global. In particular, we will discuss the following question:

Question. - Let $U_{1}, \ldots U_{k}$ be some (open) regions of the manifold $M$. Which assumptions would imply the existence of an orbit that crosses all these regions?

We first define the sets that may be approximated by orbits by perturbation. Let $\mathcal{X}$ be a compact set which is invariant by $f$. For any points $x, x^{\prime}$ in $\mathcal{X}$, we say that $x^{\prime}$ is a weak iterate of $x^{\prime}$ in $\mathcal{X}$ (this will be denoted by $\left.x \prec \mathcal{X} x^{\prime}\right)$ if for any non-empty open sets $U$ and $U^{\prime}$ that contain $x$ and $x^{\prime}$ respectively and for any neighborhood $W$ of $\mathcal{X}$, there exists a segment of orbit $\left\{z, f(z), \ldots, f^{n}(z)\right\}$ contained in $W$, such that $z$ belongs to $U, f^{n}(z)$ belongs to $U^{\prime}$ and $n$ is greater than or equal to 1 . The set $\mathcal{X}$ is a weak orbit of $f$ if any distinct points $x, x^{\prime}$ in $\mathcal{X}$ may be compared by the relation $\prec \mathcal{X}$. For example, the closure and the $\omega$-limit set of any orbit are weak orbits. Sometimes, one will consider weak orbits that are "dynamically ordered": one requires that $\prec_{\mathcal{X}}$ is transitive (so that it is a total ordering).

We will also need the following technical generic assumption:

(A) For any integer $n \geq 1$, the periodic points of $f$ of period $n$ are isolated in $M$.

This condition holds for instance when all the periodic orbits are hyperbolic. Kupka and Smale's theorem thus imply that (A) is generic in any space $\operatorname{Diff}^{r}(M)$ for $r \geq 1$.

The announced global perturbation result is the following:

Theorem 2. - Let $f$ be a diffeomorphism that satisfies condition (A), $\mathcal{U}$ a neighborhood of $f$ in $\operatorname{Diff}^{1}(M)$ and $\mathcal{X}$ a weak orbit of $f$ such that $\prec_{\mathcal{X}}$ is transitive. Then, for any $\eta_{0}>0$, there exist a diffeomorphism $g \in \mathcal{U}$ and a full orbit $\mathcal{O}(x)=\left\{g^{n}(x), n \in \mathbf{Z}\right\}$ of $g$ whose closure is $\eta_{0}$-close to $\mathcal{X}$ for the Hausdorff distance. 
Of course, the orbit $\mathcal{O}(x)$ we obtain in this theorem is in general not periodic. (For instance if the weak orbit $\mathcal{X}$ of $f$ contains a source $p$ and a sink $q$, then the orbit $\mathcal{O}$ of $g$ still accumulates on $p$ in the past and on $q$ in the future.) So, we have another version of the result which deals with weak orbits that have a recurrence property: we will say that a compact and invariant set $\mathcal{X}$ is weakly transitive iff for any non-empty open sets $U$ and $V$ that intersect $\mathcal{X}$ and any neighborhood $W$ of $\mathcal{X}$, there exists a segment of orbit $\left\{x, f(x), \ldots, f^{n}(x)\right\}$ contained in $W$ and such that $x$ belongs to $U$, $f^{n}(x)$ belongs to $V$ and $n$ is greater than or equal to 1 . (Note that by choosing $U=V$, one sees that $\mathcal{X}$ is contained in the non-wandering set $\Omega(f)$.) As an example, any transitive set is a weakly transitive set. Moreover any weakly transitive set $\mathcal{X}$ is also a weak orbit whose relation $\mathcal{X}$ is transitive.

Theorem 3. - Let $f$ be a diffeomorphism that satisfies condition (A), $\mathcal{U}$ a neighborhood of $f$ in $\operatorname{Diff}^{1}(M)$ and $\mathcal{X}$ a weakly transitive set of $f$. Then, for any $\eta_{0}>0$, there exist a diffeomorphism $g \in \mathcal{U}$ and a periodic orbit $\mathcal{O}$ of $g$ that is $\eta_{0}$-close to $\mathcal{X}$ for the Hausdorff distance.

In the next two sections we discuss the consequences of these theorems for the stability and the spectral decomposition of the $C^{1}$-generic diffeomorphisms. The reader will find other corollaries in section 3 .

\subsection{Tolerance stability}

An important goal of dynamical systems is to describe how the dynamical invariants change under perturbations. This leads to the notion of stability. Once people have discovered that the sets of structurally stable and $\Omega$-stable diffeomorphisms are not dense, they tried to find weaker forms of stability satisfied by a larger class of systems (see [Sh] $]$ ). At the beginning of the seventies, following an idea of Zeeman, Takens formulated [ [ $\left.\Gamma_{1}\right]$ an interesting notion of stability: the tolerance stability, which asserts that the orbit structure of a system varies only a little under small perturbations.

More precisely, for $r \geq 0$, we say that a diffeomorphism $f \in \operatorname{Diff}^{r}(M)$ of $M$ is tolerance stable in $\operatorname{Diff}^{r}(M)$ if for any $\varepsilon>0$, there exists a neighborhood $\mathcal{U}$ of $f$ in $\operatorname{Diff}^{r}(M)$ which satisfies the following: for any diffeomorphisms $g$ and $g^{\prime}$ in $\mathcal{U}$, and any orbit $O=\left\{g^{n}(x), n \in \mathbf{Z}\right\}$ of $g$, there exists an orbit $O^{\prime}=\left\{g^{\prime n}\left(x^{\prime}\right), n \in \mathbf{Z}\right\}$ of $g^{\prime}$ such that $O$ is contained in the $\varepsilon$-neighborhood of $O^{\prime}$ and $O^{\prime}$ is contained in the $\varepsilon$-neighborhood of $O$ (i.e. the closures of $O$ and $O^{\prime}$ are $\varepsilon$-close for the Hausdorff topology). This may be also defined in the

\footnotetext{
3 An equivalent definition is: for any $x, x^{\prime}$ in $\mathcal{X}$, the point $x^{\prime}$ is a weak iterate of $x$ in $\mathcal{X}$.
} 
following elegant way: recall that the set of non-empty compact subsets of $M$, endowed with the Hausdorff distance, is a compact metric space, that we denote by $\mathcal{K}(M)$. Hence, the closures of all the orbits of a diffeomorphism $f$ of $M$ gives a subset of $\mathcal{K}(M)$. Taking its closure in $\mathcal{K}(M)$, we obtain an element $\operatorname{Orb}(f)$ of $\mathcal{K}(\mathcal{K}(M))$. A diffeomorphism $f$ is tolerance stable in $\operatorname{Diff}^{r}(M)$ if it is a continuity point of the map $g \mapsto \operatorname{Orb}(g)$ from $\operatorname{Diff}^{r}(M)$ to $\mathcal{K}(\mathcal{K}(M))$. In [T] Takens stated the following conjecture'

Tolerance stability conjecture (Zeeman). - For any compact manifold $M$ and any $r \geq 0$, the tolerance stable diffeomorphisms are generic in $\operatorname{Diff}^{r}(M)$.

Since the orbits of a perturbed map $g$ close to a diffeomorphism $f$ are pseudo-orbits of $f$, it is very natural to look for a new formulation of the tolerance stability using pseudo-orbits. Takens defined in $\left[\Gamma_{2}\right]$ the notion of extended orbit, which is even more general than the weak orbits defined above: a compact set $\mathcal{X}$ which is invariant by $f$ is an extended orbit if for any $\varepsilon>0$ there exists an $\varepsilon$-pseudo-orbit contained in $\mathcal{X}$ and that is $\varepsilon$-dense in $\mathcal{X}$. The set of all the extended orbits is closed in $\mathcal{K}(M)$ and defines an element $\mathbf{E O r b}(f)$ of $\mathcal{K}(\mathcal{K}(M))$. If one replaces the orbits by the extended orbits in the definition of the tolerance stability, one gets the notion of extendedtolerance stability. (Equivalently, a diffeomorphism is extended-tolerance stable in $\operatorname{Diff}^{r}$ if it is a continuity point of the map $g \mapsto \operatorname{EOrb}(g)$.) Takens proved by semi-continuity arguments that the extended tolerance stable diffeomorphisms in $\operatorname{Diff}^{r}(M)$ are $C^{r}$-generic. Hence, he deduced the following criterion to get the genericity of the tolerance stability:

Criterion 1 (Takens). - If the set of diffeomorphisms $f$ such that $\operatorname{Orb}(f)=$ $\operatorname{EOrb}(f)$ is residual in $\operatorname{Diff}^{r}(M)$, then, the tolerance stability conjecture holds in $\operatorname{Diff}^{r}(M)$.

Takens proved in [T] some related results that motivated the conjecture. If $T$ is a map from $\operatorname{Diff}^{r}(M)$ to a topological space $X$, a diffeomorphism $f$ is $T$-tolerance stable in $\operatorname{Diff}^{r}(M)$ if it is a continuity point of $T$. For instance, $T$ may associate to any diffeomorphism $f$, the set $\mathbf{C l}(f)$ of invariant compact subsets of $M$, which is an element of $X=\mathcal{K}(\mathcal{K}(M))$. He showed that the Cl-tolerance stable diffeomorphisms are generic in $\operatorname{Diff}^{1}(M)$.

Another important example for $T$ is the map which associates the support of the non-trivial dynamics, viewed as an element of $\mathcal{K}(M)$. In general, there is no canonical definition of the non-trivial dynamics. It should at least contain all the periodic orbits: their closure will be denoted by $\mathcal{P} \operatorname{er}(f)$.

\footnotetext{
4 In the AMS review of the paper [Wh], Takens explains that Zeeman never published this conjecture.
} 
Another possibility for the non-trivial dynamics is the chain-recurrent set $\mathcal{R}(f)$ : it contains the points that belong to periodic $\varepsilon$-pseudo-orbits for arbitrarily small $\varepsilon>0$. This set is certainly the largest candidate (any other point belongs to the basin of a trapping region, as it was proved by Conley, see the next section). There are several other choices between $\mathcal{P} \operatorname{er}(f)$ and $\mathcal{R}(f)$ but we will only mention the non-wandering set $\Omega(f)$ since it played an important historical role (the reason is that by Pugh's closing lemma $\mathcal{P} \operatorname{er}(f)=\Omega(f)$ for $C^{1}$-generic diffeomorphisms). Recently, the equality $\mathcal{P} \operatorname{er}(f)=\mathcal{R}(f)$ was shown in $\left[\mathrm{BQ}\right.$ for $C^{1}$-generic diffeomorphisms, implying that in some sense the non-trivial dynamics is well defined for the generic diffeomorphisms

Using the genericity of Kupka-Smale diffeomorphisms, Takens showed in [T] that in $\operatorname{Diff}^{r}(M)$ the $\mathcal{P}$ er-tolerance stable diffeomorphisms are generic (he proved a stronger statement, see theorem 8 at section 3.2). From Pugh's closing lemma he got also the genericity of the $\Omega$-tolerance stability in $\operatorname{Diff}^{1}(M)$. The case of the $\mathcal{R}$-tolerance stability is easier and follows from a standard argument?.

Little progress have been done since Takens papers: using the classical shadowing lemma, Robinson [Rob ${ }_{4}$ has proved the conjecture for Axiom A diffeomorphisms. In the space of homeomorphisms $\operatorname{Diff}^{0}(M)$, Mazur has obtained Maz a strong form of the conjecture, improving a partial result of Odani [O].

The tolerance stability conjecture in $\operatorname{Diff}^{1}(M)$ and Takens criterion were a motivation for proving the perturbation results and the generic weak shadowing properties of this paper. Theorem 1 seems very close to show that for $C^{1}$-generic diffeomorphisms $f$, one has $\mathbf{E} \operatorname{Orb}(f)=\operatorname{Orb}(f)$ : it claims that for a $C^{1}$-generic diffeomorphism $f$ and for any $\delta>0$, any extended orbit of $f$ is $\delta$-close to a segment of orbit of $f$ for the Hausdorff distance. There remains however a serious gap which consists in replacing the segments of orbits of $f$ by whole orbits: let us considers the invariant compact subsets of $M$ that are limit of segments of orbits for the Hausdorff distance; this gives a new element $\mathbf{F O r b}(f)$ in $\mathcal{K}(\mathcal{K}(M))$. We always have the inclusions $\operatorname{Orb}(f) \subset \mathbf{F O r b}(f) \subset \mathbf{E O r b}(f)$ but theorem [1 implies that for a $C^{1}$-generic diffeomorphism $f$, one has $\mathbf{F O r b}(f)=\mathbf{E O r b}(f)$. Another consequence is that

5 Due to this result, my opinion is that the chain-recurrent set should now be preferred to the non-wandering set. Note for example that, according to Newhouse $\left[\mathbb{N}_{1}\right]$, the diffeomorphisms that are Axiom A (i.e. the diffeomorphisms such that $\Omega(f)$ is hyperbolic and $\Omega(f)=\mathcal{P} \operatorname{er}(f)$ ) and satisfy the non-cycle condition may be simply defined as the diffeomorphisms whose chain-recurrent set is hyperbolic.

6 One first proves that the map $f \mapsto \mathcal{R}(f)$ is upper-semi-continuous (for any map $g$ close to $f$, the set $\mathcal{R}(g)$ is contained in a neighborhood of $\mathcal{R}(f))$; Baire's theorem implies that the continuity points of this map are residual, see also section 3 . 
the continuity points of the map $g \mapsto \mathbf{F O r b}(g)$ are generic in $\operatorname{Diff}^{1}(M)$. So we improved Takens criterion:

Criterion 2. - If the set of diffeomorphisms $f$ such that $\operatorname{Orb}(f)=$ $\operatorname{FOrb}(f)$ is residual in $\operatorname{Diff}^{1}(M)$, then, the tolerance stability conjecture holds in $\operatorname{Diff}^{1}(M)$.

The reader may observe that by theorem 2, for any $C^{1}$-generic diffeomorphism $f$, and any extended orbit $\mathcal{X}$ of $f$, there are orbits $\left(\mathcal{O}_{n}\right)$ of $C^{1}$-small perturbations $\left(g_{n}\right)$ of $f$ whose closure are arbitrarily close to $\mathcal{X}$ in the Hausdorff topology. This is however not sufficient to obtain $\operatorname{Orb}(f)=$ $\mathbf{F O r b}(f)=\mathbf{E O r b}(f)$ for $C^{1}$-generic diffeomorphisms by some Baire argument since the obtained orbits $\mathcal{O}_{n}$ are not robust under perturbations: for any generic diffeomorphism $g$ close to a perturbation $g_{n}$, all the orbits that shadows $\mathcal{X}$ could escape after a long time and visit other regions of $M$. One would obtain however a control on the asymptotic behavior of the orbits $\mathcal{O}_{n}$ if the orbit $\mathcal{O}(x)$ in theorem 2 can be obtained as a heteroclinic orbit between two hyperbolic periodic orbits. This would be implied by another perturbation result:

Problem 1 (Asymptotic closing lemma). - Let $f$ be a diffeomorphism of a compact manifold $M, \mathcal{U}$ a $C^{1}$-neighborhood of $f$ and $x$ a point in $M$.

- Does there exist a perturbation $g \in \mathcal{U}$ such that $x$ is on the stable manifold of a hyperbolic periodic orbit $\mathcal{O}$ ?

- Can we require moreover that the closure of the forward orbits of $x$ by $f$ and by $g$ remain close for the Hausdorff distance? that the w-limit set of the orbit of $x$ by $f$ (i.e. the accumulation set of the forward orbit of $x)$ and the periodic orbit $\mathcal{O}$ by $g$ are close for the Hausdorff distance?

A positive answer to these questions would also show that for a $C^{1}$-generic diffeomorphism, the stable and unstable manifolds of the hyperbolic periodic points are dense in the manifold.

\subsection{Spectral decomposition of $C^{1}$-generic diffeomorphisms}

In dynamical systems the periodic orbits play a particular role. Some dynamical invariants are associated to them; in general, they also can be followed after perturbation of the dynamics. Moreover, Pugh's closing lemma implies that any non-wandering point of a $C^{1}$-generic diffeomorphism is the limit of a sequence of periodic point. However, since the perturbation is local, 
it does not control the support of the periodic orbit. We will see below that it is interesting to answer the following global problem:

Question. - What is the class of compact sets that may be approximated by a sequence of periodic orbits?

A partial answer was given by Arnaud in [A, She showed that the $\omega$-limit sets of a $C^{1}$-generic diffeomorphism belong to this class of sets. However it is not known if for a generic diffeomorphism the $\omega$-limit sets are the only possible compact sets in this class: in general the set of $\omega$-limit sets is not closed for the Hausdorff topology. Let us also mention Mañé's ergodic closing lemma $\mathrm{M}_{2}$ which gives the measure theoretical viewpoint on the approximation by periodic orbits: it asserts that any ergodic invariant probability measure $\mu$ of a $C^{1}$-generic diffeomorphism is the limit of a sequence of invariant measures supported by periodic orbits $\left(\mathcal{O}_{n}\right)$. Moreover, the orbits $\mathcal{O}_{n}$ converge towards the support of $\mu$ for the Hausdorff topology. For the $C^{1}$ generic diffeomorphisms, theorem 3 provides us with a complete answer of the question, in terms of chain-transitivity.

Theorem 4. - There exists a $G_{\delta}$ dense subset $\mathcal{G}_{\text {rec }}$ of $\operatorname{Diff}^{1}(M)$ such that for any diffeomorphism $f \in \mathcal{G}_{\mathbf{r e c}}$, a compact invariant set $\mathcal{X}$ is the limit (for the Hausdorff distance) of a sequence of periodic orbits if and only if $\mathcal{X}$ chain-transitive.

This result has some consequences on the spectral decomposition of generic diffeomorphisms. Conley has given in [C] a very simple and general way to decompose the chain-recurrence set $\mathcal{R}(f)$ into disjoint and invariant compact sets, called the chain-recurrence classes: two points of $M$ belong to a same chain-recurrence class if for any $\varepsilon>0$, they belong to a same $\varepsilon$-pseudo-orbit which is periodic. He proved that there exists a "Lyapunov function" $h$ associated to this decomposition: $h$ is a continuous map from $M$ to $\mathbf{R}$ such that:

- $h$ decreases along the orbits: $h \circ f(x) \leq h(x)$ for each $x \in M$; moreover, the inequality is strict if and only if $x$ does not belong to the chain-recurrence set.

- $h$ is constant on each chain-recurrence class and takes distinct values on distinct classes.

- The image of the chain-recurrent set by $h$ is totally discontinuous. 
This provides us with some "generalized filtration" of the dynamics: for any distinct chain-recurrence classes $E, E^{\prime}$, there exists a trapping region $U$ that separates] $E$ and $E^{\prime}$.

Several recent results showed that this decomposition for $C^{1}$-generic diffeomorphisms shares some of the properties of Smale's spectral decomposition: for hyperbolic diffeomorphisms, each basic piece $E$ is a homoclinic class. More precisely, it contains a periodic points $P$ and $E$ is the closure of all the transverse intersection points between the invariant manifolds of the orbit of $P$. The dynamics in each homoclinic class is transitive; the periodic points are dense. For $C^{1}$-generic diffeomorphisms, the pieces of Conley's decomposition also hold recurrent dynamics: by the result of $[\mathbb{B C}$, the chain-recurrence classes are the maximal weakly transitive sets. Improving a theorem of Carballo, Morales and Pacifico [CMP], it shows also that the chain-recurrence classes $E$ that contain a periodic point $P$ are the homoclinic classes. Little is known for the other classes (which are called aperiodic classes): the examples of Bonatti and Díaz $\left.\mathbb{B D}_{2}\right]$ assert that they exist for generic diffeomorphisms in (non-empty) open subsets of $\operatorname{Diff}^{1}(M)$ when the dimension of $M$ is larger than or equal to 3 . In order to obtain information on the aperiodic classes, it is useful to approximate them by the periodic orbits in the Hausdorff topology. This is again a consequence of theorem 11.

Corollary 1. - There exists a $G_{\delta}$ dense subset $\mathcal{G}_{\text {aper }}$ of $\operatorname{Diff}^{1}(M)$ such that for any diffeomorphism $f \in \mathcal{G}_{\text {aper }}$, the homoclinic classes of $f$ are dense among the chain-recurrence classes for the Hausdorff topology.

As an application of this theorem, we extend with Abdenur and Bonatti AbBC] a dichotomy proven for homoclinic classes by Bonatti, Díaz and $\mathrm{Pu}-$ jals [BDP to any chain-recurrence class $E$ : either $E$ is the limit of a sequence of periodic sinks or sources for the Hausdorff topology (Newhouse's phenomenon) or $E$ has some weak form of hyperbolicity (a non-trivial dominated splitting of its tangent bundle). Corollary 1 is also used in [ABCD] for discussing the generic dynamics on surfaces.

Theorem 1 also explains how the (non-recurrent) dynamics of a $C^{1}$ generic diffeomorphism is organized between the chain-recurrence classes: if $E_{1}, \ldots, E_{r}$ are chain-recurrence classes, there exists orbits that successively visit arbitrarily small neighborhoods of them if and only if these chainrecurrence classes are connected together by pseudo-orbits with arbitrarily small jumps. in $M \backslash U$.

7 More precisely, $U$ is an open set satisfying $f(\bar{U}) \subset U$; one class is contained in $U$ and the other one 
0.5. Structure of the paper

In the next section, we give a systematic presentation of the various definitions of weak and extended orbits. We also prove some general basic properties of weak orbits.

Section 2 deals with the perturbation techniques in $C^{1}$-dynamics: we recall Hayashi's connecting lemma and the connecting lemma for pseudo-orbits. We then state three perturbation properties that are the technical results of the paper. The first one (approximation by periodic orbits) implies directly theorem 3 (see section 2.4). The two others (approximation by finite segments of orbit and asymptotic approximation) will give theorem 2, as it is shown in section 5.3 .

\section{A.cFenteralizemention of orbits and recurrence}

We discuss in section 3 the generic consequences of these perturbation results: in particular, we prove theorems 1, 4, corollary [1 and criterion 2.

1.1. Ahptatiozss started after motivating discussions with F. Abdenur, M.-C.

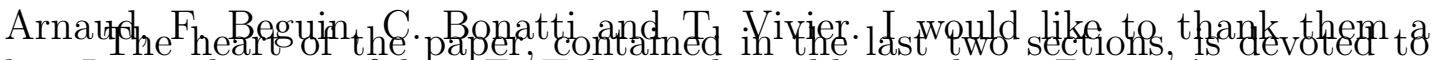

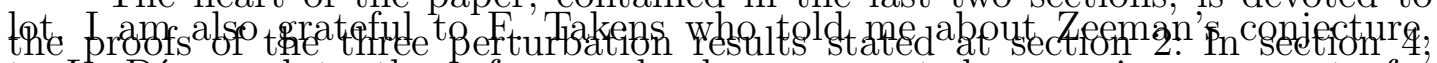

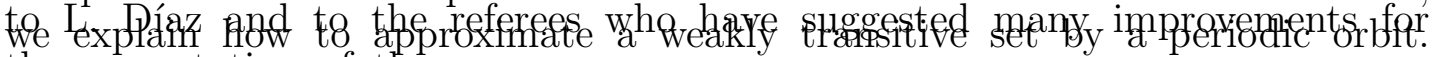
fhe secterenty,

Let $M$ be a compact manifold endowed with a Riemannian metric. The induced distance will be denoted by $\mathrm{d}$. The open ball centered at a point $x \in M$ with radius $\eta>0$ is written $\mathrm{B}(x, \eta)$. The $\eta$-neighborhood of a set $X \subset M$ is the union of the balls $\mathrm{B}(x, \eta)$ over the points $x \in X$. The closure of a subset $X$ in a topological space is $\mathrm{Cl}(X)$. 
The space of non-empty compact sets of $M$ will be denoted by $\mathcal{K}(M)$. This is a compact metric space for the Hausdorff distance defined by:

$$
\mathrm{d}_{H}\left(K, K^{\prime}\right)=\max \left(\max _{x \in K} \mathrm{~d}\left(x, K^{\prime}\right), \max _{x^{\prime} \in K^{\prime}} \mathrm{d}\left(x^{\prime}, K\right)\right) .
$$

If $\mathrm{d}_{H}\left(K, K^{\prime}\right)$ is less than $\eta$, we say that the compact subsets $K$ and $K^{\prime}$ of $M$ are $\eta$-close.

The set of non-empty compact subsets of $\mathcal{K}(M)$ also may be endowed with the Hausdorff topology. This defines a compact metric space $\mathcal{K}(\mathcal{K}(M))$. One adds sometimes an isolated point, the empty set $\emptyset$ of $\mathcal{K}(M)$, and one considers the space $\mathcal{K}(\mathcal{K}(M)) \cup\{\emptyset\}$.

In this work, we consider the space $\operatorname{Diff}^{1}(M)$ of $C^{1}$-diffeomorphisms of $M$ endowed with the $C^{1}$-topology.

\subsection{Generalized iterates}

In this section we will discuss how to generalize the following relation between points of $M: y$ is a forward iterate of $x$ by $f$ if there exists $n \geq 1$ such that $f^{n}(x)=y$. We will give several examples at section 1.5.

1.2.1. We are interested by relations that are closed for the topology of $M$. This leads to the following natural notion of weak iterate, first introduced by Arnaud [A] and Gan and Wen [GW].

Definition 1. - A point $y$ is a weak iterate of $x$ by $f$ (one denotes it by $x \prec y$ ) if for any neighborhoods $U$ of $x$ and $V$ of $y$, there exists $n \geq 1$ and a point $z \in U$ such that $f^{n}(z)$ belongs to $V$.

Sometimes one localizes the dynamics:

- Let $W$ be an open set. If $x$ and $y$ belong to $W$, one defines the relation $x \prec_{W} y$ if for any neighborhoods $U$ and $V$ of $x$ and $y$, there exists $n \geq 1$ and a point $z \in U$ such that $f^{n}(z)$ belongs to $V$ and such that the segment of orbit $\left(z, f(z), \ldots, f^{n}(z)\right)$ is contained in $W$.

- Let $K$ be a compact set. If $x$ and $y$ belong to $K$, one defines the relation $x \prec_{K} y$ if for any neighborhood $W$ of $K$ one has $x \prec_{W} y$.

If $\mathcal{X}$ is a compact set, the relation $x \prec \mathcal{X}$ will mean that for any point $x^{\prime} \in \mathcal{X}$, we have $x \prec x^{\prime}$. One defines in the same way the relations $x \prec_{W} \mathcal{X}$ and $x \prec_{K} \mathcal{X}$.

The relations $\prec, \prec_{W}, \prec_{K}$ are in general not transitive (cf. example 1 of section 1.5). We prove that the relation $\prec_{K}$ is closed: 
Proposition 1. - Let us consider a sequence of compact sets $\left(K_{k}\right)$ and two sequences $\left(x_{k}\right)$ and $\left(y_{k}\right)$ in $M$ such that $x_{k} \prec_{K_{k}} y_{k}$ for each $k$. If $\left(x_{k}\right)$, $\left(y_{k}\right)$ converge towards $x, y$ and if $\left(K_{k}\right)$ converges towards $K$ in the Hausdorff topology, then $x \prec_{K} y$.

Proof. - One considers two neighborhoods $U$ and $V$ of $x$ and $y$ respectively and $W$ a neighborhood of $K$. For $k$ large, $x_{k}$ belongs to $U, y_{k}$ to $V$ and $W$ is a neighborhood of $K_{k}$. Since $x_{k} \prec_{K_{k}} y_{k}$, there exists a segment of orbit that crosses $U$ and then $V$ and is contained in $W$ what was to be shown.

The relation $\prec_{K}$ is invariant by $f$, by $f^{-1}$ and moreover $x \prec_{K} y$ implies $x \prec_{K} f(y)$. We also have the following property:

Proposition 2. - Let $f$ be a diffeomorphism and $K$ a compact set. Let $x, y$ be two points in $K$ such that $x \prec_{K} y$. If $f(x) \neq y$ then $f(x) \prec_{K} y$ holds also.

Proof. - Let $W$ be a neighborhood of $K$ and $U, V$ be two neighborhoods of $f(x)$ and $y$ respectively. Since $f(x)$ is different from $x$, one may assume that $U$ and $V$ are disjoint. Using the relation $x \prec_{K} y$, there exists a segment of orbit $\left(z_{0}, \ldots, z_{n}\right)$ in $W$ with $n \geq 1$ such that $z_{0}$ belongs to $f^{-1}(U)$ and $z_{n}$ to $V$. Since $U$ is disjoint from $V, z_{1}$ does not belong to $V$ and $n$ is larger than or equal to 2 . Hence $\left(z_{1}, \ldots, z_{n}\right)$ is a finite segment of orbit (not reduced to a point) contained in $W$ such that $z_{1}$ belongs to $U$ and $z_{n}$ to $V$. This shows $f(x) \prec_{W} y$. Since $W$ is any neighborhood of $K$, we have $f(x) \in K$ and $f(x) \prec_{K} y$.

1.2.2. When we consider perturbations of the dynamics, one may want to work with a relation which is semi-continuous with respect to the map $f$. Introducing the pseudo-orbits, one obtains the following definition.

Definition 2. - A point $y$ is a chain-iterate of $x$ by $f$ (one denotes it by $x \dashv y)$ if for any $\varepsilon>0$, there exists an $\varepsilon$-pseudo-orbit $\left(z_{0}, \ldots, z_{n}\right)$ (with $n \geq 1$ ) such that $z_{0}=x$ and $z_{n}=y$.

8 Since we are interested here by the semi-continuity property of the relation with respect to $f$, one could define the following relation: $y$ is a "generalized iterate" of $x$ by $f$ if there exists a sequence $\left(f_{k}\right)$ which converges toward $f$ for an appropriate topology, two sequences of points $\left(x_{k}\right)$ and $\left(y_{k}\right)$ which converge in $M$ toward $x$ and $y$ respectively, and a sequence of integers $\left(n_{k}\right)$ such that for each $k$, we have $n_{k} \geq 1$ and $f^{n}\left(x_{k}\right)=y_{k}$. The definition of "chain-iterates" which uses pseudo-orbits appears naturally when one considers rather random perturbations of the dynamics. It is easier to work with the definition of chain-iterate since it has the advantage to involve only the initial dynamics $f$ (and not to depend on the choice of a topology on the set of the dynamical systems). 
Let $E$ be any subset of $M$. If $x$ and $y$ belong to $E$, one defines the relation $x \dashv_{E} y$ if for any $\varepsilon>0$, there exists an $\varepsilon$-pseudo-orbit $\left(z_{0}, \ldots, z_{n}\right)$ (with $n \geq 1$ ) contained in $E$ such that $z_{0}=x$ and $z_{n}=y$.

These relations are clearly transitive. Moreover the relation $x \prec_{K} y$ implies the relation $x \dashv_{K} y$ (the converse is false, see example 1). The following proposition shows in particular that if the set $E$ is closed, then the relation $\dashv_{E}$ is closed.

Proposition 3. - Let us consider a sequence $\left(f_{k}\right)$ of diffeomorphisms that converges towards $f \in \operatorname{Diff}^{1}(M)$. One also considers a sequence of compact sets $\left(K_{k}\right)$ and two sequences $\left(x_{k}\right)$ and $\left(y_{k}\right)$ in $M$ such that for each $k$, the point $y_{k}$ is a chain-iterate of $x_{k}$ by $f_{k}$. If $\left(x_{k}\right)$ converges towards $x$ and $\left(y_{k}\right)$ towards $y$ and if $\left(K_{k}\right)$ converges towards $K$ in the Hausdorff topology, then $y$ is a chain-iterate of $y$ by $f$.

Proof. - One fixes $\varepsilon>0$. For $k$ large enough, $\mathrm{d}_{H}\left(K_{k}, K\right), \mathrm{d}\left(x_{k}, x\right)$, $\mathrm{d}\left(y_{k}, y\right)$ and the $C^{0}$-distance between $f_{k}$ and $f$ are small. We consider a $\eta$ pseudo-orbit of $f_{k}$ in $K_{k}$ that connects $x_{k}$ to $y_{k}$. For $\eta$ small enough, it is shadowed by an $\varepsilon$-pseudo-orbit in $K$ that connects $x$ to $y$ what was to be shown.

\subsection{Different kinds of recurrence}

We now discuss the various definitions for a compact invariant set $K$ to support recurrent dynamics.

1.3.1. The sets that satisfy the strongest form of recurrence are the periodic orbits. Since one looks for a notion which is closed for the Hausdorff topology, one considers all the compact sets that are limit of periodic orbits in the Hausdorff topology. This collection defines an element of $\mathcal{K}(\mathcal{K}(M)) \cup$ $\{\emptyset\}$ which is denoted by $\operatorname{Per}(f)$.

1.3.2. In the introduction, we defined the notion of weakly transitive set巴एم:

9 We do not distinguish here between the cases where $E$ is open or closed. The reason is that if one considers an $\varepsilon$-pseudo-orbit that joints two points $x, y$ of $E$ and that can be chosen arbitrarily close to $E$, then, for any $\varepsilon^{\prime}>\varepsilon$, there also exists an $\varepsilon^{\prime}$-pseudo-orbit that joints $x$ to $y$ and that is contained in $E^{\prime}$. It is obtained by "projecting" the points of the first orbit to the set $E$.

10 Note that a slightly different notion of weak transitivity was proposed in $\mathrm{A}_{1}$ : it requires only that for any open sets $U, V$ which intersect $\mathcal{X}$, there exists $n \geq 1$ and a point $z \in U$ such that $f^{n}(z)$ belongs to $V$. In particular, the union of two different periodic orbits, contained in a same transitive set, satisfies this last condition but not our definition of weak transitivity. 
Definition 3. - A closed set $\mathcal{X} \subset M$ which is invariant by $f$ is weakly transitive if for any neighborhood $W$ of $\mathcal{X}$, and any open sets $U, V$ which intersect $\mathcal{X}$, there exists $n \geq 1$ and a point $z \in U$ such that $f^{n}(z)$ belongs to $V$ and such that the segment of orbit $\left(z, f(z), \ldots, f^{n}(z)\right)$ is contained in $W$.

This can be compared to the classical definition of transitivity: $\mathcal{X}$ is transitive if for any open sets $U$ and $V$ which intersect $\mathcal{X}$, there exists $n \geq 1$ and a point $z \in U \cap \mathcal{X}$ such that $f^{n}(z)$ belongs to $V$. By proposition $\mathbb{1}$, the set of weakly transitive sets is closed in $\mathcal{K}(M)$. Hence, this is an element of $\mathcal{K}(\mathcal{K}(M))$ which is denoted by $\mathbf{W T r a n s}(f)$.

One can give an equivalent definition using the relation $\prec \mathcal{X}$ : the set $\mathcal{X}$ is weakly transitive if and only if for any points $x, y$ in $\mathcal{X}$, we have $x \prec_{\mathcal{X}} y$.

1.3.3. Replacing $\prec_{\mathcal{X}}$ by the relation $\dashv_{\mathcal{X}}$ in the definition of weakly transitive set, one gets another definition:

Definition 4. - A closed set $\mathcal{X} \subset M$ which is invariant by $f$ is chaintransitive if for any points $x, y$ in $\mathcal{X}$ one has $x \dashv_{\mathcal{X}} y$.

By proposition 3, the sets of chain-transitive sets is closed in $\mathcal{K}(M)$, defining an element of $\mathcal{K}(\mathcal{K}(M))$ which is denoted by CTrans $(f)$. Moreover, it is upper-semi-continuous with respect to $f$ : if $\left(f_{n}\right)$ converges towards $f$ in the $C^{0}$-topology, then, the upper-limit of the compact sets $\operatorname{CTrans}\left(f_{n}\right)$ is contained in CTrans $(f)$.

Note that the chain-recurrence classes defined at section 0.4 are the chain-transitive sets that are maximal for the inclusion. Two chain-recurrence classes are disjoint or equal since they also are the equivalence classes for the following relation in $\mathcal{R}(f)$ : for any points $x, y \in \mathcal{R}(f)$, we define $x \mapsto y$ if $x \dashv y$ and $y \dashv x$.

From the construction, we always have the inclusions

$$
\operatorname{Per}(f) \subset \mathbf{W T r a n s}(f) \subset \mathbf{C T r a n s}(f) .
$$

In general they do not coincide (cf. the examples 2 and 3 ).

In dynamics, one also often considers the collections of transitive sets, and of limit sets (the accumulation points of orbits). The collection of periodic orbits is contained in the collection of transitive sets which is contained in the collection of limit sets. All of them are contained in WTrans $(f)$. In general neither the collection of limit sets, nor the collection of transitive sets are closed; moreover, there is no relation with $\operatorname{Per}(f)$ for the inclusion (cf. examples 0 and 2). 


\subsection{The generalized orbits}

Similarly to the various definitions of generalized iterates, one now introduces different generalizations of the orbits.

1.4.1. In the strongest form, one simply considers the collection of invariant compact sets that are limit of closure of orbits by $f$ and one obtains an element $\operatorname{Orb}(f)$ of $\mathcal{K}(\mathcal{K}(M))$.

1.4.2. The invariant compact sets that are limit of finite segments of orbits in general produce a different element $\mathbf{F O r b}(f) \in \mathcal{K}(\mathcal{K}(M)$ ) (see example 3).

1.4.3. More generally, from any definition of generalized iterates, one derives a definition of generalized orbit: a set $K$ is a generalized orbit if for any pair of point $x, y$ in $K$, one is a generalized iterate of the other. In particular one gets the following definitions:

Definition 5. - An invariant compact set $\mathcal{X}$ is a weak orbit (resp. an extended orbit) if for any points $x \neq y$ in $\mathcal{X}$ one has $x \prec \mathcal{X} y$ or $y \prec \mathcal{X} x$ (resp. one has $x \dashv_{\mathcal{X}} y$ or $\left.y \dashv_{\mathcal{X}} x\right)$.

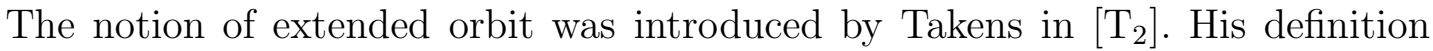
is equivalent to ours: $E$ is an extended orbit if and only if for any $\varepsilon>0$, the compact set $E$ is approximated in the Hausdorff topology by the closures of $\varepsilon$-pseudo-orbits.

By propositions 1 and 3, the sets of weak orbits and of extended orbits are closed for the Hausdorff topology, defining two elements of $\mathcal{K}(\mathcal{K}(M))$ that we denote by $\mathbf{W O r b}(f)$ and $\mathbf{E O r b}(f)$ respectively. We always have the inclusions

$$
\operatorname{Orb}(f) \subset \mathbf{F O r b}(f) \subset \mathbf{W O r b}(f) \subset \mathbf{E O r b}(f)
$$

but in general these sets do not coincide.

It is also useful to introduce the generalized segments of orbits: in the definition of the elements of $\mathbf{F O r b}(f), \mathbf{W O r b}(f)$ and of $\mathbf{E O r b}(f)$, one may also consider compact sets that are not necessarily invariant by $f$. This defines the following elements of $\mathcal{K}(\mathcal{K}(M))$ :

$$
\operatorname{Seg}(f) \subset \mathbf{W S e g}(f) \subset \mathbf{E S e g}(f) .
$$

The elements of $\mathbf{W S e g}(f)$ will be called the weak segments of orbits; those of $\operatorname{ESeg}(f)$, the extended segments of orbits.

By proposition 3, the sets $\mathbf{E O r b}(f)$ and $\mathbf{E S e g}(f)$ vary upper-semicontinuously with respect to the dynamics $f$. For $\mathbf{F O r b}(f)$ and $\mathbf{S e g}(f)$, we have: 
Proposition 4. - The maps $f \mapsto \mathbf{S e g}(f)$ and $f \mapsto \mathbf{F O r b}(f)$ are lowersemi-continuous.

Proof. - Let $\mathcal{X}$ be any element of $\mathbf{S e g}(f)$. It is arbitrarily close to a segment of orbit $\left\{x, f(x), \ldots, f^{n}(x)\right\}$ for the Hausdorff topology. For $g$ close to $f$, the orbit $\left\{x, g(x), \ldots, g^{n}(x)\right\}$ belongs to $\operatorname{Seg}(g)$ and is close to $\left\{x, f(x), \ldots, f^{n}(x)\right\}$, hence, close to $\mathcal{X}$, showing the lower-semi-continuity of $f \mapsto \operatorname{Seg}(f)$. One obtains the result for $\mathbf{F O r b}(f)$ just by noting that $\mathbf{F O r b}(f)$ coincides with the subset of $\operatorname{Seg}(f)$ whose elements are invariant by $f$.

Note also that we have the inclusions

$$
\begin{gathered}
\operatorname{Per}(f) \subset \operatorname{Orb}(f) \subset \mathbf{F O r b}(f) \subset \mathbf{S e g}(f), \\
\operatorname{WTrans}(f) \subset \mathbf{W O r b}(f) \subset \mathbf{W S e g}(f), \\
\mathbf{C T r a n s}(f) \subset \operatorname{EOrb} \subset \mathbf{E S e g}(f) .
\end{gathered}
$$

1.5. Examples

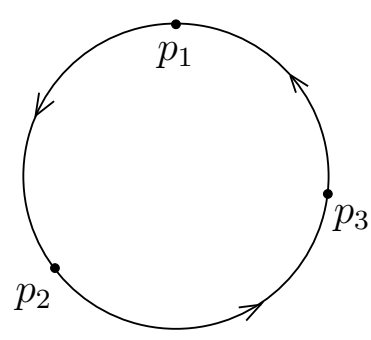

$f_{1}$

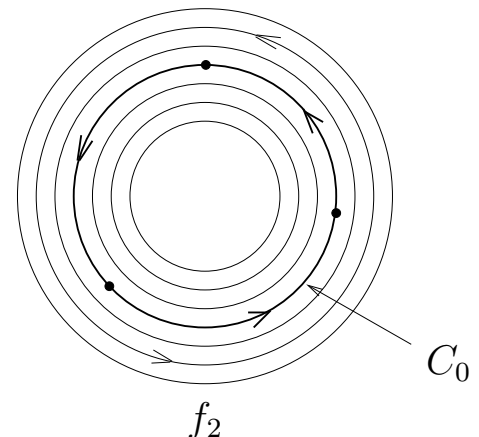

FIG. 1. -

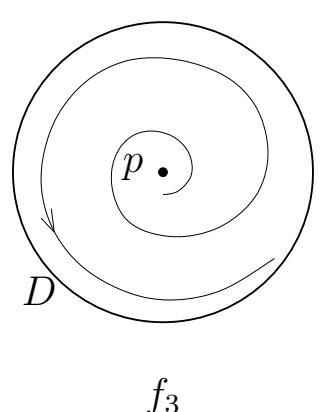

$f_{3}$

0. Among all the elements of $\mathcal{K}(\mathcal{K}(M)) \cup\{\emptyset\}$ defined above, $\operatorname{Per}(f)$ is the only one that can be the empty set: this happens for instance with the irrational rotations of the circle. In this case, the collection of transitive sets (here the whole circle) is not contained in $\operatorname{Per}(f)$.

1. Let $f_{1}$ be an orientation-preserving diffeomorphism of the circle $\mathbf{T}^{1}$ having three semi-stable fixed points $p_{1}, p_{2}$ and $p_{3}$ (see the first example of figure (1)). The set $\operatorname{Per}\left(f_{1}\right)$ equals $\left\{\left\{p_{1}\right\},\left\{p_{2}\right\},\left\{p_{3}\right\}\right\}$. Up to reordering the three fixed points, one has $p_{1} \prec p_{2}, p_{2} \prec p_{3}$ and $p_{3} \prec p_{1}$ but the other 
relations between these points are not satisfied: in particular $p_{1} \prec p_{3}$ is false showing that in this case $\prec$ is not transitive and that $\prec$ and $\dashv$ may differ.

2. Let $f_{2}$ be a diffeomorphism of the torus, foliated by circles $\left(C_{t}\right)_{t \in \mathbf{T}^{1}}$, such that (see the second example of figure 11)):

- On one circle, $C_{0}$, the induced dynamics coincides with the former example.

- On the circles $C_{t} \neq C_{0}$ the dynamics is conjugate to a rotation.

- The rotation number $\rho_{t}$ on the circles $C_{t}$ is monotonic in $t$.

In this example, the elements of $\operatorname{Per}\left(f_{2}\right)=\mathbf{W T r a n s}\left(f_{2}\right)$ are the periodic orbits of $f_{2}$ and each circle $C_{t}$. In particular $C_{0}$ is in $\operatorname{Per}\left(f_{2}\right)$, but is not a limit set; the set $C_{0}$ is weakly transitive but is not transitive. Note also that despite $C_{0}$ is weakly transitive for $f_{2}$, the dynamics $f_{1}$ induced by $f_{2}$ on $C_{0}$ is not weakly transitive.

The set CTrans $\left(f_{2}\right)$ is larger: it contains for example the whole torus.

3. Let $f_{3}$ be a surface diffeomorphism which coincides with the identity outside an open disk $D$. In $D, f_{3}$ has a fixed point $p$. Any other orbit in $D$ converges towards $p$ in the past and accumulates on the whole boundary of $D$ in the future (see the third example of figure 11)). On this example, the boundary of $D$ belongs to $\mathbf{F O r b}\left(f_{3}\right)$ but not to $\operatorname{Orb}\left(f_{3}\right)$. The boundary of $D$ belongs to $\mathbf{W T r a n s}\left(f_{3}\right)$ but not to $\operatorname{Per}\left(f_{3}\right)$.

\subsection{Properties of weak orbits}

1.6.1. Weak orbits and periodic points. - The periodic orbits are the simplest weak orbits. We analyze here the other weak orbits. (Recall that a diffeomorphism satisfies condition (A) if for any integer $n \geq 1$, the fixed points of $f^{n}$ are isolated.)

Lemma 1. - Let $\mathcal{X} \in \mathbf{W S e g}(f)$ be a weak segment of orbit of a diffeomorphism $f$ that satisfies condition $(A)$. If $\mathcal{X}$ is not a periodic orbit, the non-periodic points are dense in $\mathcal{X}$.

Proof. - We first claim that the periodic orbits are not isolated in $\mathcal{X}$. The proof is by contradiction: let us consider a periodic isolated point $x$ in $\mathcal{X}$; the periodic orbit $\mathcal{O}$ of $x$ is isolated from $\mathcal{X} \backslash \mathcal{O}$. By our assumption on $\mathcal{X}$, the set $\mathcal{X} \backslash \mathcal{O}$ is non-empty and we will see that this gives a contradiction. One can choose two neighborhoods $U$ and $V$ of $\mathcal{O}$ and $\mathcal{X} \backslash \mathcal{O}$ respectively such that $f(U) \cap V$ and $f(V) \cap U$ are both empty. By definition of weak segment of orbit, there exists a finite segment of orbit contained in the neighborhood 
$U \cup V$ of $\mathcal{X}$ that crosses $U$ and $V$. This is impossible by our choice of $U$ and $V$. This shows the claim.

Let us now consider the set $P_{n}$ of periodic points contained in $\mathcal{X}$ with period $n$. This set is closed and finite by the assumption (A) on the diffeomorphism. Since the points of $P_{n}$ are not isolated in $\mathcal{X}$, the set $P_{n}$ has empty interior in $\mathcal{X}$. By Baire's theorem the complement of the union $\cup_{n} P_{n}$ is dense in $\mathcal{X}$. Hence, the non-periodic points are dense.

Proposition 5. - Let $\mathcal{X}$ be a weak orbit of a diffeomorphism $f$ that satisfies condition (A). If $\mathcal{X}$ is not a periodic orbit, then for any $\eta_{0}>0$ there exists a subset $X$ of $\mathcal{X}$ which has the following properties:

1. $X$ is finite (and contains at least two points);

2. the Hausdorff distance between $\mathrm{Cl}\left(\cup_{n} f^{n}(X)\right)$ and $\mathcal{X}$ is less than $\eta_{0}$;

3. $X$ has no periodic point;

4. the orbits of any two distinct points of $X$ are disjoint.

Proof. - By compactness of $\mathcal{X}$ and by lemma 1, there exists a finite subset $X$ of non-periodic points in $\mathcal{X}$ which is $\eta_{0}$-close to $\mathcal{X}$. Up to removing some points of $X$, the orbit of $X$ is $\eta_{0}$-close to $\mathcal{X}$ but $X$ intersects each orbit of $\mathcal{X}$ in at most one point. This gives the result.

1.6.2. Weak orbits and maximal invariant subsets. - Any weak orbit $\mathcal{X}$, such that $\prec \mathcal{X}$ is an ordering, contains points $y$ that are maximal for $\prec_{\mathcal{X}}$ :

Proposition 6. - Let be $\mathcal{X}$ a weak orbit such that the relation $\prec \mathcal{X}$ is transitive. Then, $\mathcal{X}$ contains an invariant closed set $A$ such that for any $x \in \mathcal{X}$ and $y \in A$, one has $x \prec \mathcal{X} y$.

Remark. - Any non-empty subset of $A$ will also satisfy the proposition. Hence, one may choose $A$ minimal for the inclusion, so that the induced dynamics on $A$ is minimal.

Proof. - One considers the family $\mathcal{F}$ of closed and invariant non-empty subsets $K$ of $\mathcal{X}$ such that for any $x \in \mathcal{X} \backslash K$ and $y \in K$ one has $x \prec \mathcal{X} y$. This family is non-empty (it contains $\mathcal{X}$ ) and partially ordered by inclusion. One easily checks that Zorn's lemma can be applied and one considers a minimal element $A \subset \mathcal{X}$. We will now prove that for any points $x, y$ in $A$, one has $x \prec \mathcal{X} y$. This will concludes the proof.

Let us consider two points $x, y$ in $A$ and assume by contradiction that $x \prec \mathcal{X} y$ does not hold. One then considers the set $A^{\prime}$ of points $z \in A$ such 
that $x \prec \mathcal{X} z$. This set is closed (proposition 11), non-empty (it contains $\omega(x)$ ) and moreover $f\left(A^{\prime}\right) \subset A^{\prime}$. It is smaller than $A$ since $y$ belongs to $A \backslash A^{\prime}$. Since $\mathcal{X}$ is a weak orbit, for any point $z^{\prime} \in A^{\prime}$ and any point $z \in A \backslash A^{\prime}$ one has $z \prec \mathcal{X} z^{\prime}$ or $z^{\prime} \prec_{\mathcal{X}} z$. However, the second case is impossible: by transitivity of $\prec_{\mathcal{X}}$, one would get $x \prec \mathcal{X} z$ so that $z \in A^{\prime}$ by definition of $A^{\prime}$. This contradicts our choice of $z$. Hence $z \prec \mathcal{X} z^{\prime}$. For any point $z^{\prime} \in A^{\prime}$ and any point $z \in \mathcal{X} \backslash A$ one also has $z \prec \mathcal{X} z^{\prime}$ by definition of $A$. We proved that for any $z \in \mathcal{X} \backslash A^{\prime}$ and any $z^{\prime} \in A^{\prime}$, one has $z \prec \mathcal{X} z^{\prime}$.

Let us define $A_{0}=\cap_{n \in \mathbf{N}} f^{n}\left(A^{\prime}\right)$. Since $f\left(A^{\prime}\right) \subset A^{\prime}$, the set $A_{0}$ is the intersection of a decreasing sequence of non-empty compact sets. Hence, $A_{0}$ is non-empty, compact and invariant. Moreover, for any $z \in \mathcal{X} \backslash A_{0}$ and $z^{\prime} \in A_{0}$, there exists $n \in \mathbf{N}$ such that $f^{-n}(z)$ belongs to $\mathcal{X} \backslash A^{\prime}$ and $f^{-n}\left(z^{\prime}\right)$ to $A_{0} \subset A^{\prime}$. One thus gets $f^{-n}(z) \prec \mathcal{X} f^{-n}\left(z^{\prime}\right)$ so that $z \prec \mathcal{X} z^{\prime}$. Hence $A_{0}$ is in the family $\mathcal{F}$ of compact sets defined above and $A$ is not a minimal element of $f$. This is a contradiction. This ends the proof.

1.6.3. Weak orbits that are not weakly transitive. - We now analyze weak orbits that are not weakly transitive.

Proposition 7. - Let $\mathcal{X}$ a weak orbit that is not weakly transitive and such that the relation $\prec \mathcal{X}$ is transitive. Then, for any $\eta_{0}>0$, there exist two weak orbits $\mathcal{X}_{\alpha}$ and $\mathcal{X}_{\omega}$ whose relations $\prec_{\mathcal{X}_{\alpha}}$ and $\prec_{\mathcal{X}_{\omega}}$ are transitive, and a point $z_{0} \in \mathcal{X}$ such that:

1. $\mathcal{X}_{\alpha}, \mathcal{X}_{\omega}$ and the orbit $\mathcal{O}\left(z_{0}\right)$ of $z_{0}$ are pairwise disjoint and contained in $\mathcal{X}$.

2. The set $\mathrm{Cl}\left(\mathcal{X}_{\alpha} \cup \mathcal{O}\left(z_{0}\right) \cup \mathcal{X}_{\omega}\right)$ is $\eta_{0}$-close to $\mathcal{X}$ in the Hausdorff topology.

3. For any $x \in \mathcal{X}_{\alpha}$ and $z \in \mathcal{X} \backslash \mathcal{X}_{\alpha}$ we have $x \prec \mathcal{X} z$ but the relation $z \prec \mathcal{X} x$ is not satisfied.

4. For any $y \in \mathcal{X}_{\omega}$ and $z \in \mathcal{X} \backslash \mathcal{X}_{\omega}$ we have $z \prec_{\mathcal{X}}$ y but the relation $y \prec \mathcal{X} z$ is not satisfied.

Remark. - By lemma [1, if $f$ satisfies condition (A), the point $z_{0}$ may be modified and chosen non-periodic.

Proof. - One first builds $\mathcal{X}_{\omega}$ : by proposition 6, there exists an invariant closed subset $A$ of $\mathcal{X}$ such that for any $x \in \mathcal{X}$ and $y \in A$ we have $x \prec \mathcal{X} y$. One defines $\mathcal{X}_{\omega}$ as the set of points $y \in \mathcal{X}$ such that for any $x \in A$, one has $x \prec_{\mathcal{X}} y$ and $y \prec_{\mathcal{X}} x$. This set is closed, invariant and contains $A$.

By transitivity of $\prec_{\mathcal{X}}$, for any $x \in \mathcal{X}$ and $y \in \mathcal{X}_{\omega}$ we have $x \prec_{\mathcal{X}}$ $y$. Moreover $y \prec \mathcal{X} x$ implies $x \in \mathcal{X}_{\omega}$. We then prove that $\mathcal{X}_{\omega}$ is a weakly transitive set, hence a weak orbit (whose relation $\prec_{\mathcal{X}_{\omega}}$ is transitive). Since $\mathcal{X}$ is not weakly transitive, this also shows that $\mathcal{X}_{\omega}$ is not equal to $\mathcal{X}$. 
Claim 1. - The set $\mathcal{X}_{\omega}$ is a weakly transitive set.

Proof. - For any two points $x, y \in \mathcal{X}_{\omega}$, we have $x \prec \mathcal{X} y$. Let us assume by contradiction that the relation $x \prec \mathcal{X}_{\omega} y$ is not satisfied: in particular, there are segments of orbits that connect small neighborhoods $U$ of $x$ to small neighborhoods $V$ of $y$, that are contained in arbitrarily small neighborhoods $W$ of $\mathcal{X}$, but that are not contained in arbitrarily small neighborhoods of $\mathcal{X}_{\omega}$. When the size of the neighborhoods $U, V, W$ decreases, these segments of orbits have an accumulation point $z$ which belongs to $\mathcal{X} \backslash \mathcal{X}_{\omega}$. Moreover, we obtain $x \prec_{\mathcal{X}} z \prec_{\mathcal{X}} y$. This implies by transitivity of $\prec_{\mathcal{X}}$ that for any $\tilde{z}$ in $\mathcal{X}_{\omega}$ one has $\tilde{z} \prec \mathcal{X} z$ and $z \prec \mathcal{X} \tilde{z}$. Hence, by definition of $\mathcal{X}_{\omega}$ (and using $A \subset \mathcal{X}_{\omega}$ ), one gets $z \in \mathcal{X}_{\omega}$ which is a contradiction.

One then builds $\mathcal{X}_{\alpha}$ : since $\mathcal{X}_{\omega}$ is not equal to $\mathcal{X}$, one chooses a finite set $\left\{z_{1}, \ldots, z_{r}\right\}$ in $\mathcal{X} \backslash \mathcal{X}_{\omega}$ such that $\left\{z_{1}, \ldots, z_{r}\right\} \cup \mathcal{X}_{\omega}$ is $\eta_{0}$-close to $\mathcal{X}$ in the Hausdorff topology. Using that $\mathcal{X}$ is a weak orbit, one can order this set as

$$
z_{1} \prec_{\mathcal{X}} z_{2} \prec_{\mathcal{X}} \cdots \prec_{\mathcal{X}} z_{r} \prec_{\mathcal{X}} \mathcal{X}_{\omega} .
$$

One denotes by $B$ the set of points $x \in \mathcal{X}$ such that $x \prec \mathcal{X} z_{r}$. This is a closed and non-empty set which satisfies $B \subset f(B)$. The set $\mathcal{X}_{\alpha}$ is defined as the decreasing intersection $\mathcal{X}_{\alpha}=\cap_{n \geq 0} f^{-n}(B)$. This is an invariant and non-empty compact set.

Claim 2. - For any points $x \in \mathcal{X}_{\alpha}$ and $z \in \mathcal{X}$, the relation $z \prec \mathcal{X} x$ implies that $z$ belongs to $\mathcal{X}_{\alpha}$.

Proof. - For any $n \geq 0$, we have $f^{n}(x) \in \mathcal{X}_{\alpha} \subset B$; hence, $f^{n}(x) \prec \mathcal{X} z_{r}$. Using $f^{n}(z) \prec \mathcal{X} f^{n}(x)$, one also gets $f^{n}(z) \prec \mathcal{X} z_{r}$ so that $f^{n}(z)$ belongs to $B$. One has $z \in \cap_{n \geq 0} f^{-n}(B)$ and thus $z \in \mathcal{X}_{\alpha}$, proving the claim.

This claim and the fact that $\mathcal{X}$ is a weak orbit show that for any point $x \in \mathcal{X}_{\alpha}$ and $z \in \mathcal{X} \backslash \mathcal{X}_{\alpha}$, we have $x \prec \mathcal{X} z$. One also gets that $\mathcal{X}_{\alpha}$ is a weak orbit whose relation $\prec \mathcal{X}_{\alpha}$ is transitive: since $\mathcal{X}$ is a weak orbit whose relation is transitive, it is enough to show that for any points $x, y \in \mathcal{X}_{\alpha}$ the relation $x \prec \mathcal{X} y$ implies $x \prec_{\mathcal{X}_{\alpha}} y$. The argument is similar to the proof of the first claim (One argues by contradiction: if $x \prec \mathcal{X}_{\alpha} y$ does not hold, there exists a point $z \in \mathcal{X}$ such that $x \prec_{\mathcal{X}} z \prec_{\mathcal{X}} y$ and such that $z \notin \mathcal{X}_{\alpha}$. This is impossible since $z \prec \mathcal{X} y$ and $y \in \mathcal{X}_{\alpha}$ imply by the second claim that $z$ belongs to $\mathcal{X}_{\alpha}$.)

Let us note that $\mathcal{X}_{\alpha}$ and $\mathcal{X}_{\omega}$ are disjoint. Otherwise, there would exist $y \in B$ such that for any point $x \in \mathcal{X}_{\omega}$ we have $x \prec \mathcal{X} y$. As $y \prec \mathcal{X} z_{r}$ by definition of $B$ we get also $x \prec_{\mathcal{X}} z_{r}$ by transitivity of $\prec_{\mathcal{X}}$. We also have $z_{r} \prec_{\mathcal{X}}$ $x$. These two relations imply that $z_{r}$ belongs to $\mathcal{X}_{\omega}$. This is a contradiction. 
Since $\mathcal{X}$ is a weak orbit and since the two invariant subsets $\mathcal{X}_{\alpha}$ and $\mathcal{X}_{\omega}$ are disjoint, the set $\mathcal{X} \backslash\left(\mathcal{X}_{\alpha} \cup \mathcal{X}_{\omega}\right)$ is non-empty. By the properties proven for $\mathcal{X}_{\alpha}$ and $\mathcal{X}_{\omega}$, we have shown the items 1), 3) and 4) of the proposition. In particular, for any $x \in \mathcal{X}_{\alpha}, y \in \mathcal{X}_{\omega}$ and $z \in \mathcal{X} \backslash\left(\mathcal{X}_{\alpha} \cup \mathcal{X}_{\omega}\right)$,

$$
x \prec \mathcal{X} z \prec \mathcal{X} y \text {. }
$$

One then introduces the point $z_{0}$. Two cases are possible:

- either $z_{r}$ belongs to $\mathcal{X}_{\alpha}$ and the point $z_{0}$ is arbitrarily chosen in $\mathcal{X} \backslash\left(\mathcal{X}_{\alpha} \cup \mathcal{X}_{\omega}\right)$

- or $z_{r}$ does not belong to $\mathcal{X}_{\alpha}$ and one sets $z_{0}=z_{r}$.

It follows directly from this definition that the orbit $\mathcal{O}\left(z_{0}\right)$ of $z_{0}$ is disjoint from $\mathcal{X}_{\alpha} \cup \mathcal{X}_{\omega}$. We now prove that $\mathcal{X}_{\alpha} \cup \mathcal{O}\left(z_{0}\right)$ contains the set $X$ and, by our choice of the set $X$, this will imply the second item of the proposition: let us consider any point $z_{i} \in X$; we have $z_{i} \prec \mathcal{X} z_{r}$. If $z_{r} \in \mathcal{X}_{\alpha}$, the last claim shows that $z_{i}$ is also included in $\mathcal{X}_{\alpha}$. If $z_{i}$ does not belong to the orbit of $z_{r}$, one can apply proposition 2: for each $n \geq 0$, we have $f^{n}\left(z_{i}\right) \prec \mathcal{X} z_{r}$ so that $z_{i}$ again belongs to $\mathcal{X}_{\alpha}=\cap_{n \geq 0} f^{n}(B)$. In remaining case $z_{r} \notin \mathcal{X}_{\alpha}$ but $z_{i}$ belongs to the orbit of $z_{r}=z_{0}$ : we have $z_{i} \in \mathcal{O}\left(z_{0}\right)$. In any of these three cases, one has $x_{i} \in \mathcal{X}_{\alpha} \cup \mathcal{O}\left(z_{0}\right)$ as required.

The proof of proposition 7 is now complete.

\section{Connecting lemmas}

In this section, we recall Hayashi's connecting lemma and the connecting lemma for pseudo-orbits. We then state the technical versions of the perturbation results given in the introduction (theorems 2 and 3).

\subsection{Support of perturbations}

Let $f$ be a diffeomorphism and $\mathcal{U}$ a neighborhood of $f$ in $\operatorname{Diff}^{1}(M)$. Two perturbations $g_{1}, g_{2} \in \mathcal{U}$ of $f$ have disjoint supports if $g_{i}=f$ outside some open set $U_{i}$ for $i=1,2$ and $U_{1} \cap U_{2}=\emptyset$. In this case, one defines the composed perturbation $g$ by $g=f$ outside $U_{1} \cup U_{2}, g=g_{1}$ on $U_{1}$ and $g=g_{2}$ on $U_{2}$.

In general $g$ does not belong to $\mathcal{U}$. However, there exists (see the flexibility of the lift axiom in section 2 of [PR]) a basis of neighborhoods $\mathcal{U}$ of $f$ which have this property:

(F) For any perturbations $g_{1}, g_{2} \in \mathcal{U}$ of $f$ with disjoint supports the composed perturbation $g$ remains in $\mathcal{U}$. 


\subsection{Hayashi's connecting lemma}

In the proof of the perturbation lemmas we will use Hayashi's connecting lemma. The original proof appeared in [H] but other references are given in [WX, $\left.\mathrm{A}_{1}\right]$. We need a slightly more general statement than the version of [A]], in order to perturb at the same time in different domains. However the proof is the same.

Theorem 5 (Hayashi's connecting lemma). - Let $f_{0}$ be a diffeomorphism of a compact manifold $M$, and $\mathcal{U}$ a neighborhood of $f_{0}$ in $\operatorname{Diff}^{1}(M)$. Then there exists $N \geq 1$ such that for any $z \in M$ which is not a periodic orbit of period less than or equal to $N$, and for any open neighborhood $U$ of $z$, some smaller neighborhood $V \subset U$ of $z$ has the following property:

For any diffeomorphism $f \in \operatorname{Diff}^{1}(M)$ that coincides with $f_{0}$ on $U \cup$ $\cdots \cup f_{0}^{N-1}(U)$, for any points $p, q \in M \backslash\left(U \cup \cdots \cup f_{0}^{N}(U)\right)$ and any integers $n_{p}, n_{q} \geq 1$ such that $f^{n_{p}}(p)$ belongs to $V$ and $f^{-n_{q}}(q)$ to $f_{0}^{N}(V)$ there is a diffeomorphism $g \in \operatorname{Diff}^{1}(M)$ arbitrarily close to $f$ which satisfies:

- $g$ coincides with $f$ on $M \backslash\left(U \cup \cdots \cup f_{0}^{N-1}(U)\right)$. The diffeomorphism $g_{0}$ that coincides with $g$ on $U \cup \cdots \cup f_{0}^{N-1}(U)$ and coincides with $f_{0}$ elsewhere belongs to $\mathcal{U}$.

- $g$ sends a positive iterate $g^{m}(p)$ of $p$ on $q$.

- The orbit $\left(p, \cdots, g^{m}(p)\right)$ can be cut in three parts:

a. the beginning $\left(p, \cdots, g^{m^{\prime}}(p)\right)$ has support in

$$
\left\{p, \cdots, f^{n_{p}}(p)\right\} \cup U \cup \cdots \cup f_{0}^{N}(U) ;
$$

b. the central part $\left(g^{m^{\prime}}(p), \cdots, g^{m^{\prime}+N}(p)\right)$ has support in

$$
U \cup \cdots \cup f_{0}^{N}(U)
$$

c. and the end $\left(g^{m^{\prime}+N}(p), \cdots, g^{m}(p)\right)$ has support in

$$
U \cup \cdots \cup f_{0}^{N}(U) \cup\left\{f^{-n_{q}}(q), \cdots, q\right\} .
$$

Obviously, the same statement holds if one shrinks the neighborhood $V$ of $z$.

The main difference with [A] comes from the fact that the integer $N$ is uniform (this is explicit in $[\mathrm{We}]$ ). The statement in $\mathrm{A}_{1}$ also considers only the case $f=f_{0}$ (so that $g=g_{0}$ ). However, the dynamics outside $U \cup \cdots \cup$ $f_{0}^{N-1}(U)$ is not used in the proof. This is the reason why one can replace $f_{0}$ by any map $f$ which coincides with $f_{0}$ on $U \cup \cdots \cup f_{0}^{N-1}(U)$. For a different formulation of the connecting lemma, see also $[\overrightarrow{B Q}$, theorem 2.1]. 


\subsection{Connecting lemma for pseudo-orbits}

In order to handle with the pseudo-orbits, one will use a refinement of Hayashi's connecting lemma which is the connecting lemma for pseudo-orbits proven in $\mathrm{BQ}$.

Theorem 6 (Connecting lemma for pseudo-orbits $B \mathrm{BQ}$ ). - Let $f$ be a diffeomorphism whose periodic orbits are hyperbolic (in particular it satisfies condition $(A))$. Let $\mathcal{U}$ be a neighborhood of $f$ in $\operatorname{Diff}^{1}(M)$.

Then, for any compact set $K \subset M$ and any neighborhood $W$ of $K$, for any points $x$ and $y$ in $K$ such that $x \dashv_{K} y$, there exists a perturbation $g \in \mathcal{U}$ of $f$ whose support is in $W$ and an integer $n \geq 1$ such that $g^{n}(x)=y$. Moreover the points $x, g(x), \ldots, g^{n}(x)$ belong to $W$.

This is proven in $[\mathrm{BQ}$ in the case $K=W=M$ but the proof in the general case is the same: the idea is to cover a large part of $M$ by disjoint "perturbation domains", i.e. by open sets $V$ given by Hayashi's connecting lemma. Let us consider a pseudo-orbit with small jumps and that connects two points $x$ and $y$. The perturbation domains allow to remove the jumps and build an orbit between $x$ and $y$ for a perturbation of $f$.

The diameter of the perturbation domains can be chosen arbitrarily small (in particular, if a perturbation domain intersects the closed set $K$ in theorem 6, it should be contained in $W$ ). Thus, if one considers a pseudoorbit contained in $K$ that connects two points $x, y \in K$ such that $x \dashv_{K} y$, one can use the perturbation domains that intersect $K$ to create a segment of orbit joining $x$ to $y$. Since the perturbation domains used and the pseudoorbits considered are contained in $W$, the support of the perturbation $g$ and the segment of orbit between $x$ and $y$ for $g$ will also be included in $W$ as in theorem 6 .

\subsection{Global connecting lemmas}

We now state the technical perturbation results proven in the paper. Their proofs are based on Hayashi's connecting lemma and are independent from the connecting lemma for pseudo-orbit.

Proposition 8 (Approximation by periodic orbits). - Let $f$ be a diffeomorphism and $\mathcal{U}$ a neighborhood of $f$ in $\operatorname{Diff}^{1}(M)$. Then, there exists an integer $N \geq 1$ with the following property:

If $W \subset M$ is an open set and $X$ a finite set of points in $W$ such that 
- the points $f^{j}(x)$ for $x \in X$ and $j \in\{0, \ldots, N\}$ are pairwise distinct and contained in $W$,

- for any $x, x^{\prime} \in X$, we have $x \prec_{W} x^{\prime}$,

then, for any $\eta>0$ there exist a perturbation $g \in \mathcal{U}$ of $f$ with support in the union of the open sets $f^{j}(\mathrm{~B}(x, \eta))$, for $x \in X$ and $j \in\{0, \ldots, N-1\}$, and a periodic orbit $\mathcal{O}$ of $g$, contained in $W$, which crosses all the balls $\mathrm{B}(x, \eta)$ with $x \in X$.

This proposition gives immediately the theorem 3 stated in the introduction:

Proof of theorem 3. - Let $f$ be a diffeomorphism that satisfies condition $(\mathrm{A}), \mathcal{X}$ be a weakly transitive set of $f$ and $\eta_{0}>0$ a constant. One will assume that $\mathcal{X}$ is not a periodic orbit (otherwise, the conclusion of the theorem is trivially satisfied). We denote by $W$ the $\eta_{0}$-neighborhood of $\mathcal{X}$ in $M$. By proposition 月, since condition (A) is satisfied, there exists some finite set $X \subset \mathcal{X}$, such that every point $z \in \mathcal{X}$ belongs to one ball $\mathrm{B}\left(f^{k}(x), \eta_{0}\right)$ with $x \in X$ and $k \in \mathbf{Z}$. Moreover the orbits of points of $X$ are pairwise disjoint and non-periodic.

Let us consider an arbitrarily small neighborhood $\mathcal{U}$ of $f$ in $\operatorname{Diff}^{1}(M)$ and a constant $\eta \in\left(0, \eta_{0}\right)$ small enough, so that any closed set $K \subset W$, which intersects all the balls $\mathrm{B}(x, \eta)$ with $x \in X$ and which is invariant by a diffeomorphism $g \in \mathcal{U}$, is $\eta_{0}$-close to $\mathcal{X}$ in the Hausdorff topology.

One now applies proposition 8 and one obtains the perturbation $g$ of $f$ and the periodic orbit $\mathcal{O}$ as announced in theorem 3 .

The two next propositions will imply theorem 2 (see section 5.3).

Proposition 9 (Approximation by finite segments of orbits). - Let $f$ be a diffeomorphism and $\mathcal{U}$ a neighborhood of $f$ in $\operatorname{Diff}^{1}(M)$. Then, there exists an integer $N \geq 1$ with the following property:

If $W \subset M$ is an open set and $X=\left\{p_{1}, \ldots, p_{r}\right\}$ a finite set of points in $W$ such that

- the points $f^{j}\left(p_{k}\right)$ for $k \in\{1, \ldots, r\}$ and $j \in\{0, \ldots, N\}$ are pairwise distinct and contained in $W$,

- for any $k, \ell \in\{1, \ldots, r\}$, with $k<\ell$, we have $p_{k} \prec_{W} p_{\ell}$,

then, for any $\eta>0$ there exist a perturbation $g \in \mathcal{U}$ of $f$ with support in the union of the open sets $f^{j}\left(\mathrm{~B}\left(p_{k}, \eta\right)\right)$, for $k \in\{1, \ldots, r\}$ and $j \in\{0, \ldots, N-$ $1\}$, and a finite segment of orbit $\left(p_{1}, g\left(p_{1}\right), \ldots, g^{n}\left(p_{1}\right)\right)$ of $g$, contained in $W$, which crosses all the balls $\mathrm{B}\left(p_{k}, \eta\right)$ with $k \in\{1, \ldots, r\}$. 
Remark. - The segment of orbit built in the proof may visit the points of $X$ in a different order than $p_{1}, p_{2}, \ldots, p_{r}$.

Proposition 10 (Asymptotic approximation). - Let $f$ be a diffeomorphism that satisfies condition $(A)$ and $\mathcal{U}$ a neighborhood of $f$ in $\operatorname{Diff}^{1}(M)$.

We consider:

- an invariant closed set $A$,

- an open neighborhood $\hat{W}_{A}$ of $A$,

- a point $x \in \hat{W}_{A}$ such that $x \prec_{\hat{W}_{A}} A$ (i.e. for every $\varepsilon>0$, there exists a segment of orbit $\left(y, f(y), \ldots, f^{n}(y)\right)$ contained in $\hat{W}_{A}$ which connects $\mathrm{B}(x, \varepsilon)$ to the $\varepsilon$-neighborhood of $A)$.

Then, for any neighborhoods $U$ of $x$ and $W_{A}$ of $A$, there exists a perturbation $g \in \mathcal{U}$ of $f$ and a point $z \in U$ such that:

- the support of the perturbation is contained in $W_{A} \backslash A$ (hence $f$ and $g$ coincide on $A$ );

- the forward orbit of $z$ by $g$ is contained in $\hat{W}_{A}$ and its $\omega$-limit set is contained in $A$.

\subsection{Conservative dynamics}

The perturbation results stated above hold also in different settings, and in particular in the space $\operatorname{Diff}_{v}^{1}(M)$ of $C^{1}$-diffeomorphisms that preserve a volume form $v$, or in the space $\operatorname{Diff}_{\omega}^{1}(M)$ of $C^{1}$-diffeomorphisms that preserve a symplectic form $\omega$. More generally, Pugh and Robinson have given in [PR] some conditions that should be satisfied by a space of diffeomorphisms so that the $C^{1}$-perturbation techniques can be used.

Kupka and Smale's theorem on periodic points of generic diffeomor-

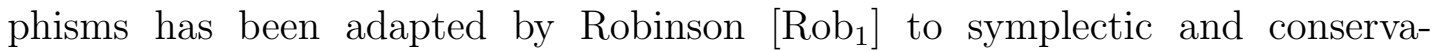
tive diffeomorphisms: in these settings, the periodic orbits of a generic diffeomorphism may be elliptic and not only hyperbolic. In ArBC, we have shown that the connecting lemma for pseudo-orbits remains in $\operatorname{Diff}_{v}^{1}(M)$ and $\operatorname{Diff}_{\omega}(M)$ if one considers any diffeomorphism whose periodic orbits are hyperbolic or elliptic. As a consequence, the generic consequences proven here are also satisfied by the generic conservative or symplectic diffeomorphisms. 


\section{Generic properties of generalized orbits}

We state and prove in this section several generic properties in $\operatorname{Diff}^{1}(M)$. The proofs will use two ingredients:

- the perturbation results (the global perturbations theorems proven in the present paper and the connecting lemma for pseudo-orbits),

- the fact that the set of continuity points of any lower-semi-continuous (or upper-semi-continuous) map $\Gamma: \operatorname{Diff}^{1}(M) \rightarrow \mathcal{K}(\mathcal{K}(M)) \cup\{\emptyset\}$ is a dense $\mathrm{G}_{\delta}$ subset of $\operatorname{Diff}^{1}(M)$.

The $G_{\delta}$ dense subset of diffeomorphisms whose periodic orbits are all hyperbolic will be denoted by $\mathcal{G}_{A}$. In particular, all the diffeomorphisms in $\mathcal{G}_{A}$ satisfy condition (A).

\subsection{Generic comparison of weak and chain-iterates}

For any compact set $K$, the relation $x \prec_{K} y$ implies $x \dashv_{K} y$. The converse is true generically:

Theorem 7 ( $B Q]$ ). — The set of diffeomorphisms such that, for any compact set $K$, the relations $x \prec_{K} y$ and $x \dashv_{K} y$ are equivalent, contains a $G_{\delta}$ dense subset $\mathcal{G}_{\text {chain }}$ of $\operatorname{Diff}^{1}(M)$.

In particular for any diffeomorphism $f \in \mathcal{G}_{\text {chain }}$, we have the equalities

$$
\operatorname{WTrans}(f)=\mathbf{C T r a n s}(f), \quad \mathbf{W O r b}(f)=\mathbf{E O r b}(f), \quad \mathbf{W S e g}(f)=\mathbf{E S e g}(f) .
$$

This theorem is a direct consequence of the connecting lemma for pseudoorbits (theorem 6) and has been proven in $[\mathrm{BC}$ in the case $K=M$. For completeness we give below the proof in the semi-local situation.

Since the relation $\dashv_{K}$ is transitive for any compact set $K$, one deduces the following property. (In the case $K=M$, it was proven by Arnaud $\mathrm{A}_{1}$ and Gan and Wen GW as a consequence of Hayashi's connecting lemma.)

Corollary 2. - For any diffeomorphism $f$ in the $G_{\delta}$ dense subset $\mathcal{G}_{\text {chain }}$ of $\operatorname{Diff}^{1}(M)$, and for any compact set $K$, the relation $\prec_{K}$ is transitive.

Proof of theorem \. - For any diffeomorphism $f$, one denotes by $R_{\prec}(f)$ (resp. by $\left.R_{\dashv}(f)\right)$ the sets of triples $(x, y, K) \in M \times M \times \mathcal{K}(M)$ such that $x \dashv_{K} y$ (resp. $x \prec_{K} y$ ) for the dynamics of $f$. By propositions 1 and 3 , these sets are closed.

Claim 3. - The set $R_{\prec}(f)$ varies lower-semi-continuously with $f$. 
Let us consider a triple $(x, y, K) \in R_{\prec}(f)$. There exists in $R_{\prec}(f)$ a pair of the form $\left(z, f^{n}(z)\right), n \geq 1$, such that $z$ and $f^{n}(z)$ are close to $x$ and $y$ respectively and the segment $\left\{z, f(z), \ldots, f^{n}(z)\right\}$ is contained in a small neighborhood of $K$. One deduces that the compact set $K_{f}=K \cup\left\{z, f(z), \ldots, f^{n}(z)\right\}$ is close to $K$ for the Hausdorff topology. For any small perturbation $g$ of $f$, the set $K_{g}=K \cup\left\{z, g(z), \ldots, g^{n}(z)\right\}$ is close to $K$ in the Hausdorff topology and we have $z \prec_{K_{g}} g^{n}(z)$ for the map $g$. Hence, the triple $\left(z, g^{n}(z), K_{g}\right)$ is close to $(x, y, K)$ in $M \times M \times \mathcal{K}(\mathcal{K}(M))$ and belongs to $R_{\prec}(g)$, showing the lower-semi-continuity of $R_{\prec}(f)$ and the claim.

Consequently, the set of continuity points of $f \mapsto R_{\prec}(f)$ is a dense $\mathrm{G}_{\delta}$ subset $\mathcal{G}_{\prec}$ of $\operatorname{Diff}^{1}(M)$. We set $\mathcal{G}_{\text {chain }}=\mathcal{G}_{A} \cap \mathcal{G}_{\prec}$ where $\mathcal{G}_{A}$ is the set of diffeomorphisms whose periodic orbits are all hyperbolic. We now prove that for any $f \in \mathcal{G}_{\text {chain }}$, we have $R_{\prec}(f)=R_{\dashv}(f)$, which implies the theorem.

Note that we always have the inclusion $R_{\prec}(f) \subset R_{\dashv}(f)$. Let $f$ be a diffeomorphism in $\mathcal{G}_{\text {chain }}, K$ a compact subset of $M$ and $x, y$ two points of $M$ such that $x \dashv_{K} y$ for $f$. Let us also assumes by contradiction that the relation $x \prec_{K} y$ does not hold for $f$. Since $f \in \mathcal{G}_{\prec}$ is a continuity point of the map $g \mapsto R_{\prec}(g)$, there exist some neighborhoods $U, V, W$ of $x, y, K$ in $M$ and a neighborhood $\mathcal{U}$ of $f$ such that: for any diffeomorphism $g \in \mathcal{U}$, there is no segment of orbit $\left\{z, g(z), \ldots, g^{n}(z)\right\}$ with $n \geq 1$, that is contained in $W$ and such that $z$ belongs to $U$ and $g^{n}(z)$ to $V$. However, since $f$ also belongs to $\mathcal{G}_{A}$, one can apply the connecting lemma for pseudo-orbits (theorem 6) and obtain a contradiction.

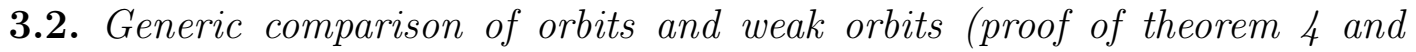
criterion 国)

We will now prove that for $C^{1}$-generic diffeomorphisms, any chain-transitive set is the Hausdorff limit of a sequence of periodic orbits.

Proposition 11. - There exists a $G_{\delta}$ dense subset $\mathcal{G}_{\text {rec }}$ of $\operatorname{Diff}^{1}(M)$ such that for any diffeomorphism $f \in \mathcal{G}_{\text {rec }}$ we have

$$
\operatorname{Per}(f)=\mathbf{W T r a n s}(f)=\mathbf{C T r a n s}(f) .
$$

In particular, we obtain the conclusion of theorem 4 .

Similarly, the extended orbits are approached by finite segments of orbits in the Hausdorff topology:

Proposition 12. - There exists a $G_{\delta}$ dense subset $\mathcal{G}_{\text {orbit }}$ of $\operatorname{Diff}^{1}(M)$ such that for any diffeomorphism $f \in \mathcal{G}_{\text {orbit }}$ we have

$$
\operatorname{Seg}(f)=\mathbf{W} \operatorname{Seg}(f)=\operatorname{ESeg}(f),
$$




$$
\operatorname{FOrb}(f)=\mathbf{W O r b}(f)=\mathbf{E O r b}(f) .
$$

Contrary to the periodic case, we were unable to answer the following problem:

Problem 2. - Does generically the sets $\operatorname{Orb}(f)$ and $\mathbf{F O r b}(f)$ coincide?

A positive answer would imply that $\operatorname{Orb}(f)=\operatorname{EOrb}(f)$ generically in $\operatorname{Diff}^{1}(M)$. Hence, Takens criterion of section 0.3 would be satisfied, proving the tolerance stability conjecture. In other terms, we have shown criterion 2.

The first proposition will be proven by using a result of $\mathrm{F}$. Takens in [T] , which is a consequence of Kupka-Smale genericity theorem [ $\mathbb{K}$, $\left.\mathrm{Sm}_{1}\right]$ : the Per-tolerance stable diffeomorphisms are generic in $\operatorname{Diff}^{1}(M)$.

Theorem 8 (Takens $\left[T_{1}\right]$, theorem II). - The set of continuity points of the map $f \mapsto \operatorname{Per}(f)$ from $\operatorname{Diff}^{1}(M)$ to $\mathcal{K}(\mathcal{K}(M)) \cup\{\emptyset\}$ contains a $G_{\delta}$ dense subset $\mathcal{G}_{\text {Per }}$ of $\operatorname{Diff}^{1}(M)$.

Proof of proposition 11. - Let us define $\mathcal{G}_{\text {rec }}=\mathcal{G}_{A} \cap \mathcal{G}_{\text {Per }} \cap \mathcal{G}_{\text {chain }}$. We consider $f \in \mathcal{G}_{\text {rec }}$. By theorem 7, we have the equality $\mathbf{W T r a n s}(f)=$ CTrans $(f)$. We also have the inclusion $\operatorname{Per}(f) \subset \mathbf{W T r a n s}(f)$ and we will assume by contradiction that the inequalities does not occur: $\operatorname{Per}(f) \neq \mathbf{W} \operatorname{Trans}(f)$. Hence, there exists a weakly transitive invariant compact set $K$ for $f$ which is not accumulated by any periodic orbit. There is a neighborhood $U$ of the point $K \in \mathcal{K}(M)$ such that $\operatorname{Per}(f)$ does not intersect $U$.

By theorem 3, there is a perturbation $g$ of $f$ and a periodic orbit $\mathcal{O}$ of $g$ that is close to $K$ for the Hausdorff topology, and belongs to $U$. This contradicts the fact that $f$ is a continuity point of the map $g \mapsto \operatorname{Per}(g)$. We thus showed that the set $\mathcal{G}_{\text {rec }}$ satisfies the conclusion of proposition 11 .

The second proposition is also proven by using a continuity property. By proposition [4, the map $f \mapsto \mathbf{S e g}(f)$ is lower-semi-continuous, so that the Seg-tolerance stable diffeomorphisms (see the definition of $T$-tolerance stability at section 0.3) are generic in $\operatorname{Diff}^{1}(M)$ :

Proposition 13. - The set of continuity points of the map $f \mapsto \operatorname{Seg}(f)$ from $\operatorname{Diff}^{1}(M)$ to $\mathcal{K}\left(\mathcal{K}(M)\right.$ ) contains a $G_{\delta}$ dense subset $\mathcal{G}_{\text {Seg }}$ of $\operatorname{Diff}^{1}(M)$.

Proof of proposition 19. - We define the residual set $\mathcal{G}_{\text {orbit }}=\mathcal{G}_{A} \cap$ $\mathcal{G}_{\text {Seg }} \cap \mathcal{G}_{\text {chain }}$ and we consider $f \in \mathcal{G}_{\text {orbit }}$. By theorem 7 and since $f$ belongs to $\mathcal{G}_{\text {chain }}$, we have the equality $\mathbf{W S e g}(f)=\mathbf{E S e g}(f)$. We also have the inclusion $\operatorname{Seg}(f) \subset \mathbf{W S e g}(f)$. Let us assume by contradiction that $\operatorname{Seg}(f) \neq$ 
WSeg $(f)$ : there exists a weak segment of orbit $\mathcal{X}$ for $f$ which is not the Hausdorff limit of finite segments of orbits of $f$. Since $f$ is a continuity point of $g \mapsto \operatorname{Seg}(g)$, there is a neighborhood $\mathcal{U}_{0}$ of $f$ and a constant $\eta_{0}>0$ such that the maps $g \in \mathcal{U}_{0}$ have no finite segment of orbit $\eta_{0}$-close to $\mathcal{X}$ for the Hausdorff distance. We denote by $W$ the $\eta_{0}$-neighborhood of $\mathcal{X}$ in $M$.

Claim 4. - There is a finite subset $X=\left\{x_{0}, \ldots, x_{r}\right\}$ of $\mathcal{X}$ such that:

1. $\mathcal{X}$ is $\frac{\eta_{0}}{2}$-close for the Hausdorff distance to the closure of

$$
\left\{f^{n}\left(x_{0}\right)\right\}_{n \geq 0} \cup\left\{f^{-n}\left(x_{r}\right)\right\}_{n \geq 0} \cup\left\{f^{n}\left(x_{k}\right), n \in \mathbf{Z} \text { and } k \in\{1, \ldots, r-1\}\right\} ;
$$

2. $X$ has no periodic point; the orbit of any two distinct points of $X$ are disjoint;

3. for any $k, \ell \in\{0, \ldots, r\}$ with $k<\ell$, we have $x_{k} \prec_{W} x_{\ell}$.

Proof. - Similarly to the proof of proposition 5, one builds using lemma 1 and condition $(\mathrm{A})$ a finite subset $\tilde{X}$ of $\mathcal{X}$ and such that

- $\tilde{X}$ is $\frac{\eta_{0}}{2}$-close to $\mathcal{X}$;

- $\tilde{X}$ has no periodic point.

Since $f$ belongs to $\mathcal{G}_{\text {chain }}$, the relation $\prec \mathcal{X}$ is transitive: one can order the set $\tilde{X}$ as a sequence $\left(\tilde{x}_{0}, \ldots, \tilde{x}_{s}\right)$ such that, for any $k<\ell$ in $\{0, \ldots, s\}$, we have $\tilde{x}_{k} \prec \mathcal{X} \tilde{x}_{\ell}$.

Note that all the points of $\tilde{X}$ can not belong to a same orbit: otherwise $f$ would have a finite segment of orbit contained in $\mathcal{X}$ which is $\frac{\eta_{0}}{2}$-close to $\mathcal{X}$ for the Hausdorff topology, contradicting our assumptions on $\mathcal{X}$ and $\eta_{0}$. By proposition 2, we see that for any $k, \ell \in\{0, \ldots, s\}$, if $\tilde{x}_{k}$ is an iterate of $\tilde{x}_{0}$ and $\tilde{x}_{\ell}$ is not an iterate of $\tilde{x}_{0}$ then, we have $\tilde{x}_{k} \prec \mathcal{X} \tilde{x}_{\ell}$. This shows that, up to reordering the set $X$, one may assume that the iterates of $\tilde{x}_{0}$ contained in $\tilde{X}$ are the first elements of the sequence $\left\{\tilde{x}_{0}, \ldots, \tilde{x}_{s}\right\}$. In particular, the points $\tilde{x}_{0}$ and $\tilde{x}_{s}$ do not belong to a same orbit.

One now removes from $\tilde{X}$ some points and defines a smaller set $X \subset \tilde{X}$ so that $\tilde{x}_{0}$ and $\tilde{x}_{s}$ belongs to $X$, the points of $X$ have different orbits and any point in $\tilde{X}$ has some iterate in $X$. One can order $X$ as a sequence $\left(x_{0}, \ldots, x_{r}\right)$ such that $\tilde{x}_{0}=x_{0}, \tilde{x}_{s}=x_{r}$ and such that, for any $k<\ell$ in $\{0, \ldots, r\}$, we have $x_{k} \prec \mathcal{X} x_{\ell}$. The set $\mathcal{X}$ is not invariant by $f$, but by proposition 2, the whole orbits of the points $x_{1}, \ldots, x_{r-1}$, the positive orbit of $x_{0}$ and the negative orbit of $x_{r}$ are contained in $\mathcal{X}$.

The set $\left\{f^{n}\left(x_{0}\right), n \geq 0\right\} \cup\left\{f^{-n}\left(x_{r}\right), n \geq 0\right\} \cup\left\{f^{n}\left(x_{k}\right), n \in \mathbf{Z}\right.$ and $k \in$ $\{1, \ldots, r-1\}\}$ is contained in $\mathcal{X}$ and contains $\tilde{X}$. Hence, it is $\frac{\eta_{0}}{2}$-close to $\mathcal{X}$ for the Hausdorff distance. 
By the first property of the claim, there exists a neighborhood $\mathcal{U}$ of $f$ contained in $\mathcal{U}_{0}$ and a constant $\eta \in\left(0, \eta_{0}\right)$ such that any finite segment of orbit $\left\{z, g(z), \ldots, g^{n}(z)\right\}$ with $g \in \mathcal{U}$ and $z \in B\left(x_{0}, \eta\right)$ which intersects all the balls $B\left(x_{k}, \eta\right)$ with $x_{k} \in X$, intersects all the balls $B\left(x, \eta_{0}\right)$ with $x \in \mathcal{X}$.

By the two other properties of the claim and theorem 9, there is a perturbation $g$ of $f_{0}$ in $\mathcal{U}$ and a finite segment of orbit $\mathcal{S}=\left\{x_{0}, g\left(x_{0}\right), \ldots, g^{n}\left(x_{0}\right)\right\}$ of $g$ contained in the $\eta_{0}$-neighborhood $W$ of $\mathcal{X}$ which intersects all the balls $B(x, \eta)$ with $x \in X$. In particular, $\mathcal{S}$ is $\eta_{0}$-close to $\mathcal{X}$ for the Hausdorff distance. This contradicts the choice of $\mathcal{U}_{0}$ and $\eta_{0}$, proving the equality $\operatorname{Seg}(f)=$ $\mathbf{W S e g}(f)$ for $f$ in $\mathcal{G}_{\text {orbit }}$.

Since the elements of $\mathbf{F O r b}(f)$ and $\mathbf{W O r b}(f)$ are the elements of $\operatorname{Seg}(f)$ and $\mathbf{W S e g}(f)$ respectively that are invariant by $f$, one also gets the equality

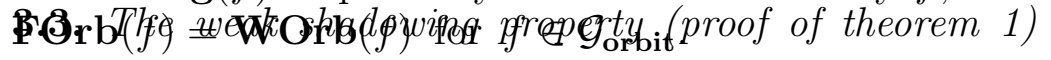

For any $\varepsilon>0$, we consider the set of finite segments of $\varepsilon$-pseudoorbits. Taking the closure in $\mathcal{K}(M)$ we get a set $\mathbf{E S e g}_{\varepsilon}(f)$. The family $\left(\mathbf{E S e g}_{\varepsilon}(f)\right)_{\varepsilon>0}$ is ordered for the inclusion and the intersection $\cap_{\varepsilon} \mathbf{E S e g}_{\varepsilon}(f)$ coincides with $\operatorname{ESeg}(f)$.

For any diffeomorphism $f$ in the residual set $\mathcal{G}_{\text {Orb }}$ given by propositions 11 and 12 and any $\delta>0$ we consider $\varepsilon>0$ such that the Hausdorff distance in $\mathcal{K}(\mathcal{K}(M))$ between $\mathbf{E S e g}(f)$ and $\mathbf{E S e g}_{\varepsilon}(f)$ is less than $\frac{\delta}{2}$.

Let $\left\{z_{0}, \ldots, z_{n}\right\}$ be any $\varepsilon$-pseudo-orbit of $f$. It belongs to $\mathbf{E S e g}_{\varepsilon}(f)$, hence there exists an element $K \in \mathbf{E S e g}(f)$ such that the Hausdorff distance between $K$ and $\left\{z_{0}, \ldots, z_{n}\right\}$ in $\mathcal{K}(M)$ is less than $\frac{\delta}{2}$. By proposition 12, the set $K$ also belongs to $\operatorname{Seg}(f)$ so that there exists a finite segment of orbit $\left\{x, f(x), \ldots, f^{m}(x)\right\}$ which is $\frac{\delta}{2}$-close to $K$. This shows that $\left\{z_{0}, \ldots, z_{n}\right\}$ and $\left\{x, \ldots, f^{m}(x)\right\}$ are $\delta$-close for the Hausdorff distance.

One can consider similarly for each $\varepsilon>0$ the set of periodic $\varepsilon$-pseudoorbits $\left\{z_{0}, \ldots, z_{n}\right\}$ with $n \geq 1$ and $z_{n}=z_{0}$. Their closure in $\mathcal{K}(M)$ defines the set $\operatorname{CTrans}_{\varepsilon}(f)$. By proposition 11, one can make a similar argument as above with the sets $\mathcal{G}_{\text {rec }},\left(\mathbf{C T r a n s}_{\varepsilon}(f)\right)_{\varepsilon>0}, \mathbf{C T r a n s}(f)$ and $\operatorname{Per}(f)$ instead of $\mathcal{G}_{\text {Orb }},\left(\operatorname{ESeg}_{\varepsilon}(f)\right)_{\varepsilon>0}, \operatorname{ESeg}(f)$ and $\operatorname{Seg}(f)$ to get the second part of theorem 1 .

Hence, theorem 11 holds with the $\mathrm{G}_{\delta}$ and dense subset $\mathcal{G}_{\text {shadow }}=\mathcal{G}_{\text {rec }} \cap$ $\mathcal{G}_{\text {orbit }}$ of $\operatorname{Diff}^{1}(M)$. 


\subsection{Tolerance stability properties}

We were unable to decide if the continuity points of the map $f \mapsto$ $\operatorname{Orb}(f)$ are generic in $\operatorname{Diff}^{1}(M)$ (which is the original Zeeman's tolerance stability conjecture) but we get the property for all the other sets introduced in the paper:

Proposition 14. - There is a residual subset of $\operatorname{Diff}^{1}(M)$ whose elements are Per-, FOrb-, Seg-, CTrans-, EOrb-, ESeg-, WTrans-, WOrb- and WSeg-tolerance stable.

Proof. - For Per, this was proven by Takens (see theorem 8 above), the case of FOrb and Seg comes from the fact they depend lower-semicontinuously in the dynamics (proposition 4) and the case of CTrans, EOrb, ESeg from the fact that they vary upper-semi-continuously with the dynamics (proposition 3).

The remaining sets: WTrans, WOrb and WSeg are always controlled by the other ones (using the inclusions (11), (2), (3)). Since generically the inclusions (11), (21), (3) are equalities and since we have already proven the proposition for the six first sets, one deduces the proposition for the last ones.

\subsection{Approximations of aperiodic classes (proof of corollary [)}

Let $f$ be a diffeomorphism in $\mathcal{G}_{\mathrm{A}} \cap \mathcal{G}_{\text {rec }}$ and let $E$ be a aperiodic class of $f$. In order to prove corollary 11 we show that $E$ is the limit of a sequence of homoclinic classes for the Hausdorff topology. We fix some $\varepsilon>0$.

We recall an upper-semi-continuity property of the chain-recurrence classes.

Lemma 2. - There exists a neighborhood $U$ of $E$ such that any chainrecurrence class $E^{\prime}$ of $f$ which intersects $U$ is contained in the $\varepsilon$-neighborhood of $E$.

Proof. - One proves the lemma by contradiction. Let $\left(E_{n}\right)$ be a sequence of chain-recurrence classes which converges (for the Hausdorff topology) towards a compact set $K$, that intersects $E$ but is not contained in $E$. By proposition 3, $K$ is chain-transitive and contained in a chain-recurrence class. But $K$ intersects $E$ and the chain-recurrence classes are disjoint or equal. So, $K$ is contained in $E$, which is a contradiction.

By proposition 11, there exists a periodic orbit $\mathcal{O}$ of $f \in \mathcal{G}_{\text {rec }}$, which is close to $E$ in the Hausdorff topology: $\mathcal{O}$ is contained in $U$ and $E$ is contained 
in the $\varepsilon$-neighborhood of $\mathcal{O}$. Since $f$ belongs to $\mathcal{G}_{\mathrm{A}}$, the periodic orbit $\mathcal{O}$ is hyperbolic.

The chain-recurrence class $E^{\prime}$ which contains $\mathcal{O}$ intersects $U$ and, by lemma 2, is contained in the $\varepsilon$-neighborhood of $E$. The homoclinic class $H$ which contains $\mathcal{O}$ is included in $E^{\prime}$, hence in the $\varepsilon$-neighborhood of $E$. Since $H$ contains $\mathcal{O}$, we also get that the $\varepsilon$-neighborhood of $H$ contains $E$. We thus found a homoclinic class $H$ of $f$ which is $\varepsilon$-close to $E$ for the Hausdorff distance. Consequently, $E$ is accumulated by homoclinic classes.

We proved corollary [1] with $\mathcal{G}_{\text {aper }}=\mathcal{G}_{\mathrm{A}} \cap \mathcal{G}_{\text {rec }}$.

\section{Approximation of weakly transitive sets by periodic orbits: proof of proposition 8}

In this part, one considers a diffeomorphism $f$ and a neighborhood $\mathcal{U}$ of $f$ in $\operatorname{Diff}^{1}(M)$. By shrinking $\mathcal{U}$ if necessary, one may assume that it satisfies the condition $(\mathrm{F})$ : for any perturbations $g_{1}, g_{2} \in \mathcal{U}$ of $f$ with disjoint support, the composed perturbation also belongs to $\mathcal{U}$.

The connecting lemma (theorem 5) associates to $f$ and $\mathcal{U}$ some integer $N \geq 1$ that will be fixed from now on and used all along this part.

In order to prove proposition 8 , we consider in $M$ a finite set $X$ and an open set $W$ that verify the assumptions of the proposition. We also choose a constant $\eta>0$. All the segments of orbits we will consider in the following will be contained in $W$.

\subsection{Sketch of the proof of proposition 8}

The main ingredient of the proof is Hayashi's connecting lemma. The natural idea is the following: one cyclically orders the points of $X=\left\{p_{1}, p_{2}, \ldots, p_{r}\right\}$. At each of these points $p_{i}$, one can apply the connecting lemma and associate two neighborhoods $V_{i} \subset U_{i}$ (contained in $W$ ): these neighborhoods may be chosen contained in arbitrarily small balls $B\left(p_{i}, \varepsilon_{2}\right)$ and $B\left(p_{i}, \varepsilon_{1}\right)$ respectively, centered at $p_{i}$ and of radii $0<\varepsilon_{2}<\varepsilon_{1}$. Using the definition of weak iterate, one chooses for each $i$ a segment of orbit $Z_{i}$ (contained in $W$ ) that connects $V_{i}$ to $V_{i+1}$ (where the subscripts are taken modulo $k$ ) and then tries to use the connecting lemma to connect all the $Z_{i}$ together by perturbing $f$ in each of the domains $U_{i}$ and their $N-1$ first iterates: the composed perturbation $g$ belongs to $\mathcal{U}$ thanks to the condition $(\mathrm{F})$; moreover, if the domains $U_{i}$ have been chosen small enough, one would get a periodic orbit that intersects all the balls $B\left(p_{i}, \eta\right)$ and that is contained in $W$. 
Several difficulties arise in this naive approach. A first problem appears when a segment of orbit $Z_{i}$ intersects a neighborhood $U_{j}$ of index $j$ different from $i$ and $i+1$ : the perturbation with support in $U_{j}$ may break the segment $Z_{i}$ and we are not sure anymore to get a periodic orbit for the composed perturbation $g$. To over-pass this problem, one notes that in this case the segment of orbit $Z_{i}$, that connects the neighborhoods of $p_{i}$ and $p_{i+1}$ together, also intersects the neighborhood of $p_{j}$ : it may not be necessary to consider the segments $Z_{j-1}, Z_{j}$ and to perturb $f$ around $p_{j}$ in order to create a periodic orbit with the required support. More precisely, one will choose a smaller set $X^{\prime} \subset X$ of points where the connecting lemma will be applied. One orders it as $X^{\prime}=\left\{x_{0}, \ldots, x_{s}\right\}$ and one chooses the neighborhoods $V_{i} \subset U_{i}$ at each point $x_{i}$ and then the segments of orbits $Z_{i}$ connecting $U_{i}$ to $U_{i+1}$. As explained above, each segment $Z_{i}$ may intersect a small neighborhood of several points in $X$ (not only $x_{i}$ and $x_{i+1}$ ): the set of points that are "visited" by $Z_{i}$ will be denoted by $X_{i, i+1}$. Although $X^{\prime}$ is smaller than $X$, one can hope to create a periodic orbit that visit all the points of $X$ if we have

$$
X=X^{\prime} \cup X_{0,1} \cup \cdots \cup X_{s-1, s} \cup X_{s, 0} .
$$

In order to prevent any segment of orbit $Z_{j}$ to intersect the domains $U_{j}$ with $j \neq i, i+1$, one will choose $X^{\prime}$ with the smallest possible cardinality $s$.

We now meet a second difficulty: when one applies the connecting lemma in a domain $U_{i}$ to the segments of orbits $Z_{i-1}$ and $Z_{i}$, one gets a new segment of orbit that connects $U_{i-1}$ to $U_{i+1}$, and that is contained in $Z_{i-1} \cup$ $\left(\bigcup_{0 \leq k \leq N} f^{k}\left(U_{i}\right)\right) \cup Z_{i}$. However, it should be noted that the connecting lemma can realize some shortcuts: some parts of the orbits $Z_{i-1}$ and $Z_{i}$ may be forgotten; hence, the new segment of orbit may not visit the neighborhood of some of the points of $X_{i-1, i} \cup X_{i, i+1}$. As a consequence, the periodic orbit that one builds may miss some of the points of $X$, even if the condition (4) is satisfied. To avoid this difficulty, one requires that among all the segments of orbits that connect $U_{i}$ to $U_{i+1}$, the orbit $Z_{i}$ minimizes the cardinality of the set $X_{i, i+1}$ of points it visits. By doing that, any shorter segment of orbit will visit exactly the same set $X_{i, i+1}$.

We now give the plan of the proof:

First step (section 4.1.). The construction of the minimizing sets $X_{i, i+1}$ is done before choosing the orbits $Z_{i}$, and the set $X^{\prime}$ : more precisely, one introduce a minimizing visit set $X_{x, x^{\prime}}$ for each pair $x \neq x^{\prime}$ in $X$.

Second step (section 4.9 ). One then chooses the set $X^{\prime}=\left\{x_{0}, \ldots, x_{s}\right\}$. In the best case, each set $X_{i, i+1}$ does not contains any point $x_{j} \in X^{\prime}$ with $j \neq i, i+1$, so that $Z_{i}$ and $U_{j}$ will be disjoint. In general, it is not possible to have this property, but we show (proposition 16) that one can always 
have half of it: for each $i, j \in\{0, \ldots, s\}$ such that $i+1<j$, the point $x_{j}$ does not belong to $X_{i, i+1}$.

Third step (section 4.3). From the second step, we have to ensure that for each $j<i<s$, the neighborhood $U_{j}$ does not intersect the segment of orbit $Z_{i}$. This is done in proposition 17 where one builds inductively the neighborhoods $V_{i} \subset U_{i}$ and the segments of orbits $Z_{i}$. One starts with the largest indices $i=s, s-1, \ldots, 0$.

Fourth step (section 4.4. ). It now remains to perturb in each domain $U_{i}$ in order to connect all the $Z_{i}$. One obtains a segment of orbit that visits all the points of the set $X$.

However, the most delicate part of the proof consists in proving that this segment can be closed and that one can obtain a periodic orbit:

- As explained, the segment of orbit $Z_{s}$ should be chosen at the beginning of the third step, so that, the domains $U_{j}$, with $j \in\{1, \ldots, s-1\}$ can be build disjoint from $Z_{s}$. One problem is the following: $Z_{s}$ should connect $V_{s}$ to the open set $V_{0}$ but $V_{0}$ will be constructed only at the end of step 3... This paradox is solved by noting (corollary 4) that the set $Y \subset\left\{x_{0}\right\} \cup X_{0,1}$ of points in $X$ that are not contained in $X_{1,2} \cup \cdots \cup X_{s-1, s}$ is non-empty. In the simplest case $x_{0}$ belongs to $Y$. Hence, if one chooses a priori the neighborhoods $V_{0}$ and $U_{0}$ equal to $B\left(x_{0}, \varepsilon_{2}\right)$ and $B\left(x_{0}, \varepsilon_{1}\right)$, they will be disjoint from the orbits $Z_{1}, \ldots, Z_{s-1}$; so, without breaking the orbit at intermediate iterates, one can close the orbit by perturbing in $U_{0}$.

- In general, this set $Y$ may not contain the point $x_{0}$. One has to choose a new point $x_{s+1}$ in $Y$ where the orbit will be closed: the choice of this point is not given a priori, but will depend on the construction of all the segments $Z_{1}, \ldots, Z_{s-1}$. For this reason, at the beginning of the construction, one has to consider all the possible choices for $x_{s+1}$ : for each point $y \in Y$, one defines two neighborhoods $V_{y} \subset U_{y}$ and one introduces a segment of orbit $Z_{y}$ that connects $V_{s}$ to $V_{y}$. This allows in the following to take the other neighborhoods $U_{j}$ disjoint from all the $Z_{y}$. At the end, one chooses $x_{s+1}$ in $Y$ and one defines $Z_{s}=Z_{x_{s+1}}, V_{s+1}=V_{x_{s+1}}$ and $U_{s+1}=U_{x_{s+1}}$. A perturbation in $U_{s+1}$ will connect the orbit $Z_{s+1}$ to the orbit $Z_{0}$ and close the orbit.

- Note that we have to ensure that the orbit $Z_{0}$ crosses the neighborhood $V_{s+1}$ in order to perform this last perturbation. By our construction, the neighborhoods $V_{s+1}$ and $U_{s+1}$ can be chosen a priori equal to the balls $B\left(x_{s+1}, \varepsilon_{2}\right)$ and $B\left(x_{s+1}, \varepsilon_{1}\right)$ respectively. In other terms, the points of $X_{0,1}$ should be at distance less than $\varepsilon_{2}$ from $Z_{0}$. This was not the case for the other segments of orbits $Z_{j}$, with $j \in\{1, \ldots, s-1\}$ : the points in $X_{j, j+1}$ 
are at distance less than $\eta$ from $Z_{j}$. This shows that several scales should be considered in the proof:

- In order to obtain the minimizing property of the sets $X_{i, i+1}$, the (largest) scale $\eta$ given in the statement of the proposition will be replaced by a smaller constant $\varepsilon_{0}>0$.

- The (smaller) scales $\varepsilon_{2}<\varepsilon_{1}$ give the size of the domains $V_{y} \subset U_{y}$ chosen a priori for the connecting lemma at points of $Y$.

- One has also to control the points that are visited at scale $\varepsilon_{2}$ by the segment $Z_{0}$. For this purpose, the endpoints of $Z_{0}$ will be taken close to $x_{0}$ and $x_{1}$ (see section 4.1): the scale here is $\varepsilon_{3} \in\left(0, \varepsilon_{2}\right)$.

- The choice of the point $x_{s+1}$ is the remaining difficulty. The last perturbation connects $Z_{s}$ to $Z_{0}$ in $U_{s+1}$ which is in general different from $U_{0}$. For this reason, the minimizing properties of the set $X_{0,1}$ can not be used here to guarantee that after perturbation, the orbit visit all the points of $X_{0,1}$ : in particular, when one perturbs in $U_{1}$, the connecting lemma shortcuts the segment of orbit (contained in $Z_{0}$ ) between $U_{s+1}$ and $U_{1}$, so that some points in $X_{0,1}$ may be missed. This is why at section 4.4 the perturbation in $U_{1}$ will be performed before choosing the point $x_{s+1}$.

\subsection{Visited sets}

In this section, we give several definitions that will be used in the following proofs. In particular, for any points $x \neq x^{\prime}$ in the set $X$ given above, we introduce a subset $X_{x, x^{\prime}} \subset X$ : it contains the points in $X$ that, in some sense, can not be avoided by the orbits which come close to $x$ and then to $x^{\prime}$.

We will study the orbits that approach $X$ at different scales $\varepsilon \in\left(0, \varepsilon_{0}\right]$ where $\varepsilon_{0}$ is a small constant in $(0, \eta)$ that will be chosen at proposition 15 . As we have explained in 4.0, four different scales $\varepsilon_{0}>\varepsilon_{1}>\varepsilon_{2}>\varepsilon_{3}$ will be needed in the proof. Recall also that the integer $N \geq 1$ has been fixed above by Hayashi's connecting lemma.

Definition 6. - Let $x, x^{\prime}$ be two distinct points in $X$ and $U, U^{\prime}$ two neighborhoods of $x$ and $x^{\prime}$.

A finite segment of orbit $\left(z, f(z), \ldots, f^{n}(z)\right)$ contained in $W$ connects $U$ to $U^{\prime}$ if $z \in f^{N}(U)$ and $f^{n}(z) \in U^{\prime}$.

Such a segment of orbit $\varepsilon$-visits some $y \in X$ if some iterate $f^{k}(z)$ with $k \in\{0, \ldots, n\}$ belongs to $\mathrm{B}(y, \varepsilon)$. We denote by $X\left(z, \cdots, f^{n}(z), \varepsilon\right) \subset X$ the set of points which are $\varepsilon$-visited by the segment of orbit $\left(z, \ldots, f^{n}(z)\right)$ and by $N\left(z, \cdots, f^{n}(z), \varepsilon\right)$ the cardinality of this set. 
The segment of orbit is said to $\varepsilon$-visit (resp. $\varepsilon$-visit exactly) some set $Y \subset X$ if $Y \subset X\left(z, \cdots, f^{n}(z), \varepsilon\right) \quad\left(\operatorname{resp} . Y=X\left(z, \cdots, f^{n}(z), \varepsilon\right)\right)$.

Note that the assumptions made in proposition 8 on the set $X$ ensure that for any choice of $U$ and $U^{\prime}$, there always exists a segment of orbit connecting these neighborhoods.

We now introduce the minimizing sets $X_{x, x^{\prime}}$. There is some freedom in the construction and their choice is not unique, but will be fixed in the following sections.

Proposition 15. - There exist a constant $\varepsilon_{0} \in(0, \eta)$, and for each distinct points $x, x^{\prime}$ in $X$, an integer $N\left(x, x^{\prime}\right)$ and a set $X_{x, x^{\prime}} \subset X$ of cardinality $N\left(x, x^{\prime}\right)$ having the following property.

For any $\varepsilon \in\left(0, \varepsilon_{0}\right)$, and any distinct points $x, x^{\prime} \in X$, there are two neighborhoods $U\left(x, x^{\prime}, \varepsilon\right)$ and $U^{\prime}\left(x, x^{\prime}, \varepsilon\right)$ of $x$ and $x^{\prime}$ respectively such that:

- any segment of orbit $\left(z, f(z), \ldots, f^{n}(z)\right)$ that connects $U\left(x, x^{\prime}, \varepsilon\right)$ to $U^{\prime}\left(x, x^{\prime}, \varepsilon\right) \varepsilon$-visits at least $N\left(x, x^{\prime}\right)$ points in $X$;

- for any neighborhoods $U \subset U\left(x, x^{\prime}, \varepsilon\right)$ and $U^{\prime} \subset U^{\prime}\left(x, x^{\prime}, \varepsilon\right)$ of $x$ and $x^{\prime}$, there exists a finite segment of orbit that connects $U$ to $U^{\prime}$, that is disjoint from $X$ and that $\varepsilon$-visits exactly the set $X_{x, x^{\prime}}$.

Proof. - Let us choose two distinct points $x, x^{\prime} \in X$, some neighborhoods $U, U^{\prime}$ of $x$ and $x^{\prime}$ and a constant $\varepsilon \in(0, \eta)$. We consider $N\left(U, U^{\prime}, \varepsilon\right)$ the minimum of $N\left(z, \cdots, f^{n}(z), \varepsilon\right)$ over all the orbits $\left(z, \cdots, f^{n}(z)\right)$ that connect $U$ to $U^{\prime}$. Taking $U$ or $U^{\prime}$ smaller, this number increases, but is bounded by the cardinality of $X$. Thus, there exists some maximal number $N\left(x, x^{\prime}, \varepsilon\right)$ such that for any $U$ and $U^{\prime}$ small enough, $N\left(x, x^{\prime}, \varepsilon\right)$ is equal to $N\left(U, U^{\prime}, \varepsilon\right)$.

Note that one can assume that all the segments of orbits we used to connect $U$ to $U^{\prime}$ are disjoint from $X$ : the first point $z$ may be replaced by any other point $\tilde{z}$ in a small neighborhood of $z$; the new segment of orbit $\left(\tilde{z}, \cdots, f^{n}(\tilde{z})\right)$, with the same length, will connect again $U$ to $U^{\prime}$ and $\varepsilon$-visit the same set. As $X$ is finite, one can adjust $\tilde{z}$ in order that its orbit avoids $X$.

As $\varepsilon>0$ decreases, the number $N\left(x, x^{\prime}, \varepsilon\right)$ decreases also. The minimum of $N\left(x, x^{\prime}, \varepsilon\right)$ over all $\varepsilon$ will be denoted by $N\left(x, x^{\prime}\right)$. We choose now the constant $\varepsilon_{0} \in(0, \eta)$ small enough so that for any $x$ and $x^{\prime}$ in $X$, and any $\varepsilon \in\left(0, \varepsilon_{0}\right]$ we have $N\left(x, x^{\prime}, \varepsilon\right)=N\left(x, x^{\prime}\right)$.

As $X$ is finite, there are only finitely many possible sets $X\left(z, \cdots, f^{n}(z), \varepsilon\right)$ with cardinality $N\left(x, x^{\prime}\right)$. Hence, there exists $X_{x, x^{\prime}} \subset X$ with cardinality $N\left(x, x^{\prime}\right)$ such that for any $\varepsilon \in\left(0, \varepsilon_{0}\right)$ and any neighborhoods $U$ and $U^{\prime}$ of $x$ and $x^{\prime}$, some orbit connects $U$ to $U^{\prime}$ and $\varepsilon$-visits exactly the set $X_{x, x^{\prime}}$. 
For any $\varepsilon \in\left(0, \varepsilon_{0}\right)$, the neighborhoods $U\left(x, x^{\prime}, \varepsilon\right)$ and $U^{\prime}\left(x, x^{\prime}, \varepsilon\right)$ of $x$ and $x^{\prime}$ are chosen such that $N\left(U\left(x, x^{\prime}, \varepsilon\right), U^{\prime}\left(x, x^{\prime}, \varepsilon\right)\right)=N\left(x, x^{\prime}\right)$.

In order to justify our choice of minimizing sets $X_{x, x^{\prime}}$, we state the following corollary.

Corollary 3. - Let us consider any $\varepsilon \in\left(0, \varepsilon_{0}\right]$, any $x, x^{\prime} \in X$, any small neighborhoods $U \subset U\left(x, x^{\prime}, \varepsilon\right), U^{\prime} \subset U^{\prime}\left(x, x^{\prime}, \varepsilon\right)$ of $x$ and $x^{\prime}$, any segment of orbit $Z=\left(z, \cdots, f^{n}(z)\right)$ that connects $U$ to $U^{\prime}$ and $\varepsilon$-visits exactly the set $X_{x, x^{\prime}}$. Then, any sub-orbit $Z^{\prime}$ of $Z$, that connects $U\left(x, x^{\prime}, \varepsilon\right)$ to $U^{\prime}\left(x, x^{\prime}, \varepsilon\right)$, $\varepsilon$-visits exactly the set $X_{x, x^{\prime}}$.

Proof. - The set $\Xi$ of points that are $\varepsilon$-visited by $Z^{\prime}$ has cardinality at least $N\left(x, x^{\prime}\right)$ by definition. On the other side $\Xi$ is contained in the set $X_{x, x^{\prime}}$ of points that are $\varepsilon$-visited by $Z$. This concludes the proof since $X_{x, x^{\prime}}$ has cardinality $N\left(x, x^{\prime}\right)$.

Choice of the scales. - By having chosen the constant $\varepsilon_{0}$ small enough, one may first assume that:

a. All the balls $f^{k}\left(\mathrm{~B}\left(x, \varepsilon_{0}\right)\right)$ with $x \in X$ and $k \in\{0, \ldots, N\}$ are pairwise disjoint.

We will use later four different scales $0<\varepsilon_{3}<\varepsilon_{2}<\varepsilon_{1}<\varepsilon_{0}$ for the balls visited by the connecting orbits. We choose them inductively: let us assume that $\varepsilon_{i}$ has been defined. For each $x \in X$, the connecting lemma at $x$ associates to $U_{x}=\mathrm{B}\left(x, \varepsilon_{i}\right)$ a smaller neighborhood $V_{x}$ of $x$. We choose $0<\varepsilon_{i+1}<\varepsilon_{i}$ so that for each $x \in X$, the ball $\mathrm{B}\left(x, \varepsilon_{i+1}\right)$ is contained in $V_{x}$ and in both sets $U\left(x, x^{\prime}, \varepsilon_{i}\right)$ and $U^{\prime}\left(x^{\prime}, x, \varepsilon_{i}\right)$ for each $x^{\prime} \in X \backslash\{x\}$.

From this choice, the following additional properties are satisfied:

b. For every $x \in X$, the connecting lemma may be applied at $x$ with the time $N$ and the neighborhoods $\mathrm{B}\left(x, \varepsilon_{i+1}\right) \subset \mathrm{B}\left(x, \varepsilon_{i}\right)$.

c. For every $x, x^{\prime} \in X$ and any neighborhoods $U \subset B\left(x, \varepsilon_{i+1}\right), U^{\prime} \subset$ $B^{\prime}\left(x^{\prime}, \varepsilon_{i+1}\right)$, there exists a finite segment of orbit that connects $U$ to $U^{\prime}$, that is disjoint from $X$, and that $\varepsilon_{i+1^{-}}$-visits exactly the set $X_{x, x^{\prime}}$.

\subsection{Combinatorics of $X$}

We choose here the set $X^{\prime} \subset X$ of points where the connecting lemma will be applied. 
Proposition 16. - There exists a sequence $X^{\prime}=\left\{x_{0}, \cdots, x_{s}\right\} \quad(s \geq 1)$ of distinct points of $X$ such that the visited sets $X_{k, k+1}=X_{x_{k}, x_{k+1}}$ satisfy the following properties:

1. $X=\left\{x_{0}, \cdots, x_{s}\right\} \cup X_{0,1} \cup \cdots \cup X_{s-1, s}$.

2. For any $2 \leq k \leq s$, the point $x_{k}$ does not belong to

$$
\left\{x_{0}, x_{1}, \cdots, x_{k-1}\right\} \cup X_{0,1} \cup X_{1,2} \cup \cdots \cup X_{k-2, k-1} \text {. }
$$

Proof. - One first chooses arbitrarily some point $x_{0} \in X$. Each time the point $x_{k}$ (for $k \geq 0$ ) has been defined one considers inductively any point $x_{k+1}$ in $X \backslash\left(\left\{x_{0}, \cdots, x_{k}\right\} \cup X_{0,1} \cup \cdots \cup X_{k-1, k}\right)$ unless this last set covers already all $X$ (this means $k=s$ ).

In proposition 16, the set $X^{\prime}$ is not unique. The next corollary shows that some choices are better.

Corollary 4. - If the set $X^{\prime}$ in proposition 10 has the smallest cardinality $s$, then, the set

$$
Y=\left(\left\{x_{0}\right\} \cup X_{0,1}\right) \backslash\left(\left\{x_{1}, \ldots, x_{s}\right\} \cup X_{1,2} \cup \cdots \cup X_{s-1, s}\right)
$$

is non-empty.

Proof. - Let us suppose that the corollary is not satisfied: the set $\left\{x_{1}, \cdots, x_{s}\right\} \cup X_{1,2} \cup \cdots \cup X_{s-1, s}$ contains all points in $X$. Thus, the smaller sequence $\left(x_{1}, \cdots, x_{s}\right)$ also satisfies properties (1) and (2) of proposition 16, which is a contradiction since $s$ is minimal.

Notation. - We will denote the set $X_{k, k+1}$ by $X_{x_{k}, x_{k+1}}$ and $X_{x_{s}, y}$ by $X_{s, y}$ for any $k \in\{0, \ldots, s-1\}$ and $y \in Y$.

\subsection{Choosing orbits and neighborhoods}

We explain in the next proposition how to choose inductively, at each point in the sets $X^{\prime}, Y \subset X$, a pair of neighborhoods $V \subset U$ where the connecting lemma may be applied and some segments of orbits that connects these neighborhoods (see figure 2).

Proposition 17. - Let $X^{\prime}=\left\{x_{0}, \ldots, x_{s}\right\}$ and $Y$ be the subsets of $X$ given by proposition 10 and corollary 6 . Then, there exists

- a family of open sets $U_{k} \subset \mathrm{B}\left(x_{k}, \varepsilon_{2}\right)$ for each $k \in\{0, \ldots, s\}$ and $U_{y}=$ $\mathrm{B}\left(y, \varepsilon_{1}\right)$ for each $y \in Y$, 
- a family of smaller open sets $V_{k} \subset U_{k}$ for each $k \in\{0, \ldots, s\}$ and $V_{y}=\mathrm{B}\left(y, \varepsilon_{2}\right)$ for each $y \in Y$,

- a segment of orbit $Z_{k}$ that connects $V_{k}$ to $V_{k+1}$ for each $k \in\{0, \ldots, s-$ $1\}$,

- a segment of orbit $Z_{y}$ that connects $V_{s}$ to $V_{y}$ for each $y \in Y$,

such that the following properties are satisfied:

1. The open set $V_{0}$ is contained in $\mathrm{B}\left(x_{0}, \varepsilon_{3}\right)$ and $U_{1}$ in $\mathrm{B}\left(x_{1}, \varepsilon_{3}\right)$.

2. The connecting lemma may be applied to the neighborhoods $V_{k} \subset U_{k}$ and to the neighborhoods $V_{y} \subset U_{y}$ with the time $N$.

3. For any $y \in Y$, the orbit $Z_{y}$ does not intersect the sets $U_{\ell}$ with $\ell \in$ $\{1, \ldots, s-1\}$; moreover, it $\varepsilon_{0}$-visits exactly the set $X_{s, y}$.

4. For any $k \in\{1, \ldots, s-1\}$, the orbit $Z_{k}$ does not intersect the sets $U_{\ell}$ with $\ell \neq k, k+1$ nor the $U_{y}$ for $y \in Y$; moreover, it $\varepsilon_{0}$-visits exactly the set $X_{k, k+1}$.

5. The orbit $Z_{0}$ does not intersect the sets $U_{k}$ with $k \in\{2, \ldots, s\}$; moreover, it $\varepsilon_{2}$-visits exactly the set $X_{0,1}$.

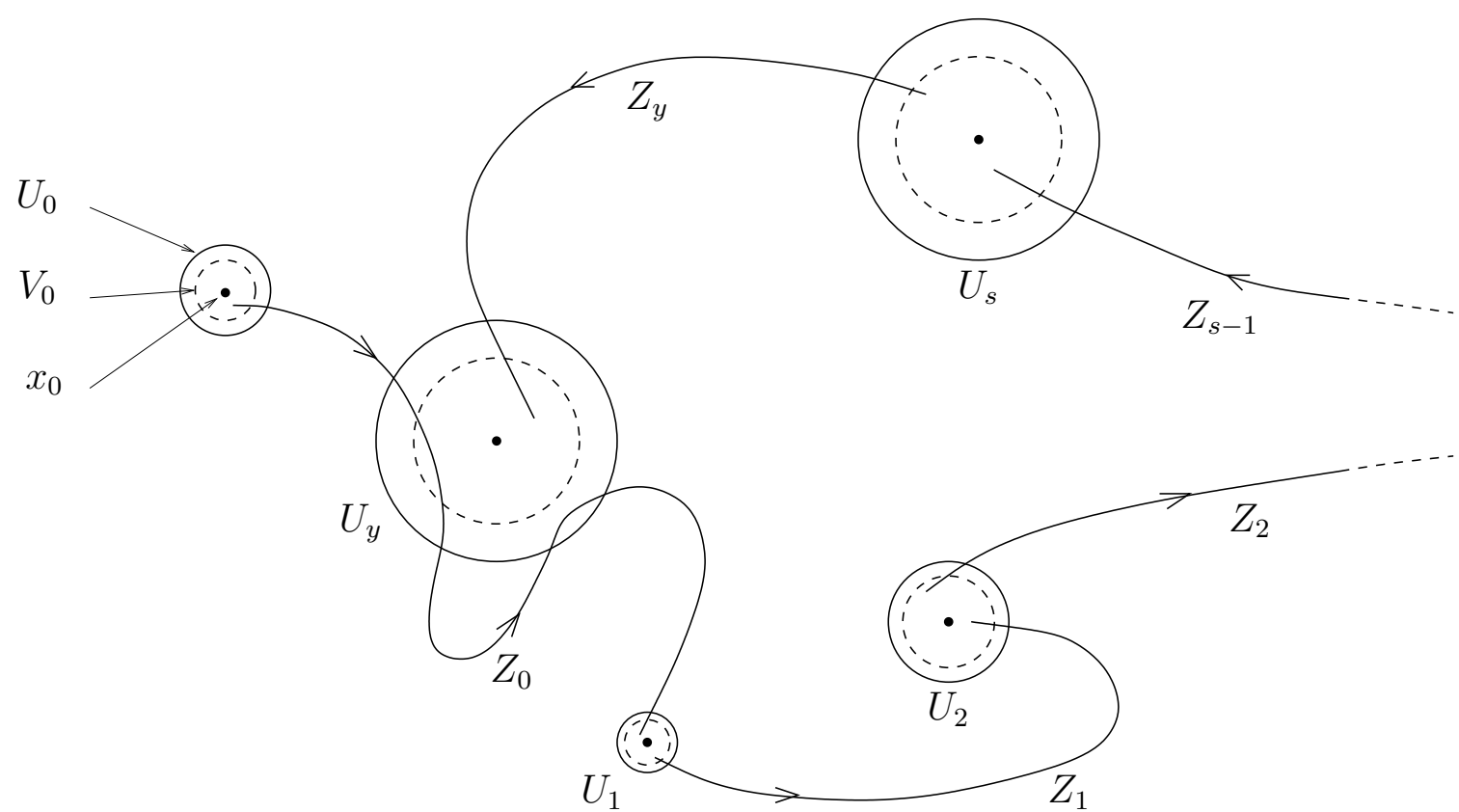

FIG. 2. - 
Proof. - We first set for any $y \in Y$,

$$
U_{y}=\mathrm{B}\left(y, \varepsilon_{1}\right), \quad V_{y}=\mathrm{B}\left(y, \varepsilon_{2}\right),
$$

and at $x_{s}$, we define

$$
U_{s}=\mathrm{B}\left(x_{s}, \varepsilon_{2}\right), \quad V_{s}=\mathrm{B}\left(x_{s}, \varepsilon_{3}\right) .
$$

By proposition 15, one chooses next for each $y \in Y$ a segment of orbit $Z_{y}$ that connects $V_{s}$ to $V_{y}$ and $\varepsilon_{0}$-visits exactly $X_{s, y}$. This is possible by our choice of the scales $\varepsilon_{1}$ and $\varepsilon_{0}$.

We now define by induction the sets $V_{k} \subset U_{k}$ and the orbit $Z_{k}$ for $k \geq 1$. Let us assume that all the sets $V_{\ell} \subset U_{\ell}$ and the orbits $Z_{\ell}$ for $k \leq \ell \leq s$ have been constructed for some $k \geq 2$. Since the orbits $Z_{\ell}$, with $k \leq \ell \leq s$ and $Z_{y}$, with $y \in Y$ can be chosen disjoint from $X$ (by the second item of proposition 15), one can choose a neighborhood $U_{k-1} \subset \mathrm{B}\left(x_{k-1}, \varepsilon_{2}\right)$ of $x_{k-1}$ disjoint from

$$
Z_{k} \cup \cdots \cup Z_{s-1} \cup \bigcup_{y \in Y} Z_{y} .
$$

The connecting lemma at $x_{k-1}$ associates to the neighborhood $U_{k-1}$ an open neighborhood $V_{k-1} \subset U_{k-1}$. By section 4.1, we choose a segment of orbit $Z_{k-1}$ that connects $V_{k-1}$ to $V_{k}$ and $\varepsilon_{0}$-visits exactly $X_{k-1, k}$.

After $s$ steps, the open neighborhoods $V_{1} \subset U_{1}$ of $x_{1}$ have been defined. One may require in the last step of the previous induction that $U_{1} \subset$ $\mathrm{B}\left(x_{1}, \varepsilon_{3}\right)$.

One now chooses a neighborhood $U_{0} \subset \mathrm{B}\left(x_{0}, \varepsilon_{2}\right)$ of $x_{0}$ disjoint from

$$
Z_{1} \cup \cdots \cup Z_{s-1}
$$

The connecting lemma associates to $U_{0}$ an open neighborhood $V_{0} \subset U_{0} \cap$ $\mathrm{B}\left(x_{0}, \varepsilon_{3}\right)$. One then introduces a segment of orbit $Z_{0}$ that connects $V_{0}$ to $V_{1}$ and $\varepsilon_{2}$-visits exactly the set $X_{0,1}$.

The first two properties of the proposition are clearly satisfied by this construction. The set of points that are visited by these segment of orbits $Z_{k}$ and $Z_{y}$ is also controlled.

By our choice of $U_{k}$, for any $k \in\{1, \ldots, s-1\}$, the open set $U_{k}$ does not intersect the orbits $Z_{y}$ with $y \in Y$ and for any $k \in\{0, \ldots, s-2\}$, the open set $U_{k}$ does not intersect the orbits $Z_{\ell}$ with $\ell \in\{k+1, \ldots, s-1\}$.

For any $k \in\{3, \ldots, s\}$, the open set $U_{k}$ does not intersect the orbits $Z_{\ell}$ with $\ell \in\{1, \ldots, k-2\}$ : indeed, $Z_{\ell} \varepsilon_{0}$-visits exactly the set $X_{\ell, \ell+1}$; by proposition $16 x_{k}$ does not belong to $X_{\ell, \ell+1}$ so that $Z_{\ell}$ does not $\varepsilon_{0}$-visit $x_{k}$; moreover $U_{k}$ is contained in $\mathrm{B}\left(x_{k}, \varepsilon_{0}\right)$. 
Similarly, $U_{k}$ with $k \in\{2, \ldots, s\}$ does not intersect $Z_{0}$ since this orbit $\varepsilon_{2}$-visits exactly $X_{0,1}$ which does not contain $x_{k}$ and since $U_{k} \subset \mathrm{B}\left(x_{k}, \varepsilon_{2}\right)$ by construction.

We end by noting that for any $y \in Y$, the open set $U_{y}$ does not intersect $Z_{\ell}$ with $\ell \in\{1, \ldots, s-1\}$ : this is due to the fact that $Z_{\ell} \varepsilon_{0}$-visits exactly the set $X_{\ell, \ell+1}$ and that by proposition 16, the point $y$ does not belong to $X_{\ell, \ell+1}$. This gives the property since $U_{y}$ is contained in $\mathrm{B}\left(y, \varepsilon_{0}\right)$.

\subsection{Connecting the orbits: the end of the proof of proposition 8}

The end of the proof has now two main steps: we first choose a point $x_{s+1}$ in $Y$ and then apply the connecting lemma at each point $x_{k}$ with $k \in$ $\{1, \ldots, s+1\}$ in order to connect together the orbits $Z_{1}, \ldots, Z_{s}$ and $Z_{s+1}=$ $Z_{x_{s+1}}$. Before the first step, we need however to perform a first perturbation at $x_{1}$ that connects $Z_{0}$ to $Z_{1}$.

In this section, the orbits $Z_{k}$ will be written in the form $\left(z_{k}(0), \ldots, z_{k}\left(n_{k}\right)\right)$ and the orbits $Z_{y}$ in the form $\left(z_{y}(0), \ldots, z_{y}\left(n_{y}\right)\right)$.

4.4.1. Perturbation at $x_{1}$. - The point $p_{1}=z_{0}(0)$ has some positive iterate $z_{0}\left(n_{0}\right)$ in $V_{1}$. The point $q_{1}=z_{1}\left(n_{1}\right)$ has some negative iterate $z_{1}(0)$ in $f^{N}\left(V_{1}\right)$. Hence, one can perturb $f$ by the connecting lemma in the set $U_{1} \cup \cdots \cup f^{N}\left(U_{1}\right)$. This defines a new diffeomorphism $g_{1} \in \mathcal{U}$ and an integer $m_{0} \geq 1$ so that the positive orbit of $z_{0}(0)$ now contains $z_{1}\left(n_{1}\right)=g_{1}^{m_{0}}\left(z_{0}(0)\right)$.

By the connecting lemma, the new orbit $\left(z_{0}(0), \cdots, g_{1}^{m_{0}}\left(z_{0}(0)\right)\right)$ can be cut in three parts:

a. an initial part $\left(z_{0}(0), \cdots, g_{1}^{n_{a}}\left(z_{0}(0)\right)\right)$ which connects $V_{0}$ to $U_{1}$ and has support in

$$
Z_{0} \cup U_{1} \cup \cdots \cup f^{N}\left(U_{1}\right)
$$

b. some central part $\left(g_{1}^{n_{a}}\left(z_{0}(0)\right), \cdots, g_{1}^{n_{a}+N}\left(z_{0}(0)\right)\right)$ with support in

$$
U_{1} \cup \cdots \cup f^{N}\left(U_{1}\right),
$$

c. and the last part $\left(g_{1}^{n_{a}+N}\left(z_{0}(0)\right), \cdots, g_{1}^{m_{0}}\left(z_{0}(0)\right)\right)$ which connects $U_{1}$ to $V_{2}$ and has support in

$$
U_{1} \cup \cdots \cup f^{N}\left(U_{1}\right) \cup Z_{1} .
$$

We will also denote $g_{1}^{n_{a}}\left(z_{0}(0)\right)$ by $p$ and set $m_{1}=m_{0}-n_{a}$.

Lemma 3. - The initial part $\left(z_{0}(0), \cdots, p\right) \varepsilon_{2}$-visits exactly the set $X_{0,1}$. 
Proof. - By proposition 17, $V_{0} \subset \mathrm{B}\left(x_{0}, \varepsilon_{3}\right)$ and $U_{1} \subset \mathrm{B}\left(x_{1}, \varepsilon_{3}\right)$. By definition of the scales $\varepsilon_{2}$ and $\varepsilon_{3}$, by corollary 3, and since $Z_{0}$ was assumed to $\varepsilon_{2}$-visits exactly the set $X_{0,1}$, this is also the case for any sub-orbit of $Z_{0}$ which connects $V_{0}$ to $U_{1}$. In particular, this is the case for the initial part $\left(z_{0}(0), \cdots, g_{1}^{n_{a}}\left(z_{0}(0)\right)\right)$ which contains such a sub-orbit.

Lemma 4. - Every sub-orbit of the last part $\left(g_{1}^{N}(p), \cdots, g_{1}^{m_{1}}(p)\right)$, which connects $U_{1}$ to $U_{2}$, $\varepsilon_{0}$-visits exactly the set $X_{1,2}$.

Proof. - The same argument as in the proof of the previous lemma shows that any sub-orbit of the last part $\left(g_{1}^{n_{a}+N}\left(z_{0}(0)\right), \cdots, g_{1}^{m_{0}}\left(z_{0}(0)\right)\right)$ which connects $U_{1} \subset \mathrm{B}\left(x_{1}, \varepsilon_{1}\right)$ to $U_{2} \subset \mathrm{B}\left(x_{2}, \varepsilon_{1}\right) \varepsilon_{0}$-visits exactly the set $X_{1,2}$. Indeed, such a sub-orbit contains a sub-orbit of $Z_{1}$ which $\varepsilon_{0}$-visits exactly the set $X_{1,2}$.

4.4.2. Choice of $x_{s+1} \in Y$. - In this section, we define the point $x_{s+1} \in Y$. This allows to define $U_{s+1}=U_{x_{s+1}}, V_{s+1}=V_{x_{s+1}}$ and $Z_{s}=Z_{x_{s+1}}$. The other sets $V_{y}, U_{y}$ and orbits $Z_{y}$ with $y \in Y \backslash\left\{x_{s+1}\right\}$ can then be forgotten.

Lemma 5. - There exists a point $x_{s+1} \in Y$ and an integer $n_{q} \in$ $\left\{1, \ldots, n_{a}\right\}$ such that the segment of orbit $\left(g_{1}^{-n_{q}}(p), \ldots, p\right)$ connects $V_{s+1}=$ $V_{x_{s+1}}$ to $U_{1}$. Moreover, this segment of orbit satisfies the following properties:

1. It does not intersect the open sets $U_{k} \cup \cdots \cup f^{N}\left(U_{k}\right)$ for $k \in\{2, \ldots, s\}$.

2. Any sub-orbit of the form $\left(g_{1}^{-\ell}(p), \ldots, p\right), \ell \leq n_{q}$, that connects $U_{s+1}=$ $U_{x_{s+1}}$ to $U_{1} \varepsilon_{0}$-visits $Y \backslash\left\{x_{s+1}\right\}$.

Proof. - Let $n_{b} \in\left\{0, \ldots, n_{a}\right\}$ be the largest integer such that $\left(g_{1}^{n_{b}}\left(z_{0}(0)\right), \ldots, g_{1}^{n_{a}}\left(z_{0}(0)\right)\right)$ intersects every set $f^{N}\left(\mathrm{~B}\left(y, \varepsilon_{0}\right)\right)$ with $y \in Y$. This exists since $\left(z_{0}(0), \ldots, g_{1}^{n_{a}}\left(z_{0}(0)\right)\right)$ $\varepsilon_{2}$-visits $Y \subset X_{0,1}$ by lemma 3 .

Note that since $n_{b}$ is maximal, $g_{1}^{n_{b}}\left(z_{0}(0)\right)$ belongs to some unique set $f^{N}\left(\mathrm{~B}\left(y, \varepsilon_{0}\right)\right)$ with $y \in Y$. One defines the point $x_{s+1}$ by setting $x_{s+1}=y$.

By definition, $n_{b}$ is also the largest integer such that $g_{1}^{n_{b}}\left(z_{0}(0)\right)$ belongs to $f^{N}\left(\mathrm{~B}\left(x_{s+1}, \varepsilon_{0}\right)\right)$ but since the orbit $\left(z_{0}(0), \ldots, p\right) \varepsilon_{2}$-visits $X_{0,1}$, there exists a smaller integer $n_{c} \in\left\{0, \ldots, n_{b}\right\}$ such that $g_{1}^{n_{c}}\left(z_{0}(0)\right)$ belongs to $f^{N}\left(\mathrm{~B}\left(x_{s+1}, \varepsilon_{2}\right)\right)$. (There may be several choices for $n_{c}$.) One sets $n_{q}=n_{a}-n_{c}$.

By construction, the orbit $\left(g_{1}^{-n_{q}}(p), \ldots, p\right)$ connects $V_{s+1}=V_{x_{s+1}}$ to $U_{1}$ since $V_{x_{s+1}}=\mathrm{B}\left(x_{s+1}, \varepsilon_{2}\right)$ by proposition 17 .

By proposition 17, for any $k \in\{2, \ldots, s\}$, the open set $U_{k}$ is contained in $\mathrm{B}\left(x_{k}, \varepsilon_{2}\right)$. Since the set that $\left(g_{1}^{-n_{q}}(p), \ldots, p\right) \varepsilon_{2}$-visits is contained in $X_{0,1}$ 
and since $x_{k}$ does not belong to $X_{0,1}$ by proposition 16, we deduce that $\left(g_{1}^{-n_{q}}(p), \ldots, p\right)$ does not intersect $U_{k} \cup \cdots \cup f^{N}\left(U_{k}\right)$.

Let us consider now a segment of orbit of the form $\left(g_{1}^{-\ell}(p), \ldots, p\right)$ that connects $U_{s+1}=U_{x_{s+1}}$ to $U_{1}$. Since $g^{n_{b}}\left(z_{0}(0)\right)$ is the last point of the sequence $\left(g_{1}^{-n_{a}}(p), \ldots, p\right)$ that belongs to $f^{N}\left(\mathrm{~B}\left(x_{s+1}, \varepsilon_{0}\right)\right)$, one gets that the orbit $\left(g_{1}^{-\ell}(p), \ldots, p\right)$, which intersects $U_{s+1} \subset \mathrm{B}\left(x_{s+1}, \varepsilon_{0}\right)$, contains the orbit $\left(g_{1}^{n_{b}}\left(z_{0}(0)\right), \ldots, g_{1}^{n_{a}}\left(z_{0}(0)\right)\right)$. By our choice of $n_{b}$, one gets that $\left(g_{1}^{-\ell}(p), \ldots, p\right)$ intersects every set $f^{N}\left(\mathrm{~B}\left(y, \varepsilon_{0}\right)\right)$ with $y \in Y$. This shows that this orbit $\varepsilon_{0^{-}}$ visits every point of $Y \backslash\left\{x_{s+1}\right\}$.

4.4.3. Inductive assumptions. - One will now perturb quite independently in the open sets $U_{k}$ for each $2 \leq k \leq s+1$. However one needs to choose carefully the order of these perturbations. Thus, we inductively build (see below) a sequence of perturbed maps $g_{k},(1 \leq k \leq s)$ in $\mathcal{U}$ such that the following properties are satisfied:

1. $g_{k+1}$ is a perturbation of $g_{k}$ with support in

$$
U_{k+1} \cup \cdots \cup f^{N}\left(U_{k+1}\right) \text {. }
$$

2. Some positive iterate $g_{k}^{m_{k}}(p)$ of $p$ by $g_{k}$ hits $z_{k}\left(n_{k}\right)$; the negative iterate $g^{-n_{q}}(p)$ of $p$ belongs to $f^{N}\left(V_{s+1}\right)$.

3. In the segment of orbit $\left(p, \cdots z_{k}\left(n_{k}\right)\right)$ by $g_{k}$, every sub-orbit that connects $U_{1}$ to $U_{k+1} \varepsilon_{0}$-visits exactly the set

$$
\left\{x_{2}, \ldots, x_{k+1}\right\} \cup X_{1,2} \cup \cdots \cup X_{k, k+1} .
$$

Moreover, such an orbit does not intersect the sets $U_{\ell} \cup \cdots \cup f^{N}\left(U_{\ell}\right)$ with $\ell \in\{k+2, \ldots, s+1\}$.

4. In $\left(g_{k}^{-n_{q}}(p), \cdots, p\right)$, every sub-orbit of the form $\left(g_{k}^{-m}(p), \cdots, p\right)$ that connects $U_{s+1}$ to $U_{1} \varepsilon_{0}$-visits all the points in $Y \backslash\left\{x_{s+1}\right\}$. Moreover, it does not intersect the sets $U_{\ell} \cup \cdots \cup f^{N}\left(U_{\ell}\right)$ with $\ell \in\{2, \ldots, s\}$.

4.4.4. Definition of the perturbations $g_{k+1}$. - In order to define $g_{k+1}$ from $g_{k}$, let us consider again the point $p$. Recall that it does not belongs to $U_{k+1} \cup \cdots \cup f^{N}\left(U_{k+1}\right)$ and has some iterate $g_{k}^{m_{k}}(p)$ in $V_{k+1}$.

Since the segment of orbit orbit $Z_{k+1}$ under $f$ does not intersect the support of the perturbation $g_{k}$ of $f$, it is also an orbit of $g_{k}$ that goes from $z_{k+1}(0) \in f^{N}\left(V_{k+1}\right)$ to $q_{k+1}=z_{k+1}\left(n_{k+1}\right) \in V_{k+2}$.

One can apply the connecting lemma at $x_{k+1}$ on the map $g_{k}$ between the points $p$ and $q_{k+1}$. This defines a new diffeomorphism $g_{k+1}$. Since the support of this new perturbation is disjoint from the previous one, $g_{k+1}$ is contained in $\mathcal{U}$ (recall section 2.1). 
Some positive iterate of $p$ under $g_{k+1}$ hits now $q_{k+1}$. Since the former orbit $p, \cdots, z_{k}\left(n_{k}\right)$ (resp. $\left.z_{k+1}(0), \cdots, z_{k+1}\left(n_{k+1}\right)\right)$ did not intersect the set $U_{k+2}$ (resp. the set $\left.U_{1}\right)$, any sub-orbit $Z^{\prime}$ of $\left(p, \cdots, q_{k+1}\right)$ By $g_{k+1}$ that connects $U_{1}$ to $U_{k+2}$ intersects $U_{k+1}$ by the connecting lemma. Thus, $Z^{\prime}$ contains some sub-orbits of the orbits $p, \cdots, z_{k}\left(n_{k}\right)$ and $\left(z_{k+1}(0), \cdots, q_{k+1}\right)$ by $g_{k}$ which connect $U_{1}$ to $U_{k+1}$ and $U_{k+1}$ to $U_{k+2}$ respectively. This implies by item (3) of section 4.4 .3 for $g_{k}$ and by corollary 3 that $Z^{\prime} \varepsilon_{0}$-visits exactly the set

$$
\left\{x_{2}, \cdots, x_{k+2}\right\} \cup X_{1,2} \cup \cdots \cup X_{k+1, k+2} .
$$

The orbit under $g_{k}$ from $g_{k}^{-n_{q}}(p)$ to $p$ remains unchanged since it does not intersect the support of the new perturbation. Hence, property (4) of section 4.4 .3 still holds. This ends the construction of the perturbed maps $g_{k}, 0 \leq k \leq s$.

4.4.5. The last perturbation $g_{s+1}$. - One builds finally a perturbation $g_{s+1}$ of $g_{s}$ with support in $U_{s+1} \cup \cdots \cup f^{N}\left(U_{s+1}\right)$ in order to close the orbit $\left(g_{s}^{-n_{q}}(p), \cdots, p, \cdots, z_{s}\left(n_{s}\right)=g_{s}^{m_{s}}(p)\right)$.

Let us define $n_{p}=m_{s}$ in order to apply theorem 5. Recall that $p$ does not belongs to $U_{s+1} \cup \cdots \cup f^{N}\left(U_{s+1}\right)$ and has some forward iterate $g_{s}^{n_{p}}(p)$ in $V_{s+1}$. The point $q=p$ has also some backward iterate $g_{s}^{-n_{q}}(q)$ in $f^{N}\left(V_{s+1}\right)$.

One more time, one applies the connecting lemma (theorem 5), at $x_{s+1}$ on the map $g_{s}$ between the points $p$ and $q$. This defines a new diffeomorphism $g_{k+1}$. Since the support of this new perturbation is disjoint from the previous one, $g_{s+1}$ is contained in $\mathcal{U}$ (recall Section 2.1).

4.4.6. Conclusion of the proof. - The map $g_{s+1}$ sends by positive iterations $p$ on $q=p$ so that $p$ now is periodic. This orbit (by $g_{s+1}$ ) contains some sub-orbit of $\left(p, \cdots, g_{s}^{m_{s}}(p)\right)$ by $g_{s}$ connecting $U_{1}$ to $U_{s+1}$. Hence, by property (3) of section 4.4.3 the orbit of $p \varepsilon_{0}$-visits the set

$$
\left\{x_{2}, \cdots, x_{s+1}\right\} \cup X_{1,2} \cup \cdots \cup X_{s, s+1} .
$$

Moreover it contains also a sub-orbit $\left(g_{s}^{-n}(p), \cdots, p\right)$ of $\left(g_{s}^{-n_{q}}(p), \cdots, p\right)$ that connects $U_{s+1}$ to $U_{1}$. By property (4) of section 4.4.3, it must $\varepsilon_{0}$-visits all the points in $Y \backslash\left\{x_{s+1}\right\}$. Note also that $p$ belongs to $B\left(x_{1}, \varepsilon_{0}\right)$. This shows that the orbit of $p \varepsilon_{0}$-visits also $x_{1}$.

By proposition 16 (1) and the definition of $Y$, the periodic orbit we have built $\varepsilon_{0}$-visit every point in $X$ : it intersects every ball $\mathrm{B}(x, \eta)$ with $x \in X$. On the other hand, the periodic orbit is made of segment of orbits $Z_{k}$ and $Z_{y}$ and of points contained in the support of the perturbations. By construction, the periodic orbit is thus contained in the open set $W$ introduced at the beginning of section 4 . This ends the proof of the proposition 8 . 


\section{Proof of the other perturbation results}

\subsection{Approximation by finite segments of orbits (proof of proposition 9)}

As in section 1 , one considers a diffeomorphism $f$ and a neighborhood $\mathcal{U}$ of $f$ in $\operatorname{Diff}^{1}(M)$ which satisfies condition $(\mathrm{F})$. An integer $N \geq 1$ is given by the connecting lemma. Let $W \subset M$ be an open set containing a finite set $X=\left\{p_{1}, \ldots, p_{r}\right\}$ that satisfies the assumptions of proposition 9. We also choose a constant $\eta>0$.

The proof of the proposition is very similar but simpler than the proof of proposition 8. One difficulty in the proof of proposition 8 was to close the orbit. This justified the introduction of the set $Y$ and the delicate choice of the point $x_{s+1}$ (see sections 4.2 and 4.4.2). In this section, only two scales $\varepsilon_{1}<\varepsilon_{0}$ in $(0, \eta)$ will be used (see section 4.0.

As in section 4.1, one considers finite segments of orbits contained in $W$. One defines a set $X_{x, x^{\prime}} \subset X$ for each pair $\left(x, x^{\prime}\right)$ of points in $X$ such that $x \prec_{W} x^{\prime}$. Then, one adapts proposition 16:

Proposition 18. - There exists a sequence $\left(x_{0}, \cdots, x_{s}\right)(s \geq 1)$ of distinct points of $X$ with the following properties:

1. $x_{0}=p_{1}$.

2. $x_{k} \prec_{W} x_{k+1}$ for any $1 \leq k \leq s-1$;

3. $X=\left\{x_{0}, \cdots, x_{s}\right\} \cup X_{0,1} \cup \cdots \cup X_{s-1, s}$;

4. for any $2 \leq k \leq s$, the point $x_{k}$ does not belong to

$$
\left\{x_{0}, x_{1}, \ldots, x_{k-1}\right\} \cup X_{0,1} \cup X_{1,2} \cup \cdots \cup X_{k-2, k-1} \text {. }
$$

Proof. - By assumption, the set $X$ is ordered by $\prec_{W}$. One sets $x_{0}=p_{1}$. Our hypothesis implies that for any $x \in X \backslash\left\{x_{0}\right\}$ we have $x_{0} \prec_{W} x$.

One builds the sequence $\left(x_{k}\right)$ inductively and assumes at each step that

$$
x_{k} \prec_{W} x \text { for any } x \notin\left\{x_{0}, \cdots, x_{k}\right\} \cup X_{0,1} \cup \cdots \cup X_{k-1, k} .
$$

Each time the point $x_{k}$ has been defined, one can order

$$
X \backslash\left\{x_{1}, \cdots, x_{k}\right\} \cup X_{1,0} \cup \cdots \cup X_{k-1, k}
$$

as a sequence $\left(\tilde{p}_{1}, \tilde{p}_{2}, \cdots, \tilde{p}_{m}\right)$ such that $\tilde{p}_{i} \prec_{W} \tilde{p}_{j}$ for each $0 \leq i<j \leq m$ (there may be several possibilities) and one chooses $x_{k+1}=\tilde{p}_{1}$. Then the inductive assumption (5) is clearly satisfied.

In order to conclude the proof of proposition 9, one chooses inductively (as $k$ decreases): 
1. some neighborhoods $V_{k} \subset U_{k} \subset \mathrm{B}\left(x_{k}, \varepsilon_{1}\right)$ of $x_{k}$ that satisfy the connecting lemma at $x_{k}$,

2. a segment of orbit $Z_{k}=\left(z_{k}(0), \cdots, z_{k}\left(n_{k}\right)\right)$ connecting $V_{k}$ to $V_{k+1}$ that $\varepsilon_{0}$-visits exactly the set $X_{k, k+1}$ and that is contained in $W$.

One may assume that $Z_{k}$ is disjoint from any $U_{\ell}$ with $\ell \neq k, k+1$. Then, one sets $p=p_{1}=x_{0}$ so that $p$ belongs to $V_{0}$.

Since $z_{0}(0)$ belongs to $f^{N}\left(V_{0}\right)$, the connecting lemma between the points $f^{-1}(p)$ and $f\left(z_{0}(0)\right)$ builds a perturbation $g_{0} \in \mathcal{U}$ of $f$ with support in $U_{0}, \ldots, f^{N-1}\left(U_{0}\right)$ and such that $f^{N}(p)=z_{0}(0)$. Hence, the segments of orbits $Z_{k}$ with $k \in\{1, \ldots, s-1\}$ do not intersect the support of this perturbation. One now builds a sequence of perturbations $\left(g_{k}\right)_{1 \leq k \leq s-1}$ in $\mathcal{U}$ such that

1. $g_{k+1}$ belongs to $\mathcal{U}$ and is a perturbation of $g_{k}$ with support in

$$
U_{k+1} \cup \cdots \cup f^{N-1}\left(U_{k+1}\right) ;
$$

2. $\quad g_{k}$ sends $p$ on $z_{k}\left(n_{k}\right)=g_{k}^{m_{k}}(p)$;

3. in the orbit $\left(p, \cdots, g_{k}^{m_{k}}(p)\right)$ of $g_{k}$, every sub-orbit of the form $\left(p, \cdots, g_{k}^{m}(p)\right)$ that intersects $U_{k+1} \quad \varepsilon_{0}$-visits exactly the set

$$
\left\{x_{0}, \cdots, x_{k+1}\right\} \cup X_{0,1} \cup \cdots \cup X_{k, k+1} .
$$

The orbit $\left(p, \cdots, g_{s-1}^{m_{k-1}}(p)\right)$, for the last perturbation $g=g_{s-1}, \varepsilon_{0}$-visits all the points of $X$ and is contained in $W$ as required.

The support of the perturbation $g$ is contained in the balls $f^{j}\left(\mathrm{~B}\left(x_{k}, \eta\right)\right)$ with $j \in\{0, \ldots, N-1\}$ and $k \in\{0, \ldots, s-1\}$.

Remark. - From the conclusion of the proof, one sees that one gets a stronger statement for proposition 9: let $x \in\left\{p_{1}, \ldots, p_{r}\right\}$ be the last point visited by the orbit $\left(p_{1}, \ldots, g^{n}\left(p_{1}\right)\right)$ (i.e. choosing $n$ minimal, one may have $\left.g^{n}\left(p_{1}\right) \in \mathrm{B}(x, \eta)\right)$. Then, the support of the perturbation $g$ of $f$ is only contained in the balls $f^{j}\left(\mathrm{~B}\left(p_{k}, \eta\right)\right)$ with $j \in\{0, \ldots, N-1\}$ and $k \in\{1, \ldots, r\}$ such that $p_{k} \neq x$.

5.2. Asymptotic approximation (proof of proposition 10)

Let $f$ be a diffeomorphism that satisfies condition $(\mathrm{A})$ and $\mathcal{U}$ a neighborhood of $f$ in $\operatorname{Diff}^{1}(M)$ that satisfies condition $(\mathrm{F})$. In order to control an infinite half orbit, one will perform an infinite sequence of perturbation:

- from the relation $x \prec_{\hat{W}_{A}} A$, one introduces a sequence of intermediary points $\left(y_{n}\right)$ such that

$$
x \prec_{\hat{W}_{A}} y_{1} \prec_{W_{A}} y_{2} \prec_{W_{A}} \cdots \prec_{W_{A}} A ;
$$


- one then perturbs at each point $y_{n}$ in order to connect all these points together.

For the second step of the proof, one should check the convergence of the sequence of perturbation: one considers a decreasing sequence of neighborhoods $\left(\mathcal{V}_{k}\right)$ of Id in $\operatorname{Diff}^{1}(M)$ which satisfy the property $(\mathrm{F})$.

$(F)$ For any perturbations $\varphi$ and $\varphi^{\prime}$ of Id in $\mathcal{V}_{k}$ with disjoint support, the composed perturbation $\varphi \circ \varphi^{\prime}$ belongs to $\mathcal{V}_{k}$.

Hence the set $\mathcal{U}_{k}$ of diffeomorphisms $g$ of the form $\varphi \circ f$ with $\varphi \in \mathcal{V}_{k}$ is a neighborhood of $f$ which satisfies the property $(\mathrm{F})$. One will assume that $\cap_{k} \mathcal{V}_{k}=\{\operatorname{Id}\}$ and $\mathrm{Cl}\left(\mathcal{U}_{0}\right) \subset \mathcal{U}$.

The connecting lemma associates to each pair $\left(f, \mathcal{U}_{k}\right)$ an integer $N_{k} \geq 1$.

5.2.1. Intermediary points. - We consider a closed invariant set $A$.

The following lemma allows to introduce intermediary points for the stable set:

Lemma 6. - For any neighborhoods $W_{i} \subset W_{e}$ of $A$ and any point $y \in W_{e}$ such that $y \prec_{W_{e}} A$, there exists $y^{\prime} \in W_{i} \backslash A$ such that $y \prec_{W_{e}} y^{\prime}$ and $y^{\prime} \prec_{W_{i}}$ A. Moreover, the forward orbit of $y^{\prime}$ is contained in $W_{i}$.

Proof. - Let us take a smaller neighborhood $V$ of $A$ such that $\mathrm{Cl}(V) \subset$ $W_{i}$. One considers for any $k \geq 1$, a finite segment of orbit $\left(z_{0}^{k}, \ldots, z_{n(k)}^{k}\right)$ in $W_{e}$ such that $z_{0}^{k}$ belongs to the ball $\mathrm{B}(y, 1 / k)$ and $z_{n(k)}^{k}$ to the $1 / k$ neighborhood of $A$. For $k$ large enough, $z_{n(k)}^{k}$ belongs also to the set $V$. One then considers the first point $z_{m(k)}^{k}$ of the orbit $\left(z_{0}^{k}, \ldots, z_{n(k)}^{k}\right)$ such that $\left(z_{m(k)}^{k}, \ldots, z_{n(k)}^{k}\right)$ is contained in $V$.

One can extract a subsequence of $\left(z_{m(k)}\right)_{k}$ and assume that it converges to a point $y^{\prime} \in \mathrm{Cl}(V) \backslash A$. The sequences $\left(z_{0}^{k}, \ldots, z_{m(k)}^{k}\right)$ are contained in $W_{e}$ and show that $y \prec_{W_{e}} y^{\prime}$. Similarly, the sequences $\left(z_{m(k)}^{k}, \ldots, z_{n(k)}^{k}\right)$ are contained in $W_{i}$ and show that $y^{\prime} \prec_{W_{i}} A$, as required. Since the sequences $\left(z_{m(k)}^{k}, \ldots, z_{n(k)}^{k}\right)$ are contained in $\mathrm{Cl}(V)$, since $\left(z_{m(k)}^{k}\right)$ converges to $y^{\prime}$ and since $z_{n(k)}^{k}$ becomes arbitrarily close to the invariant set $A$, the difference $n(k)-m(k)$ goes to infinity and the forward orbit of $y^{\prime}$ is contained in $\mathrm{Cl}(V) \subset W_{i}$.

We now introduce as in the statement of proposition 10 two open neighborhoods $W_{A} \subset \hat{W}_{A}$ of $A$, with $\mathrm{Cl}\left(W_{A}\right) \subset \hat{W}_{A}$, and a point $x \in \hat{W}_{A}$ such that $x \prec_{\hat{W}_{A}} A$. We choose a neighborhood $U$ of $x$. One may assume that $U \subset \hat{W}_{A} \backslash A$. 
We build the open sets where the perturbations will be performed:

Proposition 19. - There exist three sequences $\left(U_{k}\right)_{k \geq 1},\left(V_{k}\right)_{k \geq 1}$ and $\left(W_{k}\right)_{k \geq 0}$ of open sets and a sequence of finite segment of orbits $\left(z_{0}^{k}, z_{1}^{k}, \ldots, z_{n(k)}^{k}\right)_{k \geq 0}$ such that:

1. $\left(W_{k}\right)$ is a decreasing sequence of neighborhoods of $A$ such that $W_{0} \subset$ $\hat{W}_{A}, W_{1} \subset W_{A}$ and $\cap_{k} W_{k}=A$.

2. The orbit $\left(z_{0}^{k}, z_{1}^{k}, \ldots, z_{n(k)}^{k}\right)$ is contained in $W_{k} \backslash W_{k+2}$.

3. The connecting lemma may be applied to the open sets $V_{k} \subset U_{k}$ with the time $N_{k}$ for $f$ and the neighborhood $\mathcal{U}_{k}$; moreover, the iterates $U_{k}, f\left(U_{k}\right), \ldots, f^{N_{k}}\left(U_{k}\right)$ are all contained in $W_{k}$ and have their closure disjoint from $W_{k+1}$.

4. The points $z_{n(k)}^{k}$ and $z_{0}^{k+1}$ are contained in $V_{k+1}$; the point $z_{0}^{0}$ belongs to $U$.

Proof. - In the proof, it will be useful to introduce also the open sets $V_{0}=U_{0}=U$ (however the connecting lemma will not be used for $\left(V_{0}, U_{0}\right)$ ) and to builds a sequence $\left(y_{k}\right)_{k \geq 0}$ such that

- $y_{k} \in V_{k}$, moreover $y_{k} \prec_{W_{k}} y_{k+1}$ and $y_{k} \prec_{W_{k}} A$;

- when $k \geq 1$, the point $y_{k}$ is not a periodic point of period less than or equal to $N_{k}$;

- when $k \geq 1$, the forward orbit of $y_{k}$ is contained in $W_{k}$.

We now start the construction. The first open set $W_{0}$ is chosen equal to $\hat{W}_{A}$. One sets $y_{0}=x$.

The constructions are then done by induction on $k$ : one assumes that $y_{k}, U_{k}, V_{k}, W_{k}$ and (if $\left.k \geq 1\right)\left(z_{0}^{k-1}, z_{1}^{k-1}, \ldots, z_{n(k-1)}^{k-1}\right)$ have been defined and one builds $U_{k+1}, V_{k+1}, W_{k+1}$ and the sequence $\left(z_{0}^{k}, z_{1}^{k}, \ldots, z_{n(k)}^{k}\right)$.

One first chooses a neighborhood $W_{k+1}$ of $A$ whose closure is contained in $W_{k}$ and disjoint from the closures of the iterates $U_{k}, \ldots, f^{N_{k}}\left(U_{k}\right)$ and (if $k \geq 1$ ) from $\left(z_{0}^{k-1}, z_{1}^{k-1}, \ldots, z_{n(k-1)}^{k-1}\right)$. Recall that by assumption (A) on $f$, the periodic points of period less than or equal to $N_{k+1}$ are finite and hence isolated. One thus can choose $W_{k+1}$ such that $W_{k+1} \backslash A$ does not contain any periodic point of period less than or equal to $N_{k+1}$. One also chooses $W_{k+1}$ in a $1 /(k+1)$-neighborhood of $A$. For $k=0$, one can assume that $W_{1}$ is contained in $W_{A}$.

One can then apply lemma 6 to the point $y=y_{k}$ and to the open sets $W_{e}=W_{k}$ and $W_{i}=W_{k+1}$. This defines a new point $y_{k+1}$ whose forward orbit is contained in $W_{i}$. Moreover $y_{k} \prec_{W_{k}} y_{k+1}$ and $y_{k+1} \prec_{W_{k+1}} A$. As $y_{k+1}$ belongs to $W_{k+1} \backslash A$, it is not a periodic point of period less than or equal to $N_{k+1}$. 
Since the forward orbit of $y_{k+1}$ is contained in $W_{k+1}$ and since $y_{k+1}$ is not a periodic point of period less than or equal to $N_{k+1}$, one can build two neighborhoods $V_{k+1} \subset U_{k+1}$ of $y_{k+1}$ where the connecting lemma can be applied with the time $N_{k+1}$ to $f$ and the neighborhood $\mathcal{U}_{k+1}$. Choosing $U_{k+1}$ small enough, one can assume that the closure of the iterates $U_{k+1}, \ldots, f^{N_{k+1}}\left(U_{k+1}\right)$ are all contained in $W_{k+1}$ and are disjoint from $A$.

By the relation $y_{k} \prec_{W_{k}} y_{k+1}$, there exists a finite segment of orbit $\left(z_{0}^{k}, z_{1}^{k}, \ldots, z_{n(k)}^{k}\right)$ contained in $W_{k}$ such that $z_{0}^{k}$ belongs to $V_{k}$ and $z_{n(k)}^{k}$ belongs to $V_{k+1}$. By this induction, all the required properties are satisfied. We get $A=\cap_{k} W_{k}$ since $W_{k}$ is contained in a $1 / k$-neighborhood of $A$.

5.2.2. Infinite sequence of perturbations. - One sets $z=z_{0}^{0}$. We then define a sequence of intermediate perturbations.

Proposition 20. - There is a sequence of perturbations $\left(g_{k}\right)$ of $f$ and an increasing sequence of integers $\left(n_{k}\right)$ larger than 1 such that:

1. $g_{0}=f$ and $n_{0}=0$.

2. For $k \geq 1, g_{k}=\varphi_{k} \circ g_{k-1}$ and $\varphi_{k}$ is a perturbation of Id with support in $U_{k} \cup \cdots \cup f^{N_{k}-1}\left(U_{k}\right)$. Moreover $\varphi_{k}$ belongs to $\mathcal{V}_{k}$.

3. For $\ell \in\{0, \ldots, k-1\}$, the orbit $\left(g_{k}^{n_{\ell}}(z), g_{k}^{n_{\ell}+1}(z), \ldots, g_{k}^{n_{\ell+1}}(z)\right)$ by $g_{k}$ is contained in $W_{\ell} \backslash W_{\ell+2}$.

Proof. - The sequence is built inductively and will satisfy the following additional property:

- There exists an integer $m_{k}>n_{k}$ such that $g_{k}^{m_{k}}(z)$ belongs to $V_{k+1}$.

- The orbit $\left(g_{k}^{n_{k}}(z), \ldots, g_{k}^{m_{k}}(z)\right)$ is contained in $W_{k} \backslash W_{k+2}$.

We define $g_{0}=f$ and $n_{0}=0$. By $g_{0}$, the point $z$ has a forward iterate $f^{n(0)}(z)=z_{n(0)}^{0}$ in $V_{1}$. One sets $m_{0}=n(0)$. The inductive assumptions are satisfied for $k=0$.

We now assume that the perturbation $g_{k}$, the integers $\left(n_{\ell}\right)_{\ell \leq k}$ and the integer $m_{k}$ have been defined. We explain how to define the perturbation $g_{k+1}$ and the integers $n_{k+1}$ and $m_{k+1}$.

The forward orbit of $g_{k}^{n_{k}}(z)$ has an iterate $g_{k}^{m_{k}}(z)$ in $V_{k+1}$ and the backward orbit of $z_{n(k+1)}^{k+1}$ has an iterate $z_{0}^{k+1}$ in $V_{k+1}$. Moreover these segments of orbits are contained in $W_{k} \backslash W_{k+3}$. One can apply the connecting lemma at the sets $V_{k+1} \subset U_{k+1}$ to $\left(g_{k}, \mathcal{U}_{k+1}\right)$ since $g_{k}$ and $f$ coincide on $U_{k+1} \ldots, f^{N_{k+1}}\left(U_{k+1}\right)$. The new perturbation is of the form $g_{k+1}=\varphi_{k+1} \circ g_{k}$ and $\varphi_{k+1} \circ f$ belongs to $\mathcal{U}_{k+1}$. Hence, $\varphi_{k+1} \in \mathcal{V}_{k+1}$.

By the new diffeomorphism $g_{k+1}$, the point $g_{k}^{n_{k}}(z)$ has a forward iterate $g_{k+1}^{m_{k+1}}(z)$ equal to $z_{n(k+1)}^{k+1}$, in $V_{k+2}$. There also exists an iterate $g_{k+1}^{n_{k+1}}(z)$ with $n_{k}<n_{k+1}<m_{k+1}$ such that: 
- The segment of orbit $\left(g_{k+1}^{n_{k}}(z), \ldots, g_{k+1}^{n_{k+1}}\right)$ is contained in the union of $\left\{g_{k}^{n_{k}}(z), \ldots, g_{k}^{m_{k}}\right\}$ with the open sets $V_{k+1}, f\left(V_{k+1}\right), \ldots, f^{N_{k+1}}\left(V_{k+1}\right)$. Hence, it is contained in $W_{k} \backslash W_{k+2}$.

- The segment of orbit $\left(g_{k+1}^{n_{k+1}}(z), \ldots, g_{k+1}^{m_{k+1}}\right)$ is contained in the union of $\left\{z_{0}^{k+1}, \ldots, z_{n(k+1)}^{k+1}\right\}$ with the open sets $V_{k+1}, f\left(V_{k+1}\right), \ldots, f^{N_{k+1}}\left(V_{k+1}\right)$. Hence, it is contained in $W_{k+1} \backslash W_{k+3}$.

By construction, the orbits $\left(z, g_{k}(z), \ldots, g_{k}^{n_{k}}(z)\right)$ and $\left(z, g_{k+1}(z), \ldots, g_{k+1}^{n_{k}}(z)\right)$ coincide. Hence, the inductive assumption are verified for $k+1$.

We can now conclude the proof of the proposition:

Proof (End of the proof of proposition 119). - We are now ready to introduce the perturbation $g$. Since for $i<j$, the supports $U_{i} \cup \cdots \cup f^{N_{i}-1}\left(U_{i}\right)$ and $U_{j} \cup \cdots \cup f^{N_{j}-1}\left(U_{j}\right)$ of the perturbations $\varphi_{i}$ and $\varphi_{j}$ are pairwise disjoint, since $\mathcal{V}_{j} \subset \mathcal{V}_{i}$ and since $\mathcal{U}_{i}$ satisfies property $(\mathrm{F})$, the perturbations $\varphi_{\ell} \circ \varphi_{\ell+1} \circ$ $\cdots \circ \varphi_{k}$ with $k \leq \ell$ belong to $\mathcal{V}_{k}$. This shows that the sequence $\left(\varphi_{0} \circ \cdots \circ \varphi_{k}\right)_{k}$ converges in the $C^{1}$-topology towards a diffeomorphism $\varphi \in \mathrm{Cl}\left(\mathcal{V}_{0}\right)$. In other words, the sequence $\left(g_{k}\right)$ converges towards a diffeomorphism $g \in \mathrm{Cl}\left(\mathcal{U}_{0}\right) \subset \mathcal{U}$.

The diffeomorphism $g$ coincides with $\varphi_{k} \circ f$ in $U_{k} \cup \cdots \cup f^{N-1}\left(U_{k}\right)$ and with $f$ elsewhere. Consequently, $g$ is a perturbation of $f$ with support contained in $W_{1} \subset W_{A}$ and which coincides with $f$ on $A$. From property (3) of proposition 20, the orbit $\left(g^{n}(z)\right)_{n \geq n_{k}}$ of $z$ by $g$ is contained in $W_{k}$. One deduces that the forward orbit of $z$ by $g$ is contained in $\hat{W}_{A}$ and that its $\omega$-limit set is contained in $A$. This ends the proof of proposition 10 .

\subsection{Approximation by full orbit (proof of theorem [)}

Let $f$ be a diffeomorphism that satisfies condition (A) (the periodic points are "isolated"), $\mathcal{U}$ a neighborhood of $f$ in $\operatorname{Diff}^{1}(M)$ that satisfies condition (F) (allowing the composition of perturbations with disjoint supports), $\mathcal{X}$ a weak orbit and $\eta_{0}>0$ a small constant.

If $\mathcal{X}$ is weakly transitive, there exists a perturbation $g \in \mathcal{U}$ and a periodic orbit $\mathcal{O}$ of $g$ which is at distance less than $\eta_{0}$ from $\mathcal{X}$ in the Hausdorff topology, by theorem 3. This proves theorem 2 in this case. Hence, we will assume that $\mathcal{X}$ is not weakly transitive.

It is simpler to create and to control a forward (or a backward) orbit rather than a full orbit. Hence, the idea of the proof will be to first decompose a subset of $\mathcal{X}$ as a union $\mathcal{X}_{\alpha} \cup \mathcal{O}\left(z_{0}\right) \cup \mathcal{X}_{\omega}$ by using proposition 7 and then to create indenpendently a forward orbit that is close to $\mathcal{X}_{\omega}$ and a backward orbit that is close to $\mathcal{X}_{\alpha}$ for the Hausdorff topology. 
5.3.1. Reduction to half orbits. - The following proposition is an analogue of theorem 2 for half orbits and "generalizes" proposition 10 .

Proposition 21. - Let $f$ be a diffeomorphism that satisfies condition (A) and $\mathcal{U}$ a neighborhood of $f$ in $\operatorname{Diff}^{1}(M)$. We consider:

- a weak orbit $\mathcal{X}_{\omega}$ whose relation $\prec_{\mathcal{X}_{\omega}}$ is transitive,

- a neighborhood $\hat{W}_{\omega}$ of $\mathcal{X}_{\omega}$,

- a point $z_{0} \in \hat{W}_{\omega} \backslash \mathcal{X}$ such that $z_{0} \prec_{\hat{W}_{\omega}} \mathcal{X}_{\omega}$.

We assume moreover that:

(*.1) The points $z_{0}$ and $\mathcal{X}_{\omega}$ are contained in a weak orbit $\mathcal{X}$.

(*.2) We have $z_{0} \prec_{\hat{K}_{\omega}} \mathcal{X}_{\omega}$ where $\hat{K}_{\omega}$ is a compact set contained in $\mathcal{X} \cap$ $\hat{W}_{\omega}$.

(*.3) For any $y \in \mathcal{X}_{\omega}$ and $z \in \mathcal{X} \backslash \mathcal{X}_{\omega}$, the relation $y \prec \mathcal{X} z$ is not satisfied.

Then, for any $\eta_{0}>0$, for any neighborhoods $U_{z_{0}}$ of $z_{0}$ and $W_{\omega}$ of $\mathcal{X}_{\omega}$, there exist a perturbation $g_{\omega} \in \mathcal{U}$ of $f$ with support in $W_{\omega}$, a point $z_{\omega} \in U_{z_{0}}$ and an iterate $g_{\omega}^{n_{\omega}}\left(z_{\omega}\right)$ such that

- the forward orbit of $z_{\omega}$ by $g_{\omega}$ is contained in $\hat{W}_{\omega}$,

- the closure of the forward orbit of $g_{\omega}^{n_{\omega}}\left(z_{\omega}\right)$ is $\eta_{0}$-close to $\mathcal{X}_{\omega}$ for the Hausdorff topology.

Of course, one gets a similar statement for backwards half orbits if one applies the previous proposition to $f^{-1}$.

The proof of the proposition is postponed to the next section. The idea is to approximate the set $\mathcal{X}_{\omega}$ by a finite segment of orbit $Z_{b}$ (by using proposition 9). By the connecting lemma, one can connect a point $z_{\omega}$, close to $z_{0}$, to the first point of $Z_{b}$, by a finite segment of orbit $Z_{a}$. By a last perturbation (given by proposition 10), one can control the forward orbit of the last point in $Z_{b}$ : it stays in a small neighborhood of $\mathcal{X}_{\omega}$ and accumulates on a subset.

The assumption $\left(^{*}\right)$ is technical: it simplifies the proof but it could maybe be removed.

Proof (End of the proof of theorem [2). - The connecting lemma applied to $(f, \mathcal{U})$ gives an integer $N$. We denote by $W$ the $\eta_{0}$-neighborhood of $\mathcal{X}$. We only have to consider the case where $\mathcal{X}$ is not weakly transitive: using proposition 0, there exist some weak orbits $\mathcal{X}_{\alpha}, \mathcal{X}_{\omega}$ (whose relations $\prec \mathcal{X}_{\alpha}$ and $\prec_{\mathcal{X}_{\omega}}$ are transitive) and a point $z_{0}$ such that:

1. The sets $\mathcal{X}_{\alpha}, \mathcal{X}_{\omega}$ and the orbit $\mathcal{O}\left(z_{0}\right)$ of $z_{0}$ are contained in $\mathcal{X}$ and are pairwise disjoint. 
2. The set $\operatorname{Cl}\left(\mathcal{X}_{\alpha} \cup \mathcal{O}\left(z_{0}\right) \cup \mathcal{X}_{\omega}\right)$ is $\eta_{0} / 2$-close to $\mathcal{X}$ in the Hausdorff topology.

3. For any $x \in \mathcal{X}_{\alpha}$ and $z \in \mathcal{X} \backslash \mathcal{X}_{\alpha}$ we have $x \prec \mathcal{X} z$ but the relation $z \prec \mathcal{X} x$ is not satisfied.

4. For any $y \in \mathcal{X}_{\omega}$ and $z \in \mathcal{X} \backslash \mathcal{X}_{\omega}$ we have $z \prec \mathcal{X}$ y but the relation $y \prec \mathcal{X} z$ is not satisfied.

5. The point $z_{0}$ is non-periodic (by remark 1.6.3 after proposition 7).

Let $\hat{K}_{\omega}$ be the compact set obtained by removing from $K$ a small open neighborhood of $\mathcal{X}_{\alpha}$. By item 3), for any points $x \in \mathcal{X} \backslash \hat{K}_{\omega}$, the relation $z_{0} \prec \mathcal{X} x$ does not hold. One deduces that if we have $z_{0} \prec \mathcal{X} y$ for some point $y$, then $z_{0} \prec_{\hat{K}_{\omega}} y$. By item 4 ), this is satisfied for any point $y \in \mathcal{X}_{\omega}$. For any neighborhood $\hat{W}_{\omega}$ of $\hat{K}_{\omega}$ (to be defined later), the assumptions of proposition 21 are satisfied for $f$ by the sets $\mathcal{X}_{\omega}, \hat{W}_{\omega}, \mathcal{X}$ and $\hat{K}_{\omega}$.

One defines similarly a compact set $\hat{K}_{\alpha}$ by removing from $K$ a small neighborhood of $\mathcal{X}_{\omega}$, so that the assumptions of proposition 21 are satisfied for $f^{-1}$ by the sets $\mathcal{X}_{\alpha}, \hat{W}_{\alpha}, \mathcal{X}$ and $\hat{K}_{\alpha}$ where $\hat{K}_{\alpha}$ is any neighborhood of $\hat{K}_{\alpha}$. By choosing some small neighborhoods $W_{\alpha}, U_{z_{0}}, W_{\omega}$ of $\mathcal{X}_{\alpha}, z_{0}$ and $\mathcal{X}_{\omega}$, one gets the following properties:

- For any perturbation $g$ of $f$ with support in $W_{\alpha} \cup W_{\omega} \cup \bigcup_{-N \leq k \leq N} f^{k}\left(U_{z_{0}}\right)$, the $\eta_{0} / 2$-neighborhood of the orbit of any point $z \in U_{z_{0}}$ by $g$ contains the orbit of $z_{0}$ by $f$ (by item 1 )).

- The $N$ first backward and forward iterates of $U_{z_{0}}$ are pairwise disjoint and disjoint from $W_{\alpha}$ and $W_{\omega}$ (by items 1) and 5)).

The neighborhood $\hat{W}_{\omega}$ of $\hat{K}_{\omega}$ is taken equal to $W \backslash \mathrm{Cl}\left(W_{\alpha}\right)$ and the neighborhood $\hat{W}_{\alpha}$ of $\hat{K}_{\alpha}$ is taken equal to $W \backslash \mathrm{Cl}\left(W_{\omega}\right)$

Applying proposition 21 to $f$ and $f^{-1}$, one obtains two perturbation $g_{\omega}$ and $g_{\alpha}$ of $g$ with support in $W_{\omega}$ and $W_{\alpha}$ respectively, two points $z_{\omega}$ and $z_{\alpha}$ in $U_{z_{0}}$, and two iterates $g_{\omega}^{n_{\omega}}\left(z_{\omega}\right), g_{\alpha}^{-n_{\alpha}}\left(z_{\alpha}\right)$. The two perturbations $g_{\alpha}, g_{\omega} \in \mathcal{U}$ have disjoint supports $W_{\alpha}$ and $W_{\omega}$, hence one can introduce the composed perturbation $\hat{g} \in \mathcal{U}$. Since the forward orbit of $z_{\omega}$ by $g_{\omega}$ is contained in $\hat{W}_{\omega}$, it does not intersect $W_{\alpha}$ and for the same reason, the backward orbit of $z_{\alpha}$ by $g_{\alpha}$ does not intersect $U_{\omega}$. Hence, these two half orbits remain unchanged by $\hat{g}$. Moreover, there are two iterates $\hat{g}^{n_{\omega}}\left(z_{\omega}\right)$ and $\hat{g}^{-n_{\alpha}}\left(z_{\alpha}\right)$ with $n_{\alpha}, n_{\omega}<N$ in $W_{\omega}$ and $W_{\alpha}$ respectively such that the forward orbit of $\hat{g}^{n_{\omega}}\left(z_{\omega}\right)$ and the backward orbit of $\hat{g}^{-n_{\alpha}}\left(z_{\alpha}\right)$ by $\hat{g}$ are $\eta_{0} / 2$-close to $\mathcal{X}_{\omega}$ and $\mathcal{X}_{\alpha}$ respectively.

The connecting lemma allows to perturb $\hat{g}$ in $U_{z_{0}}$ and its $N$ first forward iterates in order to connect $\hat{g}^{-n_{\alpha}}\left(z_{\alpha}\right)$ to $\hat{g}^{n_{\omega}}\left(z_{\omega}\right)$ in an orbit which intersects $U_{z_{0}}$ at some point $z$. The new diffeomorphism $g$ belongs to $\mathcal{U}$ since all the perturbations have disjoint supports. 
The $\eta_{0} / 2$-neighborhood of the orbit of $z$ by $g$ contains the orbit of $z_{0}$ by $f$. Moreover, the forward orbit of $\hat{g}^{n_{\omega}}\left(z_{\omega}\right)$ by $g_{\omega}$ is the same as by $g$ and the backward orbit of $\hat{g}^{-n_{\alpha}}\left(z_{\alpha}\right)$ by $g_{\alpha}$ the same as by $g$. Hence, the $\eta_{0} / 2$-neighborhood of the orbit of $z$ by $g$ contains the sets $\mathcal{X}_{\alpha}$ and $\mathcal{X}_{\omega}$. Consequently (using item 2)), the $\eta_{0}$-neighborhood of $\mathcal{O}(z)$ contains $\mathcal{X}$. By construction, the orbit of $z$ by $g$ is contained in $W$, the $\eta_{0}$-neighborhood of $\mathcal{X}$. We thus proved that the closure of the orbit of $z$ by $g$ and $\mathcal{X}$ are $\eta_{0}$-close in the Hausdorff topology.

5.3.2. Proof of proposition 21. - Note that if $\mathcal{X}_{\omega}$ supports a minimal dynamics, then, one can apply proposition 10. One immediately gets a point $z_{\omega} \in U_{z_{0}}$ and a perturbation $g_{\omega} \in \mathcal{U}$ with support in $W_{\omega}$ such that the forward orbit of $z_{\omega}$ is contained in $\hat{W}_{\omega}$ and accumulates on a part of $\mathcal{X}_{\omega}$. Since $\mathcal{X}_{\omega}$ is minimal, it accumulates on the whole set $\mathcal{X}_{\omega}$. Hence, one gets the conclusion of proposition 21 in this case. We will now assume that $\mathcal{X}_{\omega}$ is not minimal.

By shrinking $\mathcal{U}$, one will assume that it satisfies condition $(\mathrm{F})$. The connecting lemma associates to $(\mathcal{U}, f)$ an integer $N \geq 1$. One can shrink the open set $W_{\omega}$ so that it is contained in the $\eta_{0}$-neighborhood of $\mathcal{X}_{\omega}$. We need some preliminary constructions, that are described in the next two lemmas.

Lemma 7. - There exist

- a finite set $\left\{p_{1}, \ldots, p_{r-1}\right\}$ in $\mathcal{X}_{\omega}$, with $r \geq 2$, and a point $p_{r} \in(\mathcal{X} \backslash$ $\left.\mathcal{X}_{\omega}\right) \cap W_{\omega}$,

- two compact sets $K_{\omega} \subset \mathcal{X} \cap W_{\omega}$ and $\hat{K}_{\omega} \subset \mathcal{X} \cap \hat{W}_{\omega}$,

- an invariant compact subset $A \subset \mathcal{X}_{\omega}$ that supports a minimal dynamics,

- a small neighborhood $\hat{W}_{A}$ of $A$, contained in $W_{\omega}$,

such that:

1. The points $f^{j}\left(p_{k}\right)$ for $k \in\{1, \ldots, r\}$ and $j \in\{-N, \ldots, N\}$ are pairwise distinct.

2. For any $k, \ell \in\{1, \ldots, r\}$, with $k<\ell$, we have $p_{\ell} \prec_{K_{\omega}} p_{k}$.

3. We have $z_{0} \prec_{\hat{K}_{\omega}} p_{r}$.

4. We have $p_{1} \prec_{\hat{W}_{A}} A$.

5. The points $f^{j}\left(p_{k}\right)$ for $k \in\{2, \ldots, r\}$ and $j \in\{-N, \ldots, N\}$ are not in $\hat{W}_{A}$.

6. The union of $A$ with the closure of the orbits of the points $p_{k}$ is $\eta_{0} / 2$-close to $\mathcal{X}_{\omega}$ for the Hausdorff distance.

Proof. - By proposition 6 and the remark 1.6.2 which follows the proposition, there exists an invariant compact set $A \subset \mathcal{X}_{\omega}$ which supports 
a minimal dynamics and such that $x \prec \mathcal{X}_{\omega} y$ for any $x \in \mathcal{X}_{\omega}$ and $y \in A$. Since $\mathcal{X}_{\omega}$ is not minimal, we have $A \neq \mathcal{X}_{\omega}$. As in proposition 5, there exists a finite subset $\left\{p_{2}, \ldots, p_{r-1}\right\} \subset \mathcal{X}_{\omega} \backslash A$ of points that are non-periodic, have distinct orbits and such that the union of $A$ with the orbits of the points $p_{2}, \ldots, p_{r-1}$ has a closure which is $\eta_{0} / 2$-close to $\mathcal{X}_{\omega}$ for the Hausdorff distance. Since $\mathcal{X}_{\omega}$ is a weak orbit whose relation $\prec_{\mathcal{X}_{\omega}}$ is transitive, one may order the points such that $p_{\ell} \prec \mathcal{X}_{\omega} p_{k}$ when $k<\ell$.

We also have $z_{0} \prec_{\hat{K}_{\omega}} p_{r-1}$. As in lemma 6, one deduces that there exists a point $p_{r} \in\left(\mathcal{X} \backslash \mathcal{X}_{\omega}\right)^{n} \cap W_{\omega}$ such that $z_{0} \prec_{\hat{K}_{\omega}} p_{r}$, and such that $p_{r} \prec_{K_{\omega}}$ $p_{r-1}$ where $K_{\omega}$ is a compact set contained in $W_{\omega} \cap \mathcal{X}$ that contains $\mathcal{X}_{\omega}$ (for instance, $K_{\omega}$ can be the intersection of $\mathcal{X}$ with any compact neighborhood of $\mathcal{X}_{\omega}$ ). If $p_{r}$ has been chosen close enough to $\mathcal{X}_{\omega}$, it is not a periodic point with period less than or equal to $2 N$, by condition (A).

Let $\hat{W}_{A}$ be a neighborhood of $A$, contained in $W_{\omega}$ and that is disjoint from the points the points $f^{j}\left(p_{k}\right)$ for $k \in\{2, \ldots, r\}$ and $j \in\{-N, \ldots, N\}$. We have $p_{2} \prec_{c X_{\omega}} A$ by definition of $A$. By lemma 6, there exists a point $p_{1}$ in $\hat{W}_{A}$ such that $p_{2} \prec_{\mathcal{X}_{\omega}} p_{1}$ and $p_{1} \prec_{\hat{W}_{A}} A$. Moreover, if the point $p_{1}$ is chosen close enough to $A$, all the points $f^{j}\left(p_{k}\right)$ with $k \in\{1, \ldots, r\}$ and $j \in\{-N, \ldots, N\}$ are pairwise distinct.

Lemma 8. - There exist a constant $\varepsilon>0$ and two neighborhoods $V_{r} \subset$ $U_{r}$ of $p_{r}$ in $B\left(p_{r}, \varepsilon\right)$, such that:

1. The balls $f^{j}\left(B\left(p_{k}, \varepsilon\right)\right)$ for $k \in\{1, \ldots, r\}$ and $j \in\{-N, \ldots, N\}$ are pairwise disjoint, disjoint from $A$, and, when $k>1$, disjoint from $\hat{W}_{A}$.

2. The closing lemma may be applied to $(\mathcal{U}, f)$ in the neighborhoods $\left(V_{r}, U_{r}\right)$.

3. Let $g$ be any perturbation of $f$ with support in the balls $f^{j}\left(B\left(p_{k}, \varepsilon\right)\right)$ (with $k \in\{1, \ldots, r\}$ and $j \in\{-N, \ldots, N\}$ ) and in the $\varepsilon$-neighborhood of $A$. Then, the $\eta_{0}$-neighborhood of any segment of orbit by $g$ that intersects all the balls $B\left(p_{k}, \varepsilon\right)$ and the $\varepsilon$-neighborhood of $A$ contains $\mathcal{X}_{\omega}$.

4. In the $\varepsilon$-neighborhood of $\mathcal{X}$, there is no segment of orbit $\left(z, f(z), \ldots, f^{n}(z)\right)$ such that $z$ belongs to some ball $B\left(p_{k}, \varepsilon\right)$, with $k \in\{1, \ldots, r-1\}$ and $f^{n}(z)$ to the ball $B\left(p_{r}, \varepsilon\right)$.

Proof. - Let $\varepsilon>0$ be a small constant. By items 1) and 5) of lemma 7 , for $\varepsilon$ small enough, the item 1) of the lemma is satisfied. Moreover, let $g$ be a perturbation of $f$ with support in the balls $f^{j}\left(B\left(p_{k}, \varepsilon\right)\right)$ and in the $\varepsilon$-neighborhood of $A$. Since $\varepsilon$ is small, any segment of orbit of $g$ that inter- 
sects all the balls $B\left(p_{k}, \varepsilon\right)$ and the $\varepsilon$-neighborhood of $A$ contains in its $\eta_{0} / 2$ neighborhood the orbits of the points $p_{k}$, with $k \in\{1, \ldots, r-1\}$ by $f$. Since the dynamics of $f$ on $A$ is minimal, it contains also in its $\eta_{0} / 2$-neighborhood the set $A$. By item 6) of lemma 7, one deduces that the $\eta_{0}$-neighborhood of this segment of orbit contains $\mathcal{X}_{\omega}$. One gets item 3 ).

The item 4) is verified for $\varepsilon$ small enough, otherwise one would have $p_{k} \prec \mathcal{X} p_{r}$ for some $k \in\{1, \ldots, r-1\}$ but since the points $p_{1}, \ldots, p_{r-1}$ belong to $\mathcal{X}_{\omega}$ and $p_{r}$ to $\mathcal{X} \backslash \mathcal{X}_{\omega}$, this would contradict the assumption $\left.{ }^{*}\right)$ of proposition 21. It remains to apply Hayashi's connecting lemma (theorem 5) at $p_{r}$ in order to build the neighborhoods $V_{r}, U_{r}$, contained in $B\left(p_{r}, \varepsilon\right)$.

We now finish to prove proposition 21 (and theorem 21).

Proof (End of the proof of proposition 21). - Let $W$ be the $\varepsilon$-neighborhood of $\mathcal{X}$. By item 3) of lemma [7, there exists a finite segment of orbit $Z_{a}=$ $\left(z_{\omega}, f\left(z_{\omega}\right), \ldots, f^{n_{a}}\left(z_{\omega}\right)\right)$ which is contained in $W \cap \hat{W}_{\omega}$ and such that $z_{\omega}$ belongs to $U_{z_{0}}$ and $f^{n_{a}}\left(z_{\omega}\right)$ to $V_{r}$. By item 4) of lemma 8, $Z_{a}$ does not intersect the balls $B\left(p_{k}, \varepsilon\right)$ for $k \in\{1, \ldots, r-1\}$.

Let $\eta>0$ be a constant smaller than $\varepsilon$ and such that $B\left(p_{r}, \eta\right)$ is contained in $V_{r}$. By items 1) and 2) of lemma 7, one can apply proposition 9 to $f^{-1}$ for the set $\left\{p_{1}, \ldots, p_{r}\right\}$ and the open set $W \cap W_{\omega}$ : there is a perturbation $g_{b} \in \mathcal{U}$ of $f$ with support in

$$
\bigcup_{1 \leq k \leq r} \bigcup_{1 \leq j \leq N} f^{-j}\left(\mathrm{~B}\left(p_{k}, \eta\right)\right)
$$

and some integer $n_{b} \geq 1$ such that the finite segment of orbit $Z_{b}=\left(g_{b}^{-n_{b}}\left(p_{1}\right), g_{b}^{-n_{b}+1}\left(p_{1}\right), \ldots, p_{1}\right.$ is contained in $W \cap W_{\omega}$ and crosses all the balls $\mathrm{B}\left(p_{k}, \eta\right)$.

Let us denote by $m_{b} \in\left\{0, \ldots, n_{b}\right\}$ the smallest integer such that $g_{b}^{-m_{b}}\left(p_{1}\right)$ belongs to $\mathrm{B}\left(p_{r}, \varepsilon\right)$. The iterates $\left(g_{b}^{-n_{b}}\left(p_{1}\right), \ldots, g_{b}^{-m_{b}}\left(p_{1}\right)\right)$ can not intersect the other balls $\mathrm{B}\left(p_{k}, \varepsilon\right)$ with $k \in\{1, \ldots, r-1\}$ by item 4$)$ of lemma 8 . Hence, the orbit $\left(g_{b}^{-m_{b}}\left(p_{1}\right), \ldots, p_{1}\right)$ crosses all the balls $\mathrm{B}\left(p_{k}, \varepsilon\right)$ with $k \in\{1, \ldots, r-1\}$. By remark 5.1 after the proof of proposition 9, we deduce that it was not necessary to perturb $f$ close to the point $p_{r}$ and its $N$ first backward iterates in order to build the orbit $Z_{b}$ of $g_{b}$. Thus, one can assume that the support of the perturbation $g_{b}$ is contained in the union of the balls $B\left(p_{k}, \varepsilon\right)$ with $k \in\{0, \ldots, r-1\}$ and their $N$ first backward iterates. By item 1) of lemma 8, this support is disjoint from $U_{r}$ and its $N$ backward iterates.

One chooses now at $f\left(p_{1}\right)$ a small neighborhood $U \subset \mathrm{B}\left(f\left(p_{1}\right), \varepsilon\right)$ whose $N$ first forward iterates by $f$ are disjoint from $A$ and from the segments of orbit $Z_{a}$ by $f$ and $Z_{b}$ by $g_{b}$. One considers a smaller neighborhood $V \subset U$ 
of $f\left(p_{1}\right)$ such that the connecting lemma can be applied for $(f, \mathcal{U})$ in $V \subset U$ with the time $N$.

Let us introduce a neighborhood $W_{A} \subset \hat{W}_{A}$ of $A$ which is contained in the $\varepsilon$-neighborhood of $A$ and which is disjoint from the sequences $Z_{a}$ and $Z_{b}$, from the set $U$ and its $N$ first forward iterates and (by item 1) of lemma 8) from the balls $f^{-j}\left(B\left(p_{k}, \varepsilon\right)\right)$ with $j \in\{0, \ldots, N\}$ and $k \in\{1, \ldots, r\}$. By proposition 10 and item 4) of lemma 7 , there exists a perturbation $g_{c} \in \mathcal{U}$ of $f$ in $\mathcal{U}$ whose support is contained in $W_{A} \backslash A$ and a point $p_{0}$ in $f^{N}(V)$ whose forward orbit $Z_{c}=\left\{g_{c}^{n}\left(p_{0}\right), n \geq 0\right\}$ by $g_{c}$ is contained in $\hat{W}_{A}$ and accumulates on a subset of $A$. Moreover, $Z_{c}$ is disjoint from the support of $g_{b}$ by item 1) of lemma 8 .

The perturbation $g_{b}$ and $g_{c}$ have disjoint supports, so that the composed perturbation $\hat{g}$ belongs to $\mathcal{U}$. By construction, the sequences $Z_{a}, Z_{b}$ and $Z_{c}$ remain segments of orbits of $\hat{g}$. One can now apply the connecting lemma a first time to $\hat{g}$ in the sets $U, V$ between the points $\hat{g}^{-n_{b}}\left(p_{1}\right)$ and $\hat{g}^{N+1}\left(p_{0}\right)$ and then a second time to $\hat{g}^{-1}$ in the sets $V_{r}, U_{r}$ between the points $z_{\omega}$ and $p_{1}$ (by item 2) of lemma 8). The supports of these two perturbation are disjoint and disjoint from the support of the perturbation $\hat{g}$ of $f$. Hence, by composition one obtains a diffeomorphism $g_{\omega}$ in $\mathcal{U}$.

Note that the segments of orbit $\left(g_{b}^{-m_{b}}\left(p_{1}\right), \ldots, p_{1}\right)$ by $\hat{g}$ was not modified by these last perturbations. Moreover, it now belongs to the forward orbit of $z_{\omega}$. In particular, the point $g_{b}^{-m_{b}}\left(p_{1}\right)$ is now an iterate $g_{\omega}^{n_{\omega}}\left(z_{\omega}\right)$ of $z_{\omega}$. This also shows that the forward orbit of $z_{\omega}$ by $g_{\omega}$ intersects all the balls $B\left(p_{k}, \varepsilon\right)$. By construction, the forward orbit of $z_{\omega}$ by $g_{\omega}$ coincides after a large iterate with the forward orbit of an iterate of $Z_{c}$; hence, the forward orbit of $z_{\omega}$ by $g_{\omega}$ intersects the $\varepsilon$-neighborhood of $A$. By lemma 8 , the $\varepsilon$-neighborhood of this half orbit contains the set $\mathcal{X}_{\omega}$. On the other hand, by our constructions, the forward orbit of $z_{\omega}$ by $g_{\omega}$ is contained in $\hat{W}_{\omega}$ and the forward orbit of $g_{\omega}^{n_{\omega}}\left(z_{\omega}\right)$ is contained in $W_{\omega}$ (which is contained in the $\eta_{0}$-neighborhood of $\left.\mathcal{X}_{\omega}\right)$. Hence, the closure of the forward orbit of $g_{\omega}^{n_{\omega}}\left(z_{\omega}\right)$ by $g_{\omega}$ is $\eta_{0}$-close to $\mathcal{X}_{\omega}$ for the Hausdorff distance. This concludes the proof of proposition 21 .

\section{REFERENCES}

AbBC. F. Abdenur, C. Bonatti and S. Crovisier, Global dominated splittings and the $C^{1}$ Newhouse phenomenon, Proc. Amer. Math. Soc., 134, (2006), 2229-2237.

ABCD. F. Abdenur, C. Bonatti, S. Crovisier and L. DíAz, Generic diffeomorphisms on compact surfaces, Fund. Math., 187, (2005), 127-159.

AD. F. AbDenur and L. DíAz, Pseudo-orbit shadowing in the $C^{1}$-topology, to appear in Discrete Cont. Dyn. Syst.

AS. R. Abraham and S. Smale, Nongenericity of $\Omega$-stability, Global analysis I, Proc. Symp. Pure Math. AMS, 14, (1970), 5-8. 
A . M.-C. Arnaud, Création de connexions en topologie $C^{1}$, Ergod. Th. E Dynam. Sys., 21, (2001), 339-381.

$\mathrm{A}_{2}$. M.-C. Arnaud, Approximation des ensembles $\omega$-limites des difféomorphismes par des orbites périodiques, Ann. Sci. École Norm. Sup., 36, (2003), 173-190.

ArBC. M.-C. Arnaud, C. Bonatti and S. Crovisier, Dynamiques symplectiques génériques, Ergod. Th. EG Dynam. Sys., 25, (2005), 1401-1436.

BC. C. Bonatti and S. Crovisier, Récurrence et généricité, Invent. Math., 158, (2004), 33-104.

$\mathrm{BD}_{1}$. C. Bonatti and L. DíAz, Persistent nonhyperbolic transitive diffeomorphisms, Ann. of Math., 143, (1996), 357-396.

$\mathrm{BD}_{2}$. C. Bonatti and L. DíAz, On maximal transitive sets of generic diffeomorphisms, Publ. Math. Inst. Hautes Études Sci., 96, (2003), 171-197.

BDP. C. Bonatti, L. DíAz and E. Pujals, A $C^{1}$-generic dichotomy for diffeomorphisms: weak forms of hyperbolicicity or infinitely many sinks or sources, Ann. Math., 158, (2003), 355-418.

BDT. C. Bonatti, L. DíAz and G. Turcat, Pas de "shadowing lemma" pour des dynamiques partiellement hyperboliques, C. R. Acad. Sci. Paris, 330, (2000), 587-592.

B. R. BOWEn, Equilibrium states and the ergodic theory of Anosov diffeomorphisms, Lecture Notes in Mathematics, 470, Springer-Verlag, Berlin-New York (1975).

C. C. Conley, Isolated invariant sets and Morse index, CBMS Regional Conference Series in Mathematics, 38, AMS Providence (1978).

CMP. C. Carballo, C. Morales and M.-J. PaCífico, Homoclinic classes for $C^{1}$-generic vector fields, Ergodic Th. ES Dynam. Sys., 23, (2003), 1-13.

CP. R. Corless and S. Pilyugin, Approximate and real trajectories for generic dynamical systems, J. Math. Anal. Appl., 189, (1995), 409-423.

D. W. De melo, Structural stability of diffeomorphisms on two-manifolds, Invent. Math., 21, (1973), 233246.

GW. G. GAN and L. WEN, Heteroclinic cycles and homoclinic closures for generic diffeomorphisms, J. Dynam. Differential Equations, 15, (2003), 451-471.

GST. S. Gonchenko, L. Shilńikov, D. Turaev, Dynamical phenomena in systems with structurally unstable Poincaré homoclinic orbits, Chaos, 6, (1996), 15-31.

H. S. HAYASHI, Connecting invariant manifolds and the solution of the $C^{1}$-stability and $\Omega$-stability conjectures for flows, Ann. of Math., 145, (1997), 81-137 and 150 (1999), 353-356.

K. I. KupkA, Contribution à la théorie des champs génériques, Contributions to Differential Equations, 2, (1963), 457-484 and 3, (1964), 411-420.

$\mathrm{M}_{1}$. R. MAÑé, Contributions to the stability conjecture, Topology, 17, (1978), 383-396.

$\mathrm{M}_{2}$. R. MAÑ́́, An ergodic closing lemma, Ann. of Math., 116, (1982), 503-540.

M3. R. MAÑÉ, A proof of the $C^{1}$ stability conjecture, Publ. Math. Inst. Hautes Études Sci., 66, (1988), $161-210$

Maz. M. MAZur, Tolerance stability conjecture revisited, Topology Appl., 131, (2003), 33-38.

$\mathrm{N}_{1}$. S. Newhouse, Hyperbolic limit sets, Trans. AMS, 167, (1972), 125-150.

$\mathrm{N}_{2}$. S. Newhouse, Diffeomorphisms with infinitely many sinks, Topology, 13, (1974), 9-18.

$\mathrm{N}_{3}$. S. Newhouse, The abundance of wild hyperbolic sets and nonsmooth stable sets for diffeomorphisms, Inst. Hautes tudes Sci. Publ. Math., 50, (1979), 101-151.

O. K. OdAni, Generic homeomorphisms have the pseudo-orbit tracing property, Proc. Amer. Math. Soc., 110, (1990), 281-284.

P. J. PAlis, On the $C^{1} \Omega$-stability conjecture, Publ. Math. Inst. Hautes Études Sci., 66, (1988), 211-215.

PS. J. PAlis and S. Smale, Structural stability theorem, Proc. AMS Symp. Pure Math., 14, (1970), $223-232$.

PT. J. PALIS and F. TAKEns, Hyperbolicity \& sensitive chaotic dynamics at homoclinic bifurcations, Cambridge studies in advanced mathematics, 35, Cambridge University Press (1993).

PV. J. PALIS and M. VIANA, High dimension diffeomorphisms displaying infinitely many periodic attractors, Ann. of Math., 140, (1994), 207-250.

Pi. S. Pilyugin, Shadowing in dynamical systems, Lecture Notes in Mathematic, 1706, (1999).

$\mathrm{Pu}_{1}$. C. Pugh, The closing lemma, Amer. J. Math., 89, (1967), 956-1009. 
$\mathrm{Pu}_{2}$. C. Pugh, An improved closing lemma and a general density theorem, Amer. J. Math., 89, (1967), 1010-1021

PR. C. Pugh, and C. Robinson, The $C^{1}$-closing lemma, including hamiltonians, Ergod. Th. E Dynam. Sys., 3, (1983), 261-314.

Rob. J. Robbin, A structural stability theorem, Ann. of Math., 94, (1971), 447-493.

Rob $_{1}$. C. Robinson, Generic properties of conservative systems, Amer. J. Math., 92, (1970), 562-603 and 897-906.

Rob $_{2}$. C. Robinson, $C^{r}$-structural stability implies Kupka-Smale, Dynamical systems, (Proc. Sympos., Univ. Bahia, Salvador, 1971), 443-449, Academic Press, New York (1973).

Rob $_{3}$. C. Robinson Structural stability of $C^{1}$-diffeomorphisms, J. Diff. Equ., 22, (1976), 28-73.

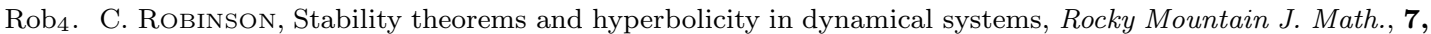
(1977), 425-437.

Rom. N. Romero, Persistence of homoclinic tangencies in higher dimensions, Ergod. Th. E Dynam. Sys., 15, (1995), 735-757.

Sa. K. SAKAI, Diffeomorphisms with weak shadowing, Fund. Math., 168, (2001), 57-75.

$\mathrm{Sh}_{1}$. M. Shub, Stability and genericity for diffeomorphisms, Dynamical systems, (Proc. Sympos., Univ. Bahia, Salvador, 1971), 493-514, Academic Press, New York (1973).

$\mathrm{Sh}_{2}$. M. Shub, Topologically transitive diffeomorphisms of $T^{4}$, Lecture Notes in Mathematics, 206, (1971), 39-40.

Si. C. Simon, A 3-dimensional Abraham-Smale example, Proc. Amer. Math. Soc., 34, (1972), 629-630.

$\mathrm{Sm}_{1}$. S. Smale, Stable manifolds for differential equations and diffeomorphisms, Ann. Scuola Norm. Sup. Pisa, 17, (1963), 97-116.

$\mathrm{Sm}_{2}$. S. Smale, Differentiable dynamical systems, Bull. Amer. Math. Soc., 73, (1967), $747-817$.

T1. F. TAKens, On Zeeman's tolerance stability conjecture, Lecture Notes in Math., 197, (1971), $209-219$.

$\mathrm{T}_{2}$. $\quad$ F. TAkens, Tolerance stability, Lecture Notes in Math., 468, (1975), 293-304.

We. $\quad$ L. Wen, A uniform $C^{1}$ connecting lemma, Discrete Contin. Dyn. Syst., 8, (2002), 257-265.

WX. L. WEn and Z. XIA, $C^{1}$ connecting lemmas, Trans. Amer. Math. Soc., 352, (2000), 5213-5230.

Wh. W. White, On the tolerance stability conjecture, Dynamical systems, (Proc. Sympos., Univ. Bahia, Salvador, 1971), 663-665, Academic Press, New York (1973).

YY. G. YAU and J. Yorke, An open set of maps for which every point is absolutely non-shadowable, Proc. Amer. Math. Soc., 128, (2000), 909-918.

S. C.

CNRS - Laboratoire Analyse, Géométrie et Applications, UMR 7539, Institut Galilée, Université Paris 13, Avenue J.-B. Clément, 93430 Villetaneuse, France crovisie@math.univ-paris13.fr 